JOURNAL OF THE

AMERICAN MATHEMATICAL SOCIETY

Volume 22, Number 4, October 2009, Pages 1099-1156

S 0894-0347(09)00635-3

Article electronically published on May 6, 2009

\title{
RIGOROUS DERIVATION OF THE GROSS-PITAEVSKII EQUATION WITH A LARGE INTERACTION POTENTIAL
}

\author{
LÁSZLÓ ERDŐS, BENJAMIN SCHLEIN, AND HORNG-TZER YAU
}

\section{INTRODUCTION}

We consider a bosonic system of $N$ particles with a repulsive interaction. The states of the system are given by elements of the Hilbert space $L_{s}^{2}\left(\mathbb{R}^{3 N}\right)$, the subspace of $L^{2}\left(\mathbb{R}^{3 N}\right)$ consisting of permutation symmetric wave functions. We are interested in describing the time evolution of special initial wave functions $\psi_{N} \in L_{s}^{2}\left(\mathbb{R}^{3 N}\right)$ that exhibit complete Bose-Einstein condensation.

For a given wave function $\psi_{N}$, we define the density matrix $\gamma_{N}=\left|\psi_{N}\right\rangle\left\langle\psi_{N}\right|$ associated with $\psi_{N}$ as the orthogonal projection onto $\psi_{N}$. Moreover, for $k=$ $1, \ldots, N$, we define the $k$-particle marginal density $\gamma_{N}^{(k)}$, associated with $\psi_{N}$, by taking the partial trace of $\gamma_{N}$ over the last $(N-k)$ variables. In other words, $\gamma_{N}^{(k)}$ is defined as a positive trace-class operator on $L^{2}\left(\mathbb{R}^{3 k}\right)$ with kernel given by

$$
\gamma_{N}^{(k)}\left(\mathbf{x}_{k} ; \mathbf{x}_{k}^{\prime}\right)=\int \mathrm{d} \mathbf{x}_{N-k} \psi_{N}\left(\mathbf{x}_{k}, \mathbf{x}_{N-k}\right) \bar{\psi}_{N}\left(\mathbf{x}_{k}^{\prime}, \mathbf{x}_{N-k}\right) .
$$

Here and in the following we use the notation $\mathbf{x}_{k}=\left(x_{1}, \ldots, x_{k}\right), \mathbf{x}_{k}^{\prime}=\left(x_{1}^{\prime}, \ldots, x_{k}^{\prime}\right)$, and $\mathbf{x}_{N-k}=\left(x_{k+1}, \ldots, x_{N}\right)$; we will also denote $\mathbf{x}=\left(x_{1}, x_{2}, \ldots, x_{N}\right)$. A sequence $\left\{\psi_{N}\right\}_{N \in \mathbb{N}}$, with $\psi_{N} \in L_{s}^{2}\left(\mathbb{R}^{3 N}\right)$ for all $N$, is said to exhibit complete Bose-Einstein condensation in $\varphi \in L^{2}\left(\mathbb{R}^{3}\right)$ if

$$
\gamma_{N}^{(1)} \rightarrow|\varphi\rangle\langle\varphi| \quad \text { as } N \rightarrow \infty
$$

in the trace-norm topology (here and in the following $|\varphi\rangle\langle\varphi|$ indicates the orthogonal projection onto $\varphi$ ). Physically, complete Bose-Einstein condensation means that all particles in the system, apart from a fraction vanishing as $N \rightarrow \infty$, are described by the same one-particle wave function $\varphi$, known as the condensate wave function. Note that (1.1) implies that

$$
\gamma_{N}^{(k)} \rightarrow|\varphi\rangle\left\langle\left.\varphi\right|^{\otimes k}\right.
$$

for all $k \geq 1$, as was first proven by Lieb and Seiringer in [18.

Received by the editors April 15, 2008.

2000 Mathematics Subject Classification. Primary 82C10, 35Q55.

The first author was partially supported by SFB/TR12 Project from DFG.

The second author was supported by a Kovalevskaja Award from the Humboldt Foundation.

The third author was partially supported by NSF grants DMS-0602038, 0757425, and 0804279. 
The time-evolution of $N$ boson systems is governed by the Schrödinger equation

$$
i \partial_{t} \psi_{N, t}=H_{N} \psi_{N, t}
$$

with the Hamiltonian operator

$$
H_{N}=\sum_{j=1}^{N}-\Delta_{j}+\sum_{i<j} V_{N}\left(x_{i}-x_{j}\right) .
$$

Here and in the following we will use the convention $\Delta_{j}=\Delta_{x_{j}}$ and $\nabla_{j}=\nabla_{x_{j}}$. We consider the scaling introduced by Lieb, Seiringer and Yngvason in 20 for the interaction potential $V_{N}$, i.e. we fix a nonnegative potential $V$ and then rescale it by defining $V_{N}(x)=N^{2} V(N x)$. This scaling is chosen so that the scattering length of $V_{N}$ is of the order $1 / N$.

We recall that the scattering length associated with a potential $V$ decaying sufficiently fast at infinity ( $V$ has to be integrable at infinity) is defined through the solution of the zero-energy scattering equation

$$
\left(-\Delta+\frac{1}{2} V\right) f=0
$$

with boundary condition $f(x) \rightarrow 1$ as $|x| \rightarrow \infty$. We usually write $f=1-w$. The scattering length of $V$, which is a measure of the effective range of the interaction, is defined by

$$
a_{0}=\lim _{|x| \rightarrow \infty}|x| w(x) .
$$

An equivalent definition of the scattering length is given by the formula

$$
\int \mathrm{d} x V(x)(1-w(x))=8 \pi a_{0} .
$$

By these definitions, it is clear that if $a_{0}$ denotes the scattering length of the potential $V$, then the scattering length of the scaled potential $V_{N}$ is given by $a=a_{0} / N$.

Our main result is as follows. Suppose that the family of wave functions $\left\{\psi_{N}\right\}_{N \in \mathbb{N}}$ exhibits complete Bose-Einstein condensation (1.1) with some $\varphi \in H^{1}\left(\mathbb{R}^{3}\right)$ and assume its energy per particle to be bounded (in the sense that $\left\langle\psi_{N}, H_{N} \psi_{N}\right\rangle \leq C N$ for all $N$ ). Denote by $\psi_{N, t}$ the solution of the Schrödinger equation (1.3) with initial data $\psi_{N, 0}=\psi_{N}$. Under appropriate conditions on the potential $V$, we show that, for every time $t \in \mathbb{R}$, the family $\left\{\psi_{N, t}\right\}_{N \in \mathbb{N}}$ still exhibits complete condensation and that the condensate wave function evolves according to a cubic nonlinear Schrödinger equation known as the Gross-Pitaevskii equation. In other words, if $\gamma_{N, t}^{(1)}$ denotes the one-particle marginal density associated with $\psi_{N, t}$, we prove that

$$
\gamma_{N, t}^{(1)} \rightarrow\left|\varphi_{t}\right\rangle\left\langle\varphi_{t}\right|
$$

as $N \rightarrow \infty$, where $\varphi_{t}$ is determined by the nonlinear Gross-Pitaevskii equation

$$
i \partial_{t} \varphi_{t}=-\Delta \varphi_{t}+8 \pi a_{0}\left|\varphi_{t}\right|^{2} \varphi_{t}
$$

with initial data $\varphi_{0}=\varphi$. The cubic nonlinear term expresses the local on-site self-interaction of the condensate wave function. Due to the strongly localized interaction, the many-body wave function develops a singular correlation structure on the scale $1 / N$. As $N$ goes to infinity, this short scale structure produces the scattering length as a coupling constant in the limiting Gross-Pitaevskii equation. 
This result gives a mathematical description of the dynamics of initial data exhibiting complete Bose-Einstein condensation. The simplest example of such initial data are product states $\psi_{N}=\varphi^{\otimes N}$ for arbitrary $\varphi \in L^{2}\left(\mathbb{R}^{3}\right)$. Physically more interesting examples of complete Bose-Einstein condensates are the ground states of trapped Bose gases. A trapped Bose gas in the Gross-Pitaevskii scaling is an $N$-boson system described by the Hamiltonian

$$
H_{N}^{\mathrm{trap}}=\sum_{j=1}^{N}\left(-\Delta_{x_{j}}+V_{\mathrm{ext}}\left(x_{j}\right)\right)+\sum_{i<j}^{N} V_{N}\left(x_{i}-x_{j}\right),
$$

where $V_{\text {ext }}(x) \geq 0$ with $V_{\text {ext }}(x) \rightarrow \infty$ as $|x| \rightarrow \infty$ is a confining potential. The Hamiltonian (1.9) therefore describes a system of $N$ particles confined by the external potential $V_{\text {ext }}$ into a volume of order one, interacting through a potential with effective range of the order $1 / N$. Since the typical distance between neighboring particles is of order $N^{-1 / 3}$, and thus much bigger than the effective range of the interaction, (1.9) describes a very dilute system.

In [20, Lieb, Seiringer and Yngvason studied the ground state energy $E_{N}$ of the Hamiltonian (1.9) with a spherically symmetric interaction, $V(x)=V(|x|)$, and they proved that

$$
\lim _{N \rightarrow \infty} \frac{E_{N}}{N}=\min _{\varphi \in L^{2}\left(\mathbb{R}^{3}\right)} \mathcal{E}_{\mathrm{GP}}(\varphi)
$$

where $\mathcal{E}_{\mathrm{GP}}$ denotes the so-called Gross-Pitaevskii energy functional

$$
\mathcal{E}_{\mathrm{GP}}(\varphi)=\int \mathrm{d} x\left(|\nabla \varphi|^{2}+V_{\text {ext }}(x)|\varphi(x)|^{2}+4 \pi a_{0}|\varphi(x)|^{4}\right)
$$

In [18, Lieb and Seiringer then proved that the ground state of (1.9) exhibits complete Bose-Einstein condensation in the minimizer $\phi_{G P}$ of the Gross-Pitaevskii energy functional $\mathcal{E}_{\mathrm{GP}}$. In other words, they proved that if $\psi_{N}^{\text {trap }}$ denotes the ground state vector of (1.9), then the corresponding one-particle marginal density $\gamma_{N \text {,trap }}^{(1)}$ satisfies

$$
\gamma_{N, \text { trap }}^{(1)} \rightarrow\left|\phi_{\mathrm{GP}}\right\rangle\left\langle\phi_{\mathrm{GP}}\right|
$$

as $N \rightarrow \infty$, in the trace-norm topology. A survey of results concerning the ground state properties of the dilute bosonic gases can be found in [19.

Since $\psi_{N}^{\text {trap }}$ describes a complete Bose-Einstein condensate, we can apply (1.7) to study its time evolution with respect to the Hamiltonian (1.4); it follows that, for every fixed $t \in \mathbb{R}, e^{-i H_{N} t} \psi_{N}^{\text {trap }}$ exhibits complete Bose-Einstein condensation in the one-particle state described by the solution $\varphi_{t}$ of the Gross-Pitaevskii equation (1.8) with initial data $\phi_{\mathrm{GP}}$. This result gives the mathematical description of recent experiments (initiated in [7, 3]) where the dynamics of Bose-Einstein condensates have been observed.

We already proved (1.7) in 12] (some partial results were previously obtained in [10]) under the assumption of a sufficiently weak potential $V$. More precisely, in 
[12] we required the dimensionless parameter

$$
\rho=\sup _{x \in \mathbb{R}^{3}}|x|^{2} V(x)+\int \frac{\mathrm{d} x}{|x|} V(x)
$$

to be sufficiently small. In the present paper, we remove this condition and consider arbitrary repulsive potentials $V \geq 0$, with the fast decay property $V(x) \leq C\langle x\rangle^{-\sigma}$ for some $\sigma>5$ (here $\left.\langle x\rangle=\left(1+x^{2}\right)^{1 / 2}\right)$.

The removal of the smallness condition requires completely new ideas. The main challenge in the derivation of the Gross-Pitaevskii equation is to identify and resolve the short scale correlation structure in the $N$-body wave function. In [12] we achieved this by locally factoring out the solution of the zero energy scattering equation (1.5). This approach, however, is not sufficiently precise for a large interaction potential. In the present paper we propose an intrinsic characterization of the correlation structure in terms of the two-particle scattering wave operator. More precisely, we prove that the action of the wave operator in the relative coordinate $x_{i}-x_{j}$ regularizes $\psi_{N, t}$ in this variable. This regularity is essential to control the convergence of the many-body interaction to the local on-site nonlinearity in the limiting equation (1.8). An a priori estimate leading to this regularity will be obtained from the conservation of the second moment of the energy, i.e. from $\left\langle\psi_{N, t}, H_{N}^{2} \psi_{N, t}\right\rangle=\left\langle\psi_{N}, H_{N}^{2} \psi_{N}\right\rangle$. This a priori bound, however, only controls a specific combination of two derivatives, $\nabla_{x_{i}} \cdot \nabla_{x_{j}}$, acting on the regularized wave function. We thus need to establish a new Poincaré-type inequality involving only this combination of derivatives. In the next section we discuss the main ideas of our new approach.

\section{Resolution of the CORRELATION STRUCtURE FOR LARGE POTENTIAL}

As in [12, the general strategy of our proof is based on the study of solutions of the BBGKY hierarchy of equations for the marginal densities $\gamma_{N, t}^{(k)}$ associated with the solution of the $N$-particle Schrödinger equation (1.3):

$$
\begin{aligned}
i \partial_{t} \gamma_{N, t}^{(k)}= & \sum_{j=1}^{k}\left[-\Delta_{j}, \gamma_{N, t}^{(k)}\right]+\sum_{i<j}^{k}\left[V_{N}\left(x_{i}-x_{j}\right), \gamma_{N, t}^{(k)}\right] \\
& +(N-k) \sum_{j=1}^{k} \operatorname{Tr}_{k+1}\left[V_{N}\left(x_{j}-x_{k+1}\right), \gamma_{N, t}^{(k+1)}\right]
\end{aligned}
$$

for $k=1, \ldots, N$. Here $\operatorname{Tr}_{k+1}$ denotes the partial trace over the $(k+1)$-th variable, and we use the convention that $\gamma_{N, t}^{(k)}=0$ for $k=N+1$. The main observation is the fact that limit points $\left\{\gamma_{\infty, t}^{(k)}\right\}_{k \geq 1}$ of the families $\left\{\gamma_{N, t}^{(k)}\right\}_{k=1}^{N}$ (with respect to an appropriate topology) are a solution of the infinite hierarchy of equations

$$
i \partial_{t} \gamma_{\infty, t}^{(k)}=\sum_{j=1}^{k}\left[-\Delta_{j}, \gamma_{\infty, t}^{(k)}\right]+8 \pi a_{0} \sum_{j=1}^{k} \operatorname{Tr}_{k+1}\left[\delta\left(x_{j}-x_{k+1}\right), \gamma_{\infty, t}^{(k+1)}\right]
$$


It is easy to check that the product ansatz $\gamma_{\infty, t}^{(k)}=\left|\varphi_{t}\right\rangle\left\langle\left.\varphi_{t}\right|^{\otimes k}\right.$ satisfies (2.2) if and only if $\varphi_{t}$ solves the Gross-Pitaevskii equation (1.8). Therefore, to conclude the proof of (1.7), it suffices to show that: 1) every limit point of the family $\left\{\gamma_{N, t}^{(k)}\right\}_{k=1}^{N}$ is a solution of the infinite hierarchy (2.2), and 2) the solution to (2.2) is unique. This strategy has already been used to derive the nonlinear Hartree equations for the effective dynamics of so-called mean-field systems (see [27, 13, 4, 9]) to derive the cubic nonlinear Schrödinger equation with different (and simpler) scalings of the interaction potential (see [8, 11]) and to derive the nonlinear Schrödinger equation in a one-dimensional setting (see [1, 2]). We remark that the first derivation of the Hartree equation was obtained using a different method in [17, 14]. With this method the speed of convergence was recently estimated in [25].

In all works based on the BBGKY hierarchy, the key step consists of finding an appropriate norm and space of density matrices with which to work. On the one hand, the topology has to be sufficiently strong to guarantee the convergence of (2.1) to (2.2) and the space has to be sufficiently small to guarantee the uniqueness of the solution to (2.2). On the other hand, the norm defining this space cannot be too strong since we have to prove, via an a priori bound, that limit points of the sequence $\gamma_{N, t}^{(k)}$ belong to this space.

In [12, we use an appropriate Poincaré-type inequality to prove the convergence of (2.1) to (2.2). To do that, we need a control on a mixed Sobolev norm on $\gamma_{N, t}^{(k+1)}$ and $\gamma_{\infty, t}^{(k+1)}$ with at least two derivatives (note that the commutator with the delta function in (2.2) is even ill-defined unless some regularity is known on $\left.\gamma_{\infty, t}^{(k+1)}\right)$. However, due to the singularity of the interaction potential, it turns out that the solution of the Schrödinger equation $\psi_{N, t}$ develops a short-scale correlation structure, living on a length-scale $O(1 / N)$, which causes the Sobolev norms with two or more derivatives to blow up as $N \rightarrow \infty$.

Instead of considering derivatives of $\psi_{N, t}$, we therefore prove an a priori bound of the form $\int\left|\nabla_{1} \nabla_{2} \phi_{12, N}(t)\right|^{2} \leq C$ on the $N$-body function $\phi_{12, N}(t)=\psi_{N, t} /\left(1-w_{12}\right)$ obtained from the original wave function after factoring out the singular short-scale structure. Here $1-w_{12}=1-w_{N}\left(x_{1}-x_{2}\right)$, where $f_{N}=1-w_{N}$ is the zeroenergy scattering solution to $\left(-\Delta+(1 / 2) V_{N}\right) f_{N}=0$. Note that, by simple scaling, $w_{N}(x)=w(N x)$, where $f(x)=1-w(x)$ is the zero-energy scattering solution to the unscaled equation $(-\Delta+(1 / 2) V) f=0$. It turns out that $\int\left|\nabla_{1} \nabla_{2} \psi_{N, t}\right|^{2}$ grows with $N$, but $\int\left|\nabla_{1} \nabla_{2} \phi_{12, N}(t)\right|^{2}$ remains bounded. Although $\psi_{N, t}$ and $\phi_{12, N}(t)$ behave very differently in the mixed Sobolev norm, their difference in the $L^{2}$-norm vanishes in the $N \rightarrow \infty$ limit due to the scaling $w_{N}(x)=w(N x)$. This allows us to obtain control on the mixed Sobolev norm of $\gamma_{\infty, t}$, despite the fact that it is defined as a limit of $\gamma_{N, t}$ only in the weak topology of trace class operators. Moreover, the boundedness of $\int\left|\nabla_{1} \nabla_{2} \phi_{12, N}(t)\right|^{2}$ explains the emergence of the scattering length in (2.2).

The proof of $\int\left|\nabla_{1} \nabla_{2} \phi_{12, N}(t)\right|^{2} \leq C$ relies on the conservation of $H_{N}^{2}$ along the time evolution and on the key inequality

$$
\left\langle\psi_{N},\left(H_{N}+N\right)^{2} \psi_{N}\right\rangle \geq C N^{2} \int\left|\nabla_{1} \nabla_{2} \frac{\psi_{N}}{1-w_{12}}\right|^{2},
$$

valid for all $\psi_{N} \in L_{s}^{2}\left(\mathbb{R}^{3 N}\right)$. 
To show the uniqueness of the solution of the infinite hierarchy (2.2), on the other hand, more information on the limiting densities $\gamma_{\infty, t}^{(k)}$ is needed; more precisely, uniqueness was proven in [11] under the assumption that

$$
\operatorname{Tr}\left(1-\Delta_{1}\right) \ldots\left(1-\Delta_{k}\right) \gamma_{\infty, t}^{(k)} \leq C^{k}
$$

for all $k \geq 1$. Because of the singular short-scale structure characterizing the solution of the Schrödinger equation for finite $N$, the densities $\gamma_{N, t}^{(k)}$ do not satisfy (2.4). To circumvent this problem, we derived in [12] a higher order energy estimate of the form

$$
\left\langle\psi_{N},\left(H_{N}+N\right)^{k} \psi_{N}\right\rangle \geq C^{k} N^{k} \int \mathrm{d} \mathbf{x} \Theta_{k-1}(\mathbf{x})\left|\nabla_{1} \ldots \nabla_{k} \psi_{N}(\mathbf{x})\right|^{2}
$$

for all $k \geq 1$, where $\Theta_{k-1}(\mathbf{x})$ is a cutoff function vanishing (up to exponentially small contributions) in regions where a second particle comes close to $x_{j}$, for some $j \leq k-1$ (see Section 7 for the precise definition of the cutoff). This higher order energy estimate provides a control on the $L^{2}$ norm of the mixed derivatives $\nabla_{1} \ldots \nabla_{k} \psi_{N, t}$ restricted on regions with no other particle close to $x_{1}, \ldots, x_{k-1}$. Choosing $\Theta_{k-1}$ to vanish in a sufficiently small volume, it was possible to remove the cutoffs in the weak limit $N \rightarrow \infty$ and to obtain the a priori bounds (2.4) on the limit points $\left\{\gamma_{\infty, t}^{(k)}\right\}_{k \geq 1}$.

The estimates (2.3) and (2.5) were therefore the two main ingredients used in 12 to control the singularity of the interaction potential $V_{N}$ and the singular short scale structure of $\psi_{N, t}$. In [12, both of these estimates heavily relied on the smallness of the parameter $\rho$ introduced in (1.10). Therefore, although the general strategy of the current paper is similar to the one used in [12, the removal of the smallness condition on $\rho$ requires completely new ideas, which we now explain.

In [12, the energy estimate (2.3) was obtained from an identity of the form

$$
\left\langle\psi_{N}, H_{N}^{2} \psi_{N}\right\rangle=N(N-1) \int\left|\nabla_{1} \nabla_{2} \phi_{12}\right|^{2}\left(1-w_{12}\right)^{2}+N(N-1) \int \nabla_{1} \bar{\phi}_{12} \cdot g_{12} \nabla_{2} \phi_{12}+\Omega,
$$

where $\phi_{12}=\psi_{N} /\left(1-w_{12}\right)$. Here $g_{12}$ is an explicit matrix involving the Hessian of $w_{12}$ and squares of its first derivatives, and $\Omega$ contains irrelevant terms. The following bound is essentially optimal for the size of $g_{12}$ :

$$
\left|g_{12}\right| \leq \frac{C \rho}{\left|x_{1}-x_{2}\right|^{2}}
$$

The best strategy is then to estimate

$$
\begin{aligned}
\int \nabla_{1} \bar{\phi}_{12} \cdot g_{12} \nabla_{2} \phi_{12} & \geq-C \rho \int \frac{1}{\left|x_{1}-x_{2}\right|^{2}}\left(\left|\nabla_{1} \phi_{12}\right|^{2}+\left|\nabla_{2} \phi_{12}\right|^{2}\right) \\
& \geq-C \rho \int\left|\nabla_{1} \nabla_{2} \phi_{12}\right|^{2},
\end{aligned}
$$

where we used Hardy's inequality in the last step. This term can be absorbed in the first (positive) term in (2.6) only if $\rho$ is sufficiently small. A closer inspection 
of the structure of the terms in $\Omega$ reveals that, although some of them are positive, they cannot compensate for the negative term (2.7). After estimating them from below at the expense of adding lower order terms to $H_{N}^{2}$, we get the desired bound

$$
\int\left|\nabla_{1} \nabla_{2} \phi_{12}\right|^{2} \leq C N^{-2}\left\langle\psi_{N},\left(H_{N}+N\right)^{2} \psi_{N}\right\rangle .
$$

The first main idea of the current paper is to prove the following replacement for (2.8) (see Proposition 5.2):

$$
\int\left|\nabla_{1} \cdot \nabla_{2} W_{12}^{*} \psi_{N}\right|^{2} \leq C N^{-2}\left\langle\psi_{N},\left(H_{N}+N\right)^{2} \psi_{N}\right\rangle
$$

where $W_{12}$ denotes the wave operator of the one particle Hamiltonian $-\Delta+\frac{1}{2} V_{N}$ acting on the difference variable $x_{1}-x_{2}$. Note two main differences between (2.8) and (2.9). First, $W_{12}^{*} \psi_{N}$ replaces the function $\phi_{12, N}=\psi_{N} /\left(1-w_{12}\right)$ that can be considered as a first order approximation to $W_{12}^{*} \psi_{N}$. Second, instead of controlling the full mixed second derivative, $\nabla_{1} \nabla_{2}$, as in (2.8), the new bound controls only $\nabla_{1} \cdot \nabla_{2}$. Although the control on $\nabla_{1} \cdot \nabla_{2}$ is in general much weaker than the control on $\nabla_{1} \nabla_{2}$, in the radial direction of the relative coordinate $x_{1}-x_{2}$ the new bound is as strong as the former one.

Both differences cause substantial difficulties in proving that 2.1 indeed converges to (2.2). First, instead of working with a relatively explicit function $w_{12}$ and using its fairly straighforward properties summarized in Lemma 5.1 of 12, now analogous properties have to be established for the wave operator. Second, the lack of the control on the full mixed second derivatives impedes using the Sobolev-type operator inequality

$$
V\left(x_{1}-x_{2}\right) \leq C\|V\|_{1}\left(1-\Delta_{1}\right)\left(1-\Delta_{2}\right)
$$

that was crucial in controlling many error terms. We have found a replacement for this inequality (Lemma 10.1):

$$
V\left(x_{1}-x_{2}\right) \leq C\|V\|_{1}\left(\left(\nabla_{1} \cdot \nabla_{2}\right)^{2}-\Delta_{1}-\Delta_{2}+1\right)
$$

that uses only the $\nabla_{1} \cdot \nabla_{2}$ combination in the highest order term. Similarly to Lemma 7.2 of 12 , we are also able to improve (2.10) to a Poincaré-type inequality $\left\langle\varphi,\left(h_{\alpha}\left(x_{1}-x_{2}\right)-\delta\left(x_{1}-x_{2}\right)\right) \psi\right\rangle \rightarrow 0$ as $\alpha \rightarrow 0$, where $h_{\alpha}(x)=\alpha^{-3} h(x / \alpha)$ (with $\int h(x) \mathrm{d} x=1$ ) is an approximate Dirac delta function on the scale $\alpha$ (see Lemma 10.2). This is necessary to control the convergence of (2.1) to (2.2).

The second main novelty of this paper is a proof for the higher order derivative estimates (2.5) in the large potential regime (Proposition 17.1). Although the main conclusion is the same as Proposition 5.3 in [12, the proof does not require the smallness of $\rho$. The proof in 12 started from the trivial energy estimate $\sum_{j=1}^{N}-\Delta_{j} \leq H_{N}$ and the estimate (2.8) on $H_{N}^{2}$, and it used an induction on the exponent to pass from $\left(H_{N}+N\right)^{k}$ to $\left(H_{N}+N\right)^{k+2}$. The step-two induction allowed us to start the induction estimate with

$$
\left(H_{N}+N\right)^{k+2} \geq\left(H_{N}+N\right) \nabla_{1}^{*} \ldots \nabla_{k}^{*} \Theta \nabla_{k} \ldots \nabla_{1}\left(H_{N}+N\right)
$$

and then we commuted the two $H_{N}+N$ factors through to the middle and used 
the $\left(H_{N}+N\right)^{2}$ estimate (2.8). The weaker $H_{N}^{2}$ estimate (2.9) however does not allow us to regain control on full derivatives, so pursuing this path would, at best, yield control on some complicated combinations of partial derivatives.

In this paper we establish higher order derivative estimates by a step-one induction, i.e. passing from $\left(H_{N}+N\right)^{k}$ to $\left(H_{N}+N\right)^{k+1}$. This eliminates using the $H_{N}^{2}$ estimate with incomplete derivatives, but the price is that instead of (2.11) we have to work with

$$
\left(H_{N}+N\right)^{k+1} \geq\left(H_{N}+N\right)^{1 / 2} \nabla_{1}^{*} \ldots \nabla_{k}^{*} \Theta \nabla_{k} \ldots \nabla_{1}\left(H_{N}+N\right)^{1 / 2}
$$

in the induction step, i.e. we have to commute the square root of the Hamiltonian through the derivatives. The technical complications involved with the square root turn out to be reasonably easily managable, so that this new method actually provides a simpler proof than in [12] for the higher energy estimates even when $\rho$ is small.

Notation. Throughout the paper we will use the notation $\mathbf{x}=\left(x_{1}, \ldots, x_{N}\right) \in \mathbb{R}^{3 N}$, and, for $k=1, \ldots, N, \mathbf{x}_{k}=\left(x_{1}, \ldots, x_{k}\right), \mathbf{x}_{k}^{\prime}=\left(x_{1}^{\prime}, \ldots, x_{k}^{\prime}\right) \in \mathbb{R}^{3 k}$, and $\mathbf{x}_{N-k}=$ $\left(x_{k+1}, \ldots, x_{N}\right) \in \mathbb{R}^{3(N-k)}$. The notation $\|\cdot\|$ indicates the $L^{2}$-norm if the argument is a function, and it denotes the operator norm (from $L^{2}$ to $L^{2}$ ) if the argument is an operator. For $1 \leq p \leq \infty,\|f\|_{p}$ indicates the $L^{p}$-norm of $f$. Moreover, we will use $\nabla_{j}$ and $\Delta_{j}$ as shorthand notation for $\nabla_{x_{j}}$ and, respectively, $\Delta_{x_{j}}$. If $A$ is an operator acting on $L^{2}\left(\mathbb{R}^{3 k}\right)$, we will denote its kernel by $A\left(\mathbf{x}_{k} ; \mathbf{x}_{k}^{\prime}\right)$. The letter $C$ denotes universal constants that may depend on $V$ and on the $H^{1}$-norm of the initial one particle wave function $\varphi$, but is independent of $N$.

\section{MAIN THEOREM}

The following theorem is the main result of this paper.

Theorem 3.1. Suppose that $V \geq 0$, with $V(-x)=V(x)$ and $V(x) \leq C\langle x\rangle^{-\sigma}$, for some $\sigma>5$ and for all $x \in \mathbb{R}^{3}$. Assume that the family $\psi_{N} \in L_{s}^{2}\left(\mathbb{R}^{3 N}\right)$, with $\left\|\psi_{N}\right\|=1$ for all $N$, has finite energy per particle, in the sense that

$$
\left\langle\psi_{N}, H_{N} \psi_{N}\right\rangle \leq C N
$$

and that it exhibits complete Bose-Einstein condensation in the sense that the oneparticle marginal $\gamma_{N}^{(1)}$ associated with $\psi_{N}$ satisfies

$$
\gamma_{N}^{(1)} \rightarrow|\varphi\rangle\langle\varphi| \quad \text { in the trace norm topology as } N \rightarrow \infty
$$

for some $\varphi \in H^{1}\left(\mathbb{R}^{3}\right)$. Then, for every $k \geq 1$ and $t \in \mathbb{R}$, we have

$$
\gamma_{N, t}^{(k)} \rightarrow\left|\varphi_{t}\right\rangle\left\langle\left.\varphi_{t}\right|^{\otimes k}\right.
$$

as $N \rightarrow \infty$, in the trace norm. Here $\varphi_{t}$ is the solution of the nonlinear GrossPitaevskii equation

$$
i \partial_{t} \varphi_{t}=-\Delta \varphi_{t}+8 \pi a_{0}\left|\varphi_{t}\right|^{2} \varphi_{t}
$$

with initial data $\varphi_{t=0}=\varphi$. 
Remark. Note that the condition $V(x) \leq C\langle x\rangle^{-\sigma}$ for some $\sigma>5$ and for all $x \in \mathbb{R}^{3}$ is only required to apply the result of Yajima in [29], which guarantees that the wave operator $W$ associated with the Hamiltonian $\mathfrak{h}=-\Delta+(1 / 2) V$ maps $L^{p}\left(\mathbb{R}^{3}\right)$ into itself, for all $1 \leq p \leq \infty$ (see Proposition 5.1). If one knows, by different means, that $\|W\|_{L^{p} \rightarrow L^{p}}<\infty$ (it suffices to know it for $p=1$ and $p=\infty$ ), then it would be enough to assume that $V \in L^{1}\left(\mathbb{R}^{3},\left(1+|x|^{2}\right) \mathrm{d} x\right) \cap L^{2}\left(\mathbb{R}^{3}, \mathrm{~d} x\right)$.

Remark. Compared with our previous result in [12, here we do not require the potential $V$ to be spherical symmetric (we only need that $V(-x)=V(x)$ ).

Remark. The fact that $\varphi \in H^{1}\left(\mathbb{R}^{3}\right)$ does not need to be assumed separately, since it already follows from the assumption (3.1).

To prove this theorem we will use an approximation argument and the following theorem, which proves Theorem 3.1 for a smaller class of initial $N$-particle wave functions.

Theorem 3.2. Assume the same conditions on the potential $V$ as in Theorem 3.1. Suppose moreover that $\left|\nabla^{\alpha} V(x)\right| \leq C$ for all multi-indices $\alpha$ with $|\alpha| \leq 2$. Assume that the family $\psi_{N} \in L^{2}\left(\mathbb{R}^{3 N}\right)$, with $\left\|\psi_{N}\right\|=1$, is such that

$$
\left\langle\psi_{N}, H_{N}^{k} \psi_{N}\right\rangle \leq C^{k} N^{k}
$$

for all $k \in \mathbb{N}$ and that

$$
\gamma_{N}^{(1)} \rightarrow|\varphi\rangle\langle\varphi| \quad \text { in the trace norm topology as } N \rightarrow \infty
$$

for some $\varphi \in H^{1}\left(\mathbb{R}^{3}\right)$. Then, for every $k \geq 1$ and $t \in \mathbb{R}$,

$$
\gamma_{N, t}^{(k)} \rightarrow\left|\varphi_{t}\right\rangle\left\langle\left.\varphi_{t}\right|^{\otimes k}\right.
$$

as $N \rightarrow \infty$, in the trace norm. Here $\varphi_{t}$ is the solution of the nonlinear GrossPitaevskii equation (3.3) with initial data $\varphi_{t=0}=\varphi$.

\section{Proof of the MAIN THEOREM}

In this section we present the proof of Theorem 3.2 and we show how it implies Theorem 3.1 making use of several key propositions, whose proofs are deferred to subsequent sections.

We start by defining an appropriate space of time-dependent density matrices. To use the Arzela-Ascoli compactness argument, we will need to establish the concept of uniform continuity in this space, thus we have to metrize the weak* topology.

Let $\mathcal{K}_{k} \equiv \mathcal{K}\left(L^{2}\left(\mathbb{R}^{3 k}\right)\right)$ denote the space of compact operators on $L^{2}\left(\mathbb{R}^{3 k}\right)$ equipped with the operator norm topology and let $\mathcal{L}_{k}^{1} \equiv \mathcal{L}^{1}\left(L^{2}\left(\mathbb{R}^{3 k}\right)\right)$ denote the space of trace class operators on $L^{2}\left(\mathbb{R}^{3 k}\right)$ equipped with the trace norm. It is well known that $\mathcal{L}_{k}^{1}$ is the dual of $\mathcal{K}_{k}$. Since $\mathcal{K}_{k}$ is separable, we can fix a dense countable subset of the unit ball of $\mathcal{K}_{k}$; we denote it by $\left\{J_{i}^{(k)}\right\}_{i \geq 1} \in \mathcal{K}_{k}$, with $\left\|J_{i}^{(k)}\right\| \leq 1$ for all $i \geq 1$. Using the operators $J_{i}^{(k)}$ we define the following metric on the space $\mathcal{L}_{k}^{1} \equiv \mathcal{L}^{1}\left(L^{2}\left(\mathbb{R}^{3 k}\right)\right)$ : for $\gamma^{(k)}, \bar{\gamma}^{(k)} \in \mathcal{L}_{k}^{1}$ we set

$$
\eta_{k}\left(\gamma^{(k)}, \bar{\gamma}^{(k)}\right):=\sum_{i=1}^{\infty} 2^{-i}\left|\operatorname{Tr} J_{i}^{(k)}\left(\gamma^{(k)}-\bar{\gamma}^{(k)}\right)\right| .
$$


Then the topology induced by the metric $\eta_{k}$ and the weak* topology are equivalent on the unit ball of $\mathcal{L}_{k}^{1}$ (see [26], Theorem 3.16) and hence on any ball of finite radius as well. In other words, a uniformly bounded sequence $\gamma_{N}^{(k)} \in \mathcal{L}_{k}^{1}$ converges to $\gamma^{(k)} \in \mathcal{L}_{k}^{1}$ with respect to the weak* topology, if and only if $\eta_{k}\left(\gamma_{N}^{(k)}, \gamma^{(k)}\right) \rightarrow 0$ as $N \rightarrow \infty$.

For a fixed $T>0$, let $C\left([0, T], \mathcal{L}_{k}^{1}\right)$ be the space of functions of $t \in[0, T]$ with values in $\mathcal{L}_{k}^{1}$ which are continuous with respect to the metric $\eta_{k}$. On $C\left([0, T], \mathcal{L}_{k}^{1}\right)$ we define the metric

$$
\widehat{\eta}_{k}\left(\gamma^{(k)}(\cdot), \bar{\gamma}^{(k)}(\cdot)\right):=\sup _{t \in[0, T]} \eta_{k}\left(\gamma^{(k)}(t), \bar{\gamma}^{(k)}(t)\right) .
$$

Finally, we denote by $\tau_{\text {prod }}$ the topology on the space $\bigoplus_{k \geq 1} C\left([0, T], \mathcal{L}_{k}^{1}\right)$ given by the product of the topologies generated by the metrics $\widehat{\eta}_{k}$ on $C\left([0, T], \mathcal{L}_{k}^{1}\right)$.

Proof of Theorem 3.2. The proof is divided in four steps.

Step 1. Compactness of $\Gamma_{N, t}=\left\{\gamma_{N, t}^{(k)}\right\}_{k \geq 1}$. We fix $T>0$ and work on the interval $t \in[0, T]$. Negative times can be handled analogously.

In Theorem 6.1 we show that the sequence $\Gamma_{N, t}^{(k)}=\left\{\gamma_{N, t}^{(k)}\right\}_{k \geq 1} \in \bigoplus_{k \geq 1} C\left([0, T], \mathcal{L}_{k}^{1}\right)$ is compact with respect to the product topology $\tau_{\text {prod }}$ defined above (we use the convention that $\gamma_{N, t}^{(k)}=0$ if $\left.k>N\right)$. It also follows from Theorem 6.1 that any limit point $\Gamma_{\infty, t}=\left\{\gamma_{\infty, t}^{(k)}\right\}_{k \geq 1} \in \bigoplus_{k \geq 1} C\left([0, T], \mathcal{L}_{k}^{1}\right)$ is such that, for every $k \geq 1$, $\gamma_{\infty, t}^{(k)} \geq 0$, and $\gamma_{\infty, t}^{(k)}$ is symmetric w.r.t. permutations.

Using higher order energy estimates from Proposition 7.1 we show in Theorem 7.3 that an arbitrary limit point $\Gamma_{\infty, t}=\left\{\gamma_{\infty, t}^{(k)}\right\}_{k \geq 1}$ of the sequence $\Gamma_{N, t}^{(k)}$ (with respect to the topology $\tau_{\text {prod }}$ ) is such that

$$
\operatorname{Tr}\left(1-\Delta_{1}\right) \ldots\left(1-\Delta_{k}\right) \gamma_{\infty, t}^{(k)} \leq C^{k}
$$

for every $t \in[0, T]$ and every $k \geq 1$.

Step 2. Convergence to the infinite hierarchy. In Theorem 8.1 we prove that any limit point $\Gamma_{\infty, t}=\left\{\gamma_{\infty, t}^{(k)}\right\}_{k \geq 1} \in \bigoplus_{k \geq 1} C\left([0, T], \mathcal{L}_{k}^{1}\right)$ of $\Gamma_{N, t}=\left\{\gamma_{N, t}^{(k)}\right\}_{k \geq 1}$ with respect to the product topology $\tau_{\text {prod }}$ is a solution of the infinite hierarchy of integral equations $(k=1,2, \ldots)$

$$
\gamma_{\infty, t}^{(k)}=\mathcal{U}^{(k)}(t) \gamma_{\infty, 0}^{(k)}-8 \pi i a_{0} \sum_{j=1}^{k} \int_{0}^{t} \mathrm{~d} s \mathcal{U}^{(k)}(t-s) \operatorname{Tr}_{k+1}\left[\delta\left(x_{j}-x_{k+1}\right), \gamma_{\infty, s}^{(k+1)}\right]
$$

with initial data $\gamma_{\infty, 0}^{(k)}=|\varphi\rangle\left\langle\left.\varphi\right|^{\otimes k}\right.$ (where $\varphi \in H^{1}\left(\mathbb{R}^{3}\right)$ has been introduced in (3.5)). Here $\operatorname{Tr}_{k+1}$ denotes the partial trace over the $(k+1)$-th particle, and $\mathcal{U}^{(k)}(t)$ is the free evolution whose action on $k$-particle density matrices is given by

$$
\mathcal{U}^{(k)}(t) \gamma^{(k)}:=e^{i t \sum_{j=1}^{k} \Delta_{j}} \gamma^{(k)} e^{-i t \sum_{j=1}^{k} \Delta_{j}} .
$$

We remark next that the family of factorized densities,

$$
\gamma_{t}^{(k)}=\left|\varphi_{t}\right\rangle\left\langle\left.\varphi_{t}\right|^{\otimes k},\right.
$$

is a solution of the infinite hierarchy (4.4) if $\varphi_{t}$ is the solution of the nonlinear Gross-Pitaevskii equation (3.3) with initial data $\varphi_{t=0}=\varphi$. The nonlinear Schrödinger equation (3.3) is well-posed in $H^{1}\left(\mathbb{R}^{3}\right)$, and it conserves the energy, 
$\mathcal{E}(\varphi):=\frac{1}{2} \int|\nabla \varphi|^{2}+4 \pi a_{0} \int|\varphi|^{4}$. From $\varphi \in H^{1}\left(\mathbb{R}^{3}\right)$, we thus obtain that $\varphi_{t} \in$ $H^{1}\left(\mathbb{R}^{3}\right)$ for every $t \in \mathbb{R}$, with a uniformly bounded $H^{1}$-norm. Therefore

$$
\operatorname{Tr}\left(1-\Delta_{1}\right) \ldots\left(1-\Delta_{k}\right)\left|\varphi_{t}\right\rangle\left\langle\left.\varphi_{t}\right|^{\otimes k} \leq\left\|\varphi_{t}\right\|_{H^{1}}^{k} \leq C^{k}\right.
$$

for all $t \in \mathbb{R}$ and a constant $C$ only depending on the $H^{1}$-norm of $\varphi$. For the wellposedness of the subcritical nonlinear Schrödinger equation (3.3) in $H^{1}$, see, e.g., [21. We remark that the well-posedness has been established even for the critical (quintic) nonlinear Schrödinger equation in [15, 16, 28, for small data and in [5, 6] for large data.

Step 3. Uniqueness of the solution to the infinite hierarchy. In Section 9 of [1] we proved the following theorem, which states the uniqueness of solution to the infinite hierarchy (4.4) in the space of densities satisfying the a priori bound (4.3). The proof of this theorem is based on a diagrammatic expansion of the solution of (4.4). Remark that the uniqueness of the infinite hierarchy in a different space of densities was proven in 22.

Theorem 4.1 (Theorem 9.1 of [1]). Suppose $\Gamma=\left\{\gamma^{(k)}\right\}_{k \geq 1} \in \bigoplus_{k \geq 1} \mathcal{L}_{k}^{1}$ is such that

$$
\operatorname{Tr}\left(1-\Delta_{1}\right) \ldots\left(1-\Delta_{k}\right) \gamma^{(k)} \leq C^{k} .
$$

Then, for any fixed $T>0$, there exists at most one solution $\Gamma_{t}=\left\{\gamma_{t}^{(k)}\right\}_{k \geq 1} \in$ $\bigoplus_{k \geq 1} C\left([0, T], \mathcal{L}_{k}^{1}\right)$ of (4.4) such that

$$
\operatorname{Tr}\left(1-\Delta_{1}\right) \ldots\left(1-\Delta_{k}\right) \gamma_{t}^{(k)} \leq C^{k}
$$

for all $t \in[0, T]$ and for all $k \geq 1$.

Step 4. Conclusion of the proof. From Step 2 and Step 3 it follows that the sequence $\Gamma_{N, t}=\left\{\gamma_{N, t}^{(k)}\right\}_{k \geq 1} \in \bigoplus_{k \geq 1} C\left([0, T], \mathcal{L}_{k}^{1}\right)$ is convergent with respect to the product topology $\tau_{\text {prod }}$; in fact a compact sequence with only one limit point is always convergent. Since the family of densities $\Gamma_{t}=\left\{\gamma_{t}^{(k)}\right\}_{k \geq 1}$ defined in (4.6) satisfies (4.7) and is a solution of (4.4), it follows that $\Gamma_{N, t} \rightarrow \Gamma_{t}$ w.r.t. the topology $\tau_{\text {prod. }}$. In particular this implies that, for every fixed $k \geq 1$ and $t \in[0, T], \gamma_{N, t}^{(k)} \rightarrow$ $\left|\varphi_{t}\right\rangle\left\langle\left.\varphi_{t}\right|^{\otimes k}\right.$ with respect to the weak* topology of $\mathcal{L}_{k}^{1}$, and thus, by a standard argument, also in the trace-norm topology. This completes the proof of Theorem 3.2 .

Next we prove Theorem 3.1. To this end we have to combine Theorem 3.2 with an approximation argument for the initial $N$-particle wave function, which is needed to make sure that the energy condition (3.4) is satisfied. This argument was already used in [12]; we present it here for completeness.

Proof of Theorem 3.1. We assume here that, as in Theorem 3.2, the interaction potential $V$ is such that $\left|\nabla^{\alpha} V(x)\right| \leq C$ for all multi-indices $\alpha$ with $|\alpha| \leq 2$. We show how to remove this condition in Appendix B.

Fix $\kappa>0$ and $\chi \in C_{0}^{\infty}(\mathbb{R})$, with $0 \leq \chi \leq 1, \chi(s)=1$, for $0 \leq s \leq 1$, and $\chi(s)=0$ if $s \geq 2$. We define the regularized initial wave function

$$
\widetilde{\psi}_{N}:=\frac{\chi\left(\kappa H_{N} / N\right) \psi_{N}}{\left\|\chi\left(\kappa H_{N} / N\right) \psi_{N}\right\|}
$$


and we denote by $\widetilde{\psi}_{N, t}$ the solution of the Schrödinger equation (1.3) with initial data $\widetilde{\psi}_{N}$. Denote by $\widetilde{\Gamma}_{N, t}=\left\{\widetilde{\gamma}_{N, t}^{(k)}\right\}_{k=1}^{\infty}$ the family of marginal densities associated

with $\widetilde{\psi}_{N, t}$. By convention, we set $\widetilde{\gamma}_{N, t}^{(k)}:=0$ if $k>N$. The tilde in the notation indicates the dependence on the cutoff parameter $\kappa$. In Proposition 9.1, part i), we prove that

$$
\left\langle\widetilde{\psi}_{N, t}, H_{N}^{k} \widetilde{\psi}_{N, t}\right\rangle \leq \widetilde{C}^{k} N^{k}
$$

if $\kappa>0$ is sufficiently small (the constant $\widetilde{C}$ depends on $\kappa$ ). Moreover, in part iii) of Proposition 9.1, we show that, for every $J^{(k)} \in \mathcal{K}_{k}$,

$$
\operatorname{Tr} J^{(k)}\left(\widetilde{\gamma}_{N}^{(k)}-|\varphi\rangle\left\langle\left.\varphi\right|^{\otimes k}\right) \rightarrow 0\right.
$$

as $N \rightarrow \infty$. From (4.10) and (4.11), the assumptions (3.4) and (3.5) of Theorem 3.2 are satisfied by the regularized wave function $\widetilde{\psi}_{N}$ and by the regularized marginal densities $\widetilde{\gamma}_{N, t}^{(k)}$. Applying Theorem 3.2, we obtain that, for every $t \in \mathbb{R}$ and $k \geq 1$,

$$
\widetilde{\gamma}_{N, t}^{(k)} \rightarrow\left|\varphi_{t}\right\rangle\left\langle\left.\varphi_{t}\right|^{\otimes k}\right.
$$

where $\varphi_{t}$ is the solution of (3.3) with initial data $\varphi_{t=0}=\varphi$.

It remains to prove that the densities $\gamma_{N, t}^{(k)}$ associated with the original wave function $\psi_{N, t}$ (without cutoff $\kappa$ ) converge and have the same limit as the regularized densities $\widetilde{\gamma}_{N, t}^{(k)}$. This follows from Proposition 9.1, part ii), where we prove that

$$
\left\|\psi_{N, t}-\widetilde{\psi}_{N, t}\right\|=\left\|\psi_{N}-\widetilde{\psi}_{N}\right\| \leq C \kappa^{1 / 2}
$$

for a constant $C$ independent of $N$ and $\kappa$. This implies that, for every $J^{(k)} \in \mathcal{K}_{k}$, we have

$$
\left|\operatorname{Tr} J^{(k)}\left(\gamma_{N, t}^{(k)}-\widetilde{\gamma}_{N, t}^{(k)}\right)\right| \leq C\left\|J^{(k)}\right\| \kappa^{1 / 2}
$$

Therefore, for every fixed $k \geq 1, t \in \mathbb{R}, J^{(k)} \in \mathcal{K}_{k}$, we have

$$
\begin{aligned}
& \left|\operatorname { T r } J ^ { ( k ) } \left(\gamma_{N, t}^{(k)}-\left|\varphi_{t}\right\rangle\left\langle\left.\varphi_{t}\right|^{\otimes k}\right) \mid\right.\right. \\
& \quad \leq\left|\operatorname{Tr} J^{(k)}\left(\gamma_{N, t}^{(k)}-\widetilde{\gamma}_{N, t}^{(k)}\right)\right|+\left|\operatorname { T r } J ^ { ( k ) } \left(\widetilde{\gamma}_{N, t}^{(k)}-\left|\varphi_{t}\right\rangle\left\langle\left.\varphi_{t}\right|^{\otimes k}\right) \mid\right.\right. \\
& \quad \leq C\left\|J^{(k)}\right\| \kappa^{1 / 2}+\left|\operatorname { T r } J ^ { ( k ) } \left(\widetilde{\gamma}_{N, t}^{(k)}-\left|\varphi_{t}\right\rangle\left\langle\left.\varphi_{t}\right|^{\otimes k}\right) \mid .\right.\right.
\end{aligned}
$$

Since $\kappa>0$ was arbitrary, it follows from (4.12) that the l.h.s. of (4.14) converges to zero as $N \rightarrow \infty$. This implies that, for arbitrary $k \geq 1$ and $t \in \mathbb{R}, \gamma_{N, t}^{(k)} \rightarrow\left|\varphi_{t}\right\rangle\left\langle\left.\varphi_{t}\right|^{\otimes k}\right.$ in the weak* topology of $\mathcal{L}_{k}^{1}$, and thus also in the trace-norm topology. This completes the proof of Theorem 3.2

\section{THE WAVE OPERATOR AND A PRIORI BOUNDS ON $\gamma_{N, t}^{(k)}$}

In order to derive a priori bounds for the marginal densities $\gamma_{N, t}^{(k)}$, we need to introduce wave operators. We denote by $W$ and $W_{N}$ the wave operators associated with the one-particle Hamiltonian $\mathfrak{h}=-\Delta+(1 / 2) V(x)$ and, respectively, $\mathfrak{h}_{N}=$ $-\Delta+(1 / 2) V_{N}(x)$, with $V_{N}(x)=N^{2} V(N x)$. The existence of these wave operators and their most important properties are stated in the following proposition (we denote by $s-\lim$ the limit in the strong operator topology). 
Proposition 5.1. Suppose $V \geq 0$, with $V \in L^{1}\left(\mathbb{R}^{3}\right)$. Then:

i) (Existence of the wave operator). The limit

$$
W=s-\lim _{t \rightarrow \infty} e^{i \mathfrak{h} t} e^{i \Delta t}
$$

exists.

ii) (Completeness of the wave operator). $W$ is a unitary operator on $L^{2}\left(\mathbb{R}^{3}\right)$ with

$$
W^{*}=W^{-1}=s-\lim _{t \rightarrow \infty} e^{-i \Delta t} e^{-i \mathfrak{h} t} .
$$

iii) (Intertwining relations). On $D(\mathfrak{h})=D(-\Delta)$, we have

$$
W^{*} \mathfrak{h} W=-\Delta \text {. }
$$

iv) (Yajima's bounds). Suppose moreover that $V(x) \leq C\langle x\rangle^{-\sigma}$ for some $\sigma>5$. Then, for every $1 \leq p \leq \infty, W$ and $W^{*}$ map $L^{p}\left(\mathbb{R}^{3}\right)$ into $L^{p}\left(\mathbb{R}^{3}\right)$, and

$$
\|W\|_{L^{p} \rightarrow L^{p}}<\infty \quad \text { for all } \quad 1 \leq p \leq \infty .
$$

v) (Rescaled wave operator). If $\mathfrak{h}_{N}=-\Delta+(1 / 2) V_{N}(x)$ with $V_{N}(x)=N^{2} V(N x)$, then the limit

$$
W_{N}=s-\lim _{t \rightarrow \infty} e^{i \mathfrak{h}_{N} t} e^{i \Delta t}
$$

exists and defines a unitary operator $W_{N}$ on $L^{2}\left(\mathbb{R}^{3}\right)$ with

$$
W_{N}^{*}=W_{N}^{-1}=s-\lim _{t \rightarrow \infty} e^{-i \Delta t} e^{-i \mathfrak{h}_{N} t} .
$$

The wave operator $W_{N}$ satisfies the intertwining relations

$$
W_{N}^{*} \mathfrak{h}_{N} W_{N}=-\Delta \text {. }
$$

Moreover, the kernel of $W_{N}$ is given by

$$
W_{N}(x ; y)=N^{3} W(N x ; N y) \quad \text { and } \quad W_{N}^{*}(x ; y)=N^{3} W^{*}(N x ; N y) \text {, }
$$

where $W(x ; y)$ and $W^{*}(x ; y)$ denote the kernels of $W$ and $W^{*}$. In particular, it follows that, for every $1 \leq p \leq \infty$, the norms

$$
\left\|W_{N}\right\|_{L^{p} \rightarrow L^{p}}=\|W\|_{L^{p} \rightarrow L^{p}}<\infty \quad \text { and } \quad\left\|W_{N}^{*}\right\|_{L^{p} \rightarrow L^{p}}=\left\|W^{*}\right\|_{L^{p} \rightarrow L^{p}}<\infty
$$

are finite and independent of $N$.

Proof. The proof of i), ii), and iii) can be found in [24]. Part iv) is proven in [30, 29]. Part v) follows by simple scaling arguments.

In the following we will denote by $W_{(i, j)}$ and, respectively, by $W_{N,(i, j)}$, the wave operators $W$ and $W_{N}$ acting only on the relative variable $x_{j}-x_{i}$. In other words, the action of $W_{(i, j)}$ on an $N$-particle wave function $\psi_{N} \in L^{2}\left(\mathbb{R}^{3 N}\right)$ is given by

$$
\begin{aligned}
\left(W_{(i, j)} \psi_{N}\right)(\mathbf{x})=\int & \mathrm{d} v W\left(x_{j}-x_{i} ; v\right) \\
& \times \psi_{N}\left(x_{1}, \ldots, \frac{x_{i}+x_{j}}{2}+\frac{v}{2}, \ldots, \frac{x_{i}+x_{j}}{2}-\frac{v}{2}, \ldots, x_{N}\right)
\end{aligned}
$$

if $j<i$ (the formula for $i>j$ is similar). Here $W(x ; y)$ is the kernel of the wave operator $W$. An analogous formula holds for the rescaled wave operator $W_{N}$. Similarly, we define $W_{(i, j)}^{*}$ and $W_{N,(i, j)}^{*}$.

Using the wave operators, we have the following energy estimate. 
Proposition 5.2. Suppose $V \geq 0, V \in L^{1}\left(\mathbb{R}^{3}\right)$ and $V(x)=V(-x)$ for all $x \in \mathbb{R}^{3}$. Then we have, for every $i \neq j$,

$$
\left\langle\psi_{N}, H_{N}^{2} \psi_{N}\right\rangle \geq C N^{2} \int \mathrm{d} \mathbf{x}\left|\left(\nabla_{i} \cdot \nabla_{j}\right)^{2} W_{N,(i, j)}^{*} \psi_{N}\right|^{2}
$$

where $W_{N,(i, j)}^{*}$ denotes the wave operator $W_{N}^{*}$ defined in Proposition 5.1 acting on the variable $v=x_{j}-x_{i}$ (defined similarly to (5.2)).

Proof. We define, for $j=1, \ldots, N$,

$$
h_{j}=-\Delta_{j}+\frac{1}{2} \sum_{i \neq j} V_{N}\left(x_{i}-x_{j}\right) .
$$

Then we have $H_{N}=\sum_{j=1}^{N} h_{j}$, and thus

$$
\begin{aligned}
\left\langle\psi_{N}, H_{N}^{2} \psi_{N}\right\rangle \geq & N(N-1)\left\langle\psi_{N}, h_{1} h_{2} \psi_{N}\right\rangle \\
= & N(N-1)\left\langle\psi_{N},\left(-\Delta_{1}+\frac{1}{2} \sum_{i \neq 1} V_{N}\left(x_{i}-x_{1}\right)\right)\right. \\
\times & \left.\left(-\Delta_{2}+\frac{1}{2} \sum_{j \neq 2} V_{N}\left(x_{j}-x_{2}\right)\right) \psi_{N}\right\rangle \\
\geq N(N-1)\left\langle\psi_{N},\right. & \left(-\Delta_{1}+\frac{1}{2} V_{N}\left(x_{1}-x_{2}\right)\right) \\
& \left.\times\left(-\Delta_{2}+\frac{1}{2} V_{N}\left(x_{1}-x_{2}\right)\right) \psi_{N}\right\rangle .
\end{aligned}
$$

Now we define the new variables

$$
u=\frac{x_{1}+x_{2}}{2} \text { and } \quad v=x_{1}-x_{2} .
$$

Then we have

$$
\nabla_{1}=\frac{1}{2} \nabla_{u}+\nabla_{v} \quad \text { and } \quad \nabla_{2}=\frac{1}{2} \nabla_{u}-\nabla_{v}
$$

and thus

$$
\Delta_{1}=\frac{1}{4} \Delta_{u}+\Delta_{v}+\nabla_{u} \cdot \nabla_{v} \quad \text { and } \quad \Delta_{2}=\frac{1}{4} \Delta_{u}+\Delta_{v}-\nabla_{u} \cdot \nabla_{v} .
$$

We set

$$
h_{v}=-\Delta_{v}+\frac{1}{2} V_{N}(v)
$$

Then

$$
\begin{aligned}
& \left\langle\psi_{N}, H_{N}^{2} \psi_{N}\right\rangle \\
& \quad \geq N(N-1)\left\langle\psi_{N},\left(-\frac{1}{4} \Delta_{u}+h_{v}+\nabla_{u} \cdot \nabla_{v}\right)\left(-\frac{1}{4} \Delta_{u}+h_{v}-\nabla_{u} \cdot \nabla_{v}\right) \psi_{N}\right\rangle \\
& =N(N-1)\left\langle\psi_{N},\left[\left(-\frac{1}{4} \Delta_{u}+h_{v}\right)^{2}-\left(\nabla_{u} \cdot \nabla_{v}\right)^{2}+\frac{1}{2} \nabla_{u} \cdot\left(\nabla V_{N}(v)\right)\right] \psi_{N}\right\rangle .
\end{aligned}
$$


Next we note that

$$
\begin{array}{r}
\left\langle\psi_{N}, \nabla_{u} \cdot \nabla V_{N}(v) \psi_{N}\right\rangle=\int \mathrm{d} u \mathrm{~d} v \bar{\psi}_{N}\left(u+v / 2, u-v / 2, \mathbf{x}_{N-2}\right) \nabla V_{N}(v) \\
\cdot \nabla_{u} \psi_{N}\left(u+v / 2, u-v / 2, \mathbf{x}_{N-2}\right)=0 .
\end{array}
$$

In fact, by the permutation symmetry, $\psi_{N}\left(x_{1}, x_{2}, \mathbf{x}_{N-2}\right)=\psi_{N}\left(x_{2}, x_{1}, \mathbf{x}_{N-2}\right)$. This implies, in the $u, v$-coordinates, that

$$
\psi_{N}\left(u+v / 2, u-v / 2, \mathbf{x}_{N-2}\right)=\psi_{N}\left(u-v / 2, u+v / 2, \mathbf{x}_{N-2}\right)
$$

and also that $\nabla_{u} \psi_{N}\left(u+v / 2, u-v / 2, \mathbf{x}_{N-2}\right)=\nabla_{u} \psi_{N}\left(u-v / 2, u+v / 2, \mathbf{x}_{N-2}\right)$. On the other hand $\left(\nabla V_{N}\right)(-v)=-\left(\nabla V_{N}\right)(v)$. Therefore, the integrand in (5.6) is antisymmetric w.r.t. the change of variables $v \rightarrow-v$, and the integral vanishes.

Also using the fact that

$$
\left(\nabla_{u} \cdot \nabla_{v}\right)^{2} \leq\left(-\Delta_{u}\right)\left(-\Delta_{v}\right) \leq\left(-\Delta_{u}\right) h_{v},
$$

it follows from (5.5) that

$$
\left\langle\psi_{N}, H_{N}^{2} \psi_{N}\right\rangle \geq N(N-1)\left\langle\psi_{N},\left(-\frac{1}{4} \Delta_{u}-h_{v}\right)^{2} \psi_{N}\right\rangle .
$$

Next we make use of the wave operator $W_{N}$ defined in Proposition 5.1, acting on the variable $v=x_{2}-x_{1}$. By the intertwining relations (5.1), we find

$$
\left\langle\psi_{N}, H_{N}^{2} \psi_{N}\right\rangle \geq N(N-1)\left\langle W_{N,(1,2)}^{*} \psi_{N},\left(\frac{1}{4} \Delta_{u}-\Delta_{v}\right)^{2} W_{N,(1,2)}^{*} \psi_{N}\right\rangle .
$$

In terms of the coordinates $x_{1}$ and $x_{2}$, we have $\nabla_{1} \cdot \nabla_{2}=(1 / 4) \Delta_{u}-\Delta_{v}$. Therefore, by the permutation symmetry, the bound (5.8) implies (5.3).

Proposition 5.2 implies strong a priori bounds on the solution of the $N$-particle Schrödinger equation.

Proposition 5.3. Suppose that $V \geq 0, V \in L^{1}\left(\mathbb{R}^{3}\right)$, and $V(-x)=V(x)$ for all $x \in \mathbb{R}^{3}$. Let $\psi_{N, t}$ be the solution of the Schrödinger equation (1.3), with initial data satisfying assumption (3.4) (with $k=2$ ) of Theorem 3.2, and let $\left\{\gamma_{N, t}^{(k)}\right\}_{k=1}^{N}$ be the marginals associated with $\psi_{N, t}$. Then, for every $1 \leq j \leq N$, we have

$$
\left\langle\psi_{N, t},\left(1-\Delta_{j}\right) \psi_{N, t}\right\rangle \leq C, \quad \text { and thus } \quad \operatorname{Tr}\left(1-\Delta_{j}\right) \gamma_{N, t}^{(k)} \leq C
$$

for every $1 \leq j \leq k \leq N$ (and for a constant $C$ which only depends on the initial data $\psi_{N}$ through the constant on the r.h.s. of (3.4)). Moreover, for any $i \neq j$,

$$
\left\langle W_{N,(i, j)}^{*} \psi_{N, t},\left(\left(\nabla_{i} \cdot \nabla_{j}\right)^{2}-\Delta_{i}-\Delta_{j}+1\right) W_{N,(i, j)}^{*} \psi_{N, t}\right\rangle \leq C
$$

uniformly in $N \geq 1$ and in $t \in \mathbb{R}$. Here $W_{N,(i, j)}$ denotes the wave operator $W_{N}$ defined in Proposition 5.1 acting on the variable $x_{j}-x_{i}$. In terms of density matrices, we obtain the a priori bounds

$$
\operatorname{Tr}\left(\left(\nabla_{i} \cdot \nabla_{j}\right)^{2}-\Delta_{i}-\Delta_{j}+1\right) W_{N,(i, j)}^{*} \gamma_{N, t}^{(k)} W_{N,(i, j)} \leq C
$$

uniformly in $N \geq 1$ and in $t \in \mathbb{R}$ and for all $1 \leq i<j \leq k$ (with a slight abuse of notation, we denote here by $W_{N,(i, j)}$ and $W_{N,(i, j)}^{*}$ the operators acting on the $k$-particle space $L^{2}\left(\mathbb{R}^{3 k}\right)$ ). 
Proof. The first bound follows simply by the symmetry of the wave function, by energy conservation, and by the condition $V \geq 0$. To prove the second bound, we compute

$$
\begin{aligned}
& \left\langle W_{N,(i, j)}^{*} \psi_{N, t},\left(\left(\nabla_{i} \cdot \nabla_{j}\right)^{2}-\Delta_{i}-\Delta_{j}+1\right) W_{N,(i, j)}^{*} \psi_{N, t}\right\rangle \\
& =\left\langle W_{N,(1,2)}^{*} \psi_{N, t},\left(\nabla_{1} \cdot \nabla_{2}\right)^{2} W_{N,(1,2)}^{*} \psi_{N, t}\right\rangle \\
& \quad+\left\langle W_{N,(1,2)}^{*} \psi_{N, t},\left(-\Delta_{1}-\Delta_{2}\right) W_{N,(1,2)}^{*} \psi_{N, t}\right\rangle+1 .
\end{aligned}
$$

The first term on the r.h.s. of the last equation can be bounded by

$$
\begin{aligned}
\left\langle W_{N,(1,2)}^{*} \psi_{N, t},\left(\nabla_{1} \cdot \nabla_{2}\right)^{2} W_{N,(1,2)}^{*} \psi_{N, t}\right\rangle & \leq C N^{-2}\left\langle\psi_{N, t}, H_{N}^{2} \psi_{N, t}\right\rangle \\
& =C N^{-2}\left\langle\psi_{N, 0}, H_{N}^{2} \psi_{N, 0}\right\rangle \leq C
\end{aligned}
$$

using Proposition 5.2 and (3.4). The second term on the r.h.s. of (5.9) is estimated by

$$
\begin{aligned}
\left\langle W_{N,(1,2)}^{*} \psi_{N, t},\right. & \left.\left(-\Delta_{1}-\Delta_{2}\right) W_{N,(1,2)}^{*} \psi_{N, t}\right\rangle \\
& =2\left\langle W_{N,(1,2)}^{*} \psi_{N, t},\left(-\Delta_{x_{1}-x_{2}}-\frac{1}{4} \Delta_{\left(x_{1}+x_{2}\right) / 2}\right) W_{N,(1,2)}^{*} \psi_{N, t}\right\rangle \\
& =2\left\langle\psi_{N, t},\left(-\Delta_{x_{1}-x_{2}}+\frac{1}{2} V_{N}\left(x_{1}-x_{2}\right)-\frac{1}{4} \Delta_{\left(x_{1}+x_{2}\right) / 2}\right) \psi_{N, t}\right\rangle \\
& =\left\langle\psi_{N, t},\left(-\Delta_{x_{1}}-\Delta_{x_{2}}+V_{N}\left(x_{1}-x_{2}\right)\right) \psi_{N, t}\right\rangle \\
& \leq \frac{2}{N}\left\langle\psi_{N, t}, H_{N} \psi_{N, t}\right\rangle=\frac{2}{N}\left\langle\psi_{N, 0}, H_{N} \psi_{N, 0}\right\rangle \leq C .
\end{aligned}
$$

\section{Compactness}

In this section we prove the compactness of the sequence $\Gamma_{N, t}=\left\{\gamma_{N, t}^{(k)}\right\}_{k \geq 1}$ w.r.t. the topology $\tau_{\text {prod }}$ (defined in Section 4).

Theorem 6.1. Let the assumptions of Theorem 3.2 be satisfied and fix an arbitrary $T>0$. Then the sequence $\Gamma_{N, t} \in \bigoplus_{k \geq 1} C\left([0, T], \mathcal{L}_{k}^{1}\right)$ is compact with respect to the product topology $\tau_{\text {prod }}$ generated by the metrics $\widehat{\eta}_{k}$ (defined in Section 4 ). For any limit point $\Gamma_{\infty, t}=\left\{\gamma_{\infty, t}^{(k)}\right\}_{k \geq 1}, \gamma_{\infty, t}^{(k)}$ is symmetric w.r.t. permutations, $\gamma_{\infty, t}^{(k)} \geq 0$, and

$$
\operatorname{Tr} \gamma_{\infty, t}^{(k)} \leq 1
$$

for every $k \geq 1$.

Proof. By a standard argument it is enough to prove the compactness of $\gamma_{N, t}^{(k)}$ for fixed $k \geq 1$ with respect to the metric $\widehat{\eta}_{k}$. To this end, it is enough to show the equicontinuity of $\gamma_{N, t}^{(k)}$ with respect to the metric $\eta_{k}$. A useful criterium for equicontinuity is given by the following lemma, whose proof can be found in [11, Proposition 9.2].

Lemma 6.2. Fix $k \in \mathbb{N}$ and $T>0$. A sequence $\gamma_{N, t}^{(k)} \in \mathcal{L}_{k}^{1}, N=k, k+1, \ldots$, with $\gamma_{N, t}^{(k)} \geq 0$ and $\operatorname{Tr} \gamma_{N, t}^{(k)}=1$ for all $t \in[0, T]$ and $N \geq k$, is equicontinuous in $C\left([0, T], \mathcal{L}_{k}^{1}\right)$ with respect to the metric $\eta_{k}$, if and only if there exists a dense subset 
$\mathcal{J}_{k}$ of $\mathcal{K}_{k}$ such that for any $J^{(k)} \in \mathcal{J}_{k}$ and for every $\varepsilon>0$ there exists a $\delta>0$ such that

$$
\sup _{N \geq 1}\left|\operatorname{Tr} J^{(k)}\left(\gamma_{N, t}^{(k)}-\gamma_{N, s}^{(k)}\right)\right| \leq \varepsilon
$$

for all $t, s \in[0, T]$ with $|t-s| \leq \delta$.

We prove (6.2) for all $J^{(k)} \in \mathcal{K}_{k}$ such that $\left\|J^{(k)}\right\|<\infty$, where we introduced the norm

$$
\begin{aligned}
\left\|J^{(k)}\right\|=\sup _{\mathbf{x}_{k}, \mathbf{x}_{k}^{\prime}} & \left\langle x_{1}\right\rangle^{4} \ldots\left\langle x_{k}\right\rangle^{4}\left\langle x_{1}^{\prime}\right\rangle^{4} \ldots\left\langle x_{k}^{\prime}\right\rangle^{4} \\
& \times\left(\left|J^{(k)}\left(\mathbf{x}_{k}^{\prime} ; \mathbf{x}_{k}\right)\right|+\sum_{j=1}^{k}\left(\left|\nabla_{x_{j}} J^{(k)}\left(\mathbf{x}_{k}^{\prime} ; \mathbf{x}_{k}\right)\right|+\left|\nabla_{x_{j}^{\prime}} J^{(k)}\left(\mathbf{x}_{k}^{\prime} ; \mathbf{x}_{k}\right)\right|\right)\right) .
\end{aligned}
$$

It is simple to check that the set of $J^{(k)} \in \mathcal{K}_{k}$ for which $\left\|J^{(k)}\right\|<\infty$ is dense in $\mathcal{K}_{k}$.

Rewriting the BBGKY hierarchy (2.1) in integral form and multiplying with an arbitrary observable $J^{(k)} \in \mathcal{K}_{k}$ with $\left\|J^{(k)}\right\|<\infty$, we obtain that, for any $r \leq t$,

$$
\begin{aligned}
\left|\operatorname{Tr} J^{(k)}\left(\gamma_{N, t}^{(k)}-\gamma_{N, r}^{(k)}\right)\right| \leq & \sum_{j=1}^{k} \int_{r}^{t} \mathrm{~d} s\left|\operatorname{Tr} J^{(k)}\left[-\Delta_{j}, \gamma_{N, s}^{(k)}\right]\right| \\
& +\sum_{i<j}^{k} \int_{r}^{t} \mathrm{~d} s\left|\operatorname{Tr} J^{(k)}\left[V_{N}\left(x_{i}-x_{j}\right), \gamma_{N, s}^{(k)}\right]\right| \\
& +\left(1-\frac{k}{N}\right) \sum_{j=1}^{k} \int_{r}^{t} \mathrm{~d} s\left|\operatorname{Tr} J^{(k)}\left[N V_{N}\left(x_{j}-x_{k+1}\right), \gamma_{N, s}^{(k+1)}\right]\right|
\end{aligned}
$$

To control the first term on the r.h.s. of the last equation, we observe that, using the notation $S_{j}=\left(1-\Delta_{j}\right)^{1 / 2}$, we have

$$
\begin{aligned}
\left|\operatorname{Tr} J^{(k)}\left[-\Delta_{j}, \gamma_{N, s}^{(k)}\right]\right| & =\left|\operatorname{Tr}\left(S_{j}^{-1} J^{(k)} S_{j}-S_{j} J^{(k)} S_{j}^{-1}\right) S_{j} \gamma_{N, s}^{(k)} S_{j}\right| \\
& \leq\left(\left\|S_{j}^{-1} J^{(k)} S_{j}\right\|+\left\|S_{j} J^{(k)} S_{j}^{-1}\right\|\right) \operatorname{Tr}\left(1-\Delta_{j}\right) \gamma_{N, s}^{(k)} \\
& \leq C\left\|J^{(k)}\right\| .
\end{aligned}
$$

Here we used the fact that, by Proposition 5.3 .

$$
\sup _{s \in \mathbb{R}} \operatorname{Tr}\left(1-\Delta_{j}\right) \gamma_{N, s}^{(k)} \leq C
$$

uniformly in $N$.

To bound the second term on the r.h.s. of (6.4), we decompose

$$
\gamma_{N, s}^{(k)}=\sum_{\ell} \lambda_{\ell}^{(k)}\left|\xi_{\ell}^{(k)}\right\rangle\left\langle\xi_{\ell}^{(k)}\right|
$$

for $\xi_{\ell}^{(k)} \in L^{2}\left(\mathbb{R}^{3 k}\right)$, with $\left\|\xi_{\ell}^{(k)}\right\|=1, \lambda_{\ell}^{(k)}>0$, and $\sum_{\ell} \lambda_{\ell}^{(k)}=1$ (here we omitted the dependence of $\xi_{\ell}^{(k)}$ and $\lambda_{\ell}^{(k)}$ on $N, s$ from the notation). Then we find, for example 
for the term with $i=1, j=2$,

$$
\begin{aligned}
& \operatorname{Tr} J^{(k)} V_{N}\left(x_{1}-x_{2}\right) \gamma_{N, s}^{(k)} \\
& \quad=\sum_{\ell} \lambda_{\ell}^{(k)} \int \mathrm{d} \mathbf{x}_{k} \mathrm{~d} \mathbf{x}_{k}^{\prime} J^{(k)}\left(\mathbf{x}_{k}^{\prime} ; \mathbf{x}_{k}\right) V_{N}\left(x_{1}-x_{2}\right) \xi_{\ell}^{(k)}\left(\mathbf{x}_{k}\right) \bar{\xi}_{\ell}^{(k)}\left(\mathbf{x}_{k}^{\prime}\right) .
\end{aligned}
$$

Denoting by $W_{N}$ the wave operator associated with the Hamiltonian $\mathfrak{h}_{N}=-\Delta+$ $(1 / 2) V_{N}(x)$, and by $W_{N,(i, j)}$ the wave operator $W_{N}$ acting on the variable $x_{j}-x_{i}$ (as defined in (5.2) ), we can estimate (introducing the new variables $u=\left(x_{1}+x_{2}\right) / 2$ and $\left.v=x_{1}-x_{2}\right)$

$$
\begin{array}{r}
\left|\int \mathrm{d} \mathbf{x}_{k} \mathrm{~d} \mathbf{x}_{k}^{\prime} J^{(k)}\left(\mathbf{x}_{k}^{\prime} ; \mathbf{x}_{k}\right) V_{N}\left(x_{1}-x_{2}\right) \xi_{\ell}^{(k)}\left(\mathbf{x}_{k}\right) \bar{\xi}_{\ell}^{(k)}\left(\mathbf{x}_{k}^{\prime}\right)\right| \\
=\mid \int \mathrm{d} u \mathrm{~d} v \mathrm{~d} x_{3} \ldots \mathrm{d} x_{k} \mathrm{~d} \mathbf{x}_{k}^{\prime} J^{(k)}\left(\mathbf{x}_{k}^{\prime} ; u+v / 2, u-v / 2, x_{3}, \ldots, x_{k}\right) \\
\times V_{N}(v) \xi_{\ell}^{(k)}\left(u+v / 2, u-v / 2, x_{3}, \ldots, x_{k}\right) \bar{\xi}_{\ell}^{(k)}\left(\mathbf{x}_{k}^{\prime}\right) \mid \\
\leq \int \mathrm{d} u \mathrm{~d} v \mathrm{~d} x_{3} \ldots \mathrm{d} x_{k} \mathrm{~d} \mathbf{x}_{k}^{\prime} \mid J^{(k)}\left(\mathbf{x}_{k}^{\prime} ; u+v / 2, u-v / 2, x_{3}, \ldots, x_{k}\right) \\
-J^{(k)}\left(\mathbf{x}_{k}^{\prime} ; u, u, x_{3}, \ldots, x_{k}\right) \mid \\
\times V_{N}(v)\left|\xi_{\ell}^{(k)}\left(u+v / 2, u-v / 2, x_{3}, \ldots, x_{k}\right)\right|\left|\xi_{\ell}^{(k)}\left(\mathbf{x}_{k}^{\prime}\right)\right| \\
+\int \mathrm{d} u \mathrm{~d} v \mathrm{~d} x_{3} \ldots \mathrm{d} x_{k} \mathrm{~d} \mathbf{x}_{k}^{\prime}\left|J^{(k)}\left(\mathbf{x}_{k}^{\prime} ; u, u, x_{3}, \ldots, x_{k}\right)\right|\left|\left(W_{N}^{*} V_{N}\right)(v)\right| \\
\times\left|\left(W_{N,(1,2)}^{*} \xi_{\ell}^{(k)}\right)\left(u+v / 2, u-v / 2, x_{3}, \ldots, x_{k}\right)\right|\left|\xi_{\ell}^{(k)}\left(\mathbf{x}_{k}^{\prime}\right)\right|,
\end{array}
$$

where in the last line we used the $L^{2}$-unitarity of the wave operator in the $v$-variable (before taking the absolute value inside the integral). Hence

$$
\begin{aligned}
& \left|\int \mathrm{d} \mathbf{x}_{k} \mathrm{~d} \mathbf{x}_{k}^{\prime} J^{(k)}\left(\mathbf{x}_{k}^{\prime} ; \mathbf{x}_{k}\right) V_{N}\left(x_{1}-x_{2}\right) \xi_{\ell}^{(k)}\left(\mathbf{x}_{k}\right) \bar{\xi}_{\ell}^{(k)}\left(\mathbf{x}_{k}^{\prime}\right)\right| \\
& \leq \sum_{j=1}^{2} \int \mathrm{d} u \mathrm{~d} v \mathrm{~d} x_{3} \ldots \mathrm{d} x_{k} \mathrm{~d} \mathbf{x}_{k}^{\prime} \int_{0}^{1} \mathrm{~d} \tau\left|\nabla_{x_{j}} J^{(k)}\left(\mathbf{x}_{k}^{\prime} ; u+\frac{\tau v}{2}, u-\frac{\tau v}{2}, x_{3}, \ldots, x_{k}\right)\right| \\
& \times|v| V_{N}(v)\left|\xi_{\ell}^{(k)}\left(\mathbf{x}_{k}^{\prime}\right)\right|^{2} \\
& +\sum_{j=1}^{2} \int \mathrm{d} u \mathrm{~d} v \mathrm{~d} x_{3} \ldots \mathrm{d} x_{k} \mathrm{~d} \mathbf{x}_{k}^{\prime} \int_{0}^{1} \mathrm{~d} \tau\left|\nabla_{x_{j}} J^{(k)}\left(\mathbf{x}_{k}^{\prime} ; u+\frac{\tau v}{2}, u-\frac{\tau v}{2}, x_{3}, \ldots, x_{k}\right)\right| \\
& \times|v| V_{N}(v)\left|\xi_{\ell}^{(k)}\left(u+v / 2, u-v / 2, x_{3}, \ldots, x_{k}\right)\right|^{2} \\
& +\int \mathrm{d} u \mathrm{~d} v \mathrm{~d} x_{3} \ldots \mathrm{d} x_{k} \mathrm{~d} \mathbf{x}_{k}^{\prime}\left|J^{(k)}\left(\mathbf{x}_{k}^{\prime} ; u, u, x_{3}, \ldots, x_{k}\right)\right|\left|\left(W_{N}^{*} V_{N}\right)(v)\right|\left|\xi_{\ell}^{(k)}\left(\mathbf{x}_{k}^{\prime}\right)\right|^{2} \\
& +\int \mathrm{d} u \mathrm{~d} v \mathrm{~d} x_{3} \ldots \mathrm{d} x_{k} \mathrm{~d} \mathbf{x}_{k}^{\prime}\left|J^{(k)}\left(\mathbf{x}_{k}^{\prime} ; u, u, x_{3}, \ldots, x_{k}\right)\right|\left|\left(W_{N}^{*} V_{N}\right)(v)\right| \\
& \times\left|\left(W_{N,(1,2)}^{*} \xi_{\ell}^{(k)}\right)\left(u+v / 2, u-v / 2, x_{3}, \ldots, x_{k}\right)\right|^{2} .
\end{aligned}
$$


Using the norm defined in (6.3), we find

$$
\begin{aligned}
& \left|\int \mathrm{d} \mathbf{x}_{k} \mathrm{~d} \mathbf{x}_{k}^{\prime} J^{(k)}\left(\mathbf{x}_{k}^{\prime} ; \mathbf{x}_{k}\right) V_{N}\left(x_{1}-x_{2}\right) \xi_{\ell}^{(k)}\left(\mathbf{x}_{k}\right) \bar{\xi}_{\ell}^{(k)}\left(\mathbf{x}_{k}^{\prime}\right)\right| \\
& \leq C_{k}\left\|J^{(k)}\right\|\left\|\xi_{\ell}^{(k)}\right\|^{2} \int \mathrm{d} v|v| V_{N}(v) \\
& \quad+C_{k}\left\|J^{(k)}\right\| \int \mathrm{d} v \mathrm{~d} u \mathrm{~d} x_{3} \ldots \mathrm{d} x_{N}|v| V_{N}(v)\left|\xi_{\ell}^{(k)}\left(u+v / 2, u-v / 2, x_{3}, \ldots, x_{k}\right)\right|^{2} \\
& \quad+C_{k}\left\|J^{(k)}\right\|\left\|\xi_{\ell}^{(k)}\right\|^{2} \int \mathrm{d} v\left|\left(W_{N}^{*} V_{N}\right)(v)\right| \\
& \quad+C_{k}\left\|J^{(k)}\right\| \int \mathrm{d} u \mathrm{~d} v \mathrm{~d} x_{3} \ldots \mathrm{d} x_{k}\left|\left(W_{N}^{*} V_{N}\right)(v)\right| \\
& \quad \times\left|\left(W_{N,(1,2)}^{*} \xi_{\ell}^{(k)}\right)\left(u+v / 2, u-v / 2, x_{3}, \ldots, x_{k}\right)\right|^{2} .
\end{aligned}
$$

Since, by scaling $\left\||v| V_{N}\right\|_{1} \leq C N^{-2}$ and $\left\||v| V_{N}\right\|_{3 / 2} \leq C N^{-1}$ and since $\left\|W_{N}^{*} V_{N}\right\|_{1}$ $\leq C\left\|V_{N}\right\|_{1} \leq C N^{-1}$ (by the Yajima's bounds in part v) of Proposition [5.1), we conclude that

$$
\begin{aligned}
& \left|\int \mathrm{d} \mathbf{x}_{k} \mathrm{~d} \mathbf{x}_{k}^{\prime} J^{(k)}\left(\mathbf{x}_{k}^{\prime} ; \mathbf{x}_{k}\right) V_{N}\left(x_{1}-x_{2}\right) \xi_{\ell}^{(k)}\left(\mathbf{x}_{k}\right) \bar{\xi}_{\ell}^{(k)}\left(\mathbf{x}_{k}^{\prime}\right)\right| \\
& \quad \leq \frac{C_{k}\left\|J^{(k)}\right\|}{N}\left\langle W_{N,(1,2)}^{*} \xi_{\ell}^{(k)},\left(\left(\nabla_{1} \cdot \nabla_{2}\right)^{2}-\Delta_{1}-\Delta_{2}+1\right) W_{N,(1,2)}^{*} \xi_{\ell}^{(k)}\right\rangle
\end{aligned}
$$

for a $k$-dependent constant $C_{k}$. Here we used Lemma 10.1 to bound the last term on the r.h.s. of (6.7), and, by the Sobolev inequality $\left\|\xi_{\ell}^{(k)}\right\|_{L_{v}^{6}} \leq C\left\|\nabla_{v} \xi_{\ell}^{(k)}\right\|_{L_{v}^{2}}$ we used

$$
\begin{aligned}
\int \mathrm{d} u & \mathrm{~d} x_{3} \ldots \mathrm{d} x_{k} \mathrm{~d} v|v| V_{N}(v)\left|\xi_{\ell}^{(k)}\left(u+v / 2, u-v / 2, x_{3}, \ldots, x_{k}\right)\right|^{2} \\
& \leq\left\||v| V_{N}\right\|_{3 / 2} \int \mathrm{d} u \mathrm{~d} x_{3} \ldots \mathrm{d} x_{k} \mathrm{~d} v\left|\nabla_{v} \xi_{\ell}^{(k)}\left(u+\frac{v}{2}, u-\frac{v}{2}, x_{3}, \ldots, x_{k}\right)\right|^{2} \\
& \leq C N^{-1}\left\langle\xi_{\ell}^{(k)},\left(-\Delta_{v}\right) \xi_{\ell}^{(k)}\right\rangle \\
& \leq C N^{-1}\left\langle\xi_{\ell}^{(k)},\left(-\Delta_{v}+(1 / 2) V_{N}(v)\right) \xi_{\ell}^{(k)}\right\rangle \\
& \leq C N^{-1}\left\langle W_{N,(1,2)}^{*} \xi_{\ell}^{(k)},\left(-\Delta_{1}-\Delta_{2}\right) W_{N,(1,2)}^{*} \xi_{\ell}^{(k)}\right\rangle
\end{aligned}
$$

to bound the second term on the r.h.s. of (6.7) (applying the intertwining relations (5.1) and adding a positive term $-\Delta_{u}$ as in (5.11)). From (6.6), (6.8), and from Proposition 5.3 we obtain that

$$
\begin{aligned}
& \left|\operatorname{Tr} J^{(k)} V_{N}\left(x_{1}-x_{2}\right) \gamma_{N, s}^{(k)}\right| \\
& \quad \leq \frac{C_{k}\left\|J^{(k)}\right\|}{N} \operatorname{Tr}\left(\left(\nabla_{1} \cdot \nabla_{2}\right)^{2}-\Delta_{1}-\Delta_{2}+1\right) W_{N,(1,2)}^{*} \gamma_{N, s}^{(k)} W_{N,(1,2)} \\
& \quad \leq \frac{C_{k}\left\|J^{(k)}\right\|}{N}
\end{aligned}
$$


for all $s \in \mathbb{R}$ and a constant $C_{k}$ only depending on $k$ (and on the constant appearing on the r.h.s. of (3.4) ). Similarly to (6.10), we can also show that

$$
\left|\operatorname{Tr} J^{(k)} \gamma_{N, s}^{(k)} V_{N}\left(x_{1}-x_{2}\right)\right| \leq \frac{C_{k}\left\|J^{(k)}\right\|}{N}
$$

Since (6.10) and (6.11) remain valid for all summands in the second term on the r.h.s. of (6.4), we obtain that, for all $k \in \mathbb{N}$, for all $t \in[0, T]$ and for all $J^{(k)} \in \mathcal{K}_{k}$ with $\left\|J^{(k)}\right\|<\infty$,

$$
\sum_{i<j}^{k}\left|\operatorname{Tr} J^{(k)}\left[V_{N}\left(x_{i}-x_{j}\right), \gamma_{N, s}^{(k)}\right]\right| \leq \frac{C_{k}\left\|J^{(k)}\right\|}{N}
$$

for all $s \in \mathbb{R}$.

Also the third term on the r.h.s. of (6.4) can be bounded similarly. In fact, again using the decomposition $\gamma_{N, s}^{(k+1)}=\sum_{\ell} \lambda_{\ell}^{(k+1)}\left|\xi_{\ell}^{(k+1)}\right\rangle\left\langle\xi_{\ell}^{(k+1)}\right|$ we have, for example considering the term with $j=1$,

$$
\begin{aligned}
& \operatorname{Tr} J^{(k)} N V_{N}\left(x_{1}-x_{k+1}\right) \gamma_{N, s}^{(k+1)} \\
& =\sum_{\ell} \lambda_{\ell}^{(k+1)} \int \mathrm{d} \mathbf{x}_{k} \mathrm{~d} \mathbf{x}_{k}^{\prime} \mathrm{d} x_{k+1} J^{(k)}\left(\mathbf{x}_{k}^{\prime} ; \mathbf{x}_{k}\right) N V_{N}\left(x_{1}-x_{k+1}\right) \\
& \quad \times \xi_{\ell}^{(k+1)}\left(\mathbf{x}_{k}, x_{k+1}\right) \bar{\xi}_{\ell}^{(k+1)}\left(\mathbf{x}_{k}^{\prime}, x_{k+1}\right) .
\end{aligned}
$$

The absolute value of the $\ell$-th summand can be estimated by

$$
\begin{aligned}
& \left|\int \mathrm{d} \mathbf{x}_{k} \mathrm{~d} \mathbf{x}_{k}^{\prime} \mathrm{d} x_{k+1} J^{(k)}\left(\mathbf{x}_{k}^{\prime} ; \mathbf{x}_{k}\right) N V_{N}\left(x_{1}-x_{k+1}\right) \xi_{\ell}^{(k+1)}\left(\mathbf{x}_{k}, x_{k+1}\right) \bar{\xi}_{\ell}^{(k+1)}\left(\mathbf{x}_{k}^{\prime}, x_{k+1}\right)\right| \\
& \leq \int \mathrm{d} u \mathrm{~d} v \mathrm{~d} x_{2} \ldots \mathrm{d} x_{k} \mathrm{~d} \mathbf{x}_{k}^{\prime} N V_{N}(v)\left|\xi_{\ell}^{(k+1)}\left(u+v / 2, x_{2}, \ldots, x_{k}, u-v / 2\right)\right| \\
& \times \mid J^{(k)}\left(\mathbf{x}_{k}^{\prime} ; u+v / 2, x_{2}, \ldots, x_{k}\right) \bar{\xi}_{\ell}^{(k+1)}\left(\mathbf{x}_{k}^{\prime}, u-v / 2\right) \\
& -J^{(k)}\left(\mathbf{x}_{k}^{\prime} ; u, x_{2}, \ldots, x_{k}\right) \bar{\xi}_{\ell}^{(k+1)}\left(\mathbf{x}_{k}^{\prime}, u\right) \mid \\
& +\int \mathrm{d} u \mathrm{~d} v \mathrm{~d} x_{2} \ldots \mathrm{d} x_{k} \mathrm{~d} \mathbf{x}_{k}^{\prime} N\left|\left(W_{N}^{*} V_{N}\right)(v)\right|\left|J^{(k)}\left(\mathbf{x}_{k}^{\prime} ; u, x_{2}, \ldots, x_{k}\right)\right| \\
& \quad \times\left|\xi_{\ell}^{(k+1)}\left(\mathbf{x}_{k}^{\prime}, u\right)\right|\left|\left(W_{N,(1, k+1)}^{*} \xi_{\ell}^{(k+1)}\right)\left(u+v / 2, x_{2}, \ldots, x_{k}, u-v / 2\right)\right| \\
& =\mathrm{I}+\mathrm{II} .
\end{aligned}
$$


Here

$$
\begin{aligned}
& \mathrm{I}=\int \mathrm{d} u \mathrm{~d} v \mathrm{~d} x_{2} \ldots \mathrm{d} x_{k} \mathrm{~d} \mathbf{x}_{k}^{\prime} N V_{N}(v)\left|\xi_{\ell}^{(k+1)}\left(u+v / 2, x_{2}, \ldots, x_{k}, u-v / 2\right)\right| \\
& \times\left|\int_{0}^{1} \mathrm{~d} \tau \frac{\mathrm{d}}{\mathrm{d} \tau}\left[J^{(k)}\left(\mathbf{x}_{k}^{\prime} ; u+\frac{\tau v}{2}, x_{2}, \ldots, x_{k}\right) \bar{\xi}_{\ell}^{(k+1)}\left(\mathbf{x}_{k}^{\prime}, u-\frac{\tau v}{2}\right)\right]\right| \\
& \leq \int \mathrm{d} u \mathrm{~d} v \mathrm{~d} x_{2} \ldots \mathrm{d} x_{k} \mathrm{~d} \mathbf{x}_{k}^{\prime} \int_{0}^{1} \mathrm{~d} \tau N V_{N}(v)|v|\left|\xi_{\ell}^{(k+1)}\left(u+v / 2, x_{2}, \ldots, x_{k}, u-v / 2\right)\right| \\
& \times\left(\left|\nabla_{x_{1}} J^{(k)}\left(\mathbf{x}_{k}^{\prime} ; u+\frac{\tau v}{2}, x_{2}, \ldots, x_{k}\right)\right|\left|\xi_{\ell}^{(k+1)}\left(\mathbf{x}_{k}^{\prime}, u-\frac{\tau v}{2}\right)\right|\right. \\
& \left.\quad+\left|J^{(k)}\left(\mathbf{x}_{k}^{\prime} ; u+\frac{\tau v}{2}, x_{2}, \ldots, x_{k}\right)\right|\left|\nabla_{x_{k+1}} \xi_{\ell}^{(k+1)}\left(\mathbf{x}_{k}^{\prime}, u-\frac{\tau v}{2}\right)\right|\right) .
\end{aligned}
$$

Through a weighted Schwarz inequality, we find

$$
\begin{array}{r}
\mathrm{I} \leq \int \mathrm{d} u \mathrm{~d} v \mathrm{~d} x_{2} \ldots \mathrm{d} x_{k} \mathrm{~d} \mathbf{x}_{k}^{\prime} \int_{0}^{1} \mathrm{~d} \tau N V_{N}(v)|v|\left|\nabla_{x_{1}} J^{(k)}\left(\mathbf{x}_{k}^{\prime} ; u+\frac{\tau v}{2}, x_{2}, \ldots, x_{k}\right)\right| \\
\times\left(N^{-1 / 2}\left|\xi_{\ell}^{(k+1)}\left(u+v / 2, x_{2}, \ldots, x_{k}, u-v / 2\right)\right|^{2}\right. \\
\left.+N^{1 / 2}\left|\xi_{\ell}^{(k+1)}\left(\mathbf{x}_{k}^{\prime}, u-\frac{\tau v}{2}\right)\right|^{2}\right) \\
+\int \mathrm{d} u \mathrm{~d} v \mathrm{~d} x_{2} \ldots \mathrm{d} x_{k} \mathrm{~d} \mathbf{x}_{k}^{\prime} \int_{0}^{1} \mathrm{~d} \tau N V_{N}(v)|v|\left|J^{(k)}\left(\mathbf{x}_{k}^{\prime} ; u+\frac{\tau v}{2}, x_{2}, \ldots, x_{k}\right)\right| \\
\times\left(N^{-1 / 2}\left|\xi_{\ell}^{(k+1)}\left(u+v / 2, x_{2}, \ldots, x_{k}, u-v / 2\right)\right|^{2}\right. \\
\left.+N^{1 / 2}\left|\nabla_{x_{k+1}} \xi_{\ell}^{(k+1)}\left(\mathbf{x}_{k}^{\prime}, u-\frac{\tau v}{2}\right)\right|^{2}\right) .
\end{array}
$$

Extracting the observable from the integral (after integrating some of its variables and taking the supremum over the other), and using Sobolev inequalities where needed, we find

$$
\begin{aligned}
\mathrm{I} \leq & C_{k} N^{1 / 2}\left\||v| V_{N}\right\|_{3 / 2}\left\|J^{(k)}\right\| \\
& \times \int \mathrm{d} u \mathrm{~d} v \mathrm{~d} x_{2} \ldots \mathrm{d} x_{k}\left|\nabla_{v} \xi_{\ell}^{(k+1)}\left(u+v / 2, x_{2}, \ldots, x_{k}, u-v / 2\right)\right|^{2} \\
& +C_{k} N^{3 / 2}\left\||v| V_{N}\right\|_{1}\left\|J^{(k)}\right\|\left(\left\|\xi_{\ell}^{(k)}\right\|^{2}+\int \mathrm{d} u \mathrm{~d} \mathbf{x}_{k}^{\prime}\left|\nabla_{k+1} \xi_{\ell}^{(k+1)}\left(\mathbf{x}_{k}^{\prime}, u\right)\right|^{2}\right) \\
\leq & \frac{C_{k}\left\|J^{(k)}\right\|}{N^{1 / 2}}\left\langle W_{N,(1, k+1)}^{*} \xi_{\ell}^{(k+1)},\left(-\Delta_{1}-\Delta_{k+1}+1\right) W_{N,(1, k+1)}^{*} \xi_{\ell}^{(k+1)}\right\rangle .
\end{aligned}
$$

In the last line we proceeded similarly to (6.9) for the two terms with derivatives, using the bounds $\left\||v| V_{N}\right\|_{3 / 2} \leq C N^{-1}$ and $\left\||v| V_{N}\right\|_{1} \leq C N^{-2}$. 
As for the second term on the r.h.s. of (6.14), we can bound it by applying a Schwarz inequality and Lemma 10.1 by

$$
\begin{aligned}
\mathrm{II} \leq & \int \mathrm{d} u \mathrm{~d} v \mathrm{~d} x_{2} \ldots \mathrm{d} x_{k} \mathrm{~d} \mathbf{x}_{k}^{\prime}, N\left|\left(W_{N}^{*} V_{N}\right)(v)\right|\left|J^{(k)}\left(\mathbf{x}_{k}^{\prime} ; u, x_{2}, \ldots, x_{k}\right)\right|\left|\xi_{\ell}^{(k+1)}\left(\mathbf{x}_{k}^{\prime}, u\right)\right|^{2} \\
& +\int \mathrm{d} u \mathrm{~d} v \mathrm{~d} x_{2} \ldots \mathrm{d} x_{k} \mathrm{~d} \mathbf{x}_{k}^{\prime} N\left|\left(W_{N}^{*} V_{N}\right)(v)\right|\left|J^{(k)}\left(\mathbf{x}_{k}^{\prime} ; u, x_{2}, \ldots, x_{k}\right)\right| \\
& \times\left|\left(W_{N,(1, k+1)}^{*} \xi_{\ell}^{(k+1)}\right)\left(u+v / 2, x_{2}, \ldots, x_{k}, u-v / 2\right)\right|^{2} \\
\leq & C_{k} N\left\|J^{(k)}\right\|\left\|W_{N}^{*} V_{N}\right\|_{1} \\
& \times\left\langle W_{N,(1, k+1)}^{*} \xi_{\ell}^{(k)},\left[\left(\nabla_{1} \cdot \nabla_{k+1}\right)^{2}-\Delta_{1}-\Delta_{k+1}+1\right] W_{N,(1, k+1)}^{*} \xi_{\ell}^{(k)}\right\rangle .
\end{aligned}
$$

Since, by Yajima's bounds (Proposition [5.1, part v)), $\left\|W_{N}^{*} V_{N}\right\|_{1} \leq C\left\|V_{N}\right\|_{1} \leq$ $C N^{-1}$, it follows that

$$
\mathrm{II} \leq C_{k}\left\|J^{(k)}\right\|\left\langle W_{N,(1, k+1)}^{*} \xi_{\ell}^{(k)},\left[\left(\nabla_{1} \cdot \nabla_{k+1}\right)^{2}-\Delta_{1}-\Delta_{k+1}+1\right] W_{N,(1, k+1)}^{*} \xi_{\ell}^{(k)}\right\rangle .
$$

Inserting this and (6.16) into the r.h.s. of (6.14), it follows from (6.13) after resumming over $\ell$ that

$$
\begin{aligned}
& \left|\operatorname{Tr} J^{(k)} N V_{N}\left(x_{1}-x_{k+1}\right) \gamma_{N, s}^{(k+1)}\right| \\
& \quad \leq C_{k}\left\|J^{(k)}\right\| \operatorname{Tr}\left(\left(\nabla_{1} \cdot \nabla_{k+1}\right)^{2}-\Delta_{1}-\Delta_{k+1}+1\right) W_{N,(1, k+1)}^{*} \gamma_{N, s}^{(k+1)} W_{N,(1, k+1)} \\
& \quad \leq C_{k}\left\|J^{(k)}\right\|
\end{aligned}
$$

for all $s \in \mathbb{R}$ (in the last line we used Proposition 5.3). Since the same bounds remain valid if we replace $x_{1}$ with an arbitrary $x_{j}, j=2, \ldots k$ (and also if the potential lies on the right of the marginal density $\gamma_{N, s}^{(k+1)}$ ), it follows that

$$
\sum_{j=1}^{k}\left|\operatorname{Tr} J^{(k)}\left[N V_{N}\left(x_{j}-x_{k+1}\right), \gamma_{N, s}^{(k+1)}\right]\right| \leq C_{k}\left\|J^{(k)}\right\|
$$

From (6.4), 6.5), 6.12), and (6.17), it follows that

$$
\left|\operatorname{Tr} J^{(k)}\left(\gamma_{N, t}^{(k)}-\gamma_{N, r}^{(k)}\right)\right| \leq C_{k}\left\|J^{(k)}\right\||t-s|
$$

This implies (6.2) and thus the equicontinuity of the sequence $\Gamma_{N, t}=\left\{\gamma_{N, t}^{(k)}\right\}_{k=1}^{N}$ with respect to the metric $\tau_{\text {prod }}$.

The proof of the fact that $\gamma_{\infty, t}^{(k)}$ is symmetric w.r.t. permutations, that it is nonnegative and such that $\operatorname{Tr} \gamma_{\infty, t}^{(k)} \leq 1$ can be found in [12, Theorem 6.1]. 


\section{Higher order a priori estimates on the Limit points $\Gamma_{\infty, t}$}

The goal of this section is to establish strong a priori bounds for the limit points $\Gamma_{\infty, t}$ of the sequence $\Gamma_{N, t}=\left\{\gamma_{N, t}^{(k)}\right\}_{k=1}^{N}$. As we did in [12], we will obtain strong a priori estimates on $\Gamma_{\infty, t}$ by proving higher order energy estimates, which compare the expectation of powers of the Hamiltonian $\left\langle\psi_{N}, H_{N}^{k} \psi_{N}\right\rangle$ with certain Sobolev norms of the $N$-particle wave function $\psi_{N}$. It turns out that the expectation of powers of the Hamiltonian can only control the Sobolev norms of $\psi_{N}$ in appropriate regions of the configuration space. To characterize these regions, we introduce the same cutoffs we used in 12 . For a given length scale $\ell>0$ (in our analysis, we will need that $\left.N^{-1 / 2} \ll \ell \ll N^{-1 / 3}\right)$, we set

$$
h(x):=e^{-\frac{\sqrt{x^{2}+\ell^{2}}}{\ell}} .
$$

Note that $h \simeq 0$ if $|x| \gg \ell$, and $h \simeq e^{-1}$ if $|x| \ll \ell$. For $i=1, \ldots, N$ we define the cutoff function

$$
\theta_{i}(\mathbf{x}):=\exp \left(-\frac{1}{\ell^{\varepsilon}} \sum_{j \neq i} h\left(x_{i}-x_{j}\right)\right)
$$

for some $\varepsilon>0$. Note that $\theta_{i}(\mathbf{x})$ is exponentially small if there is at least one other particle at distance of order $\ell$ from $x_{i}$, while $\theta_{i}(\mathbf{x})$ is exponentially close to 1 if there is no other particle near $x_{i}$ (on the length scale $\ell$ ). Next we define

$$
\theta_{i}^{(n)}(\mathbf{x}):=\theta_{i}(\mathbf{x})^{2^{n}}=\exp \left(-\frac{2^{n}}{\ell^{\varepsilon}} \sum_{j \neq i} h\left(x_{i}-x_{j}\right)\right)
$$

and their cumulative versions, for $n, k \in \mathbb{N}$,

$$
\Theta_{k}^{(n)}(\mathbf{x}):=\theta_{1}^{(n)}(\mathbf{x}) \ldots \theta_{k}^{(n)}(\mathbf{x})=\exp \left(-\frac{2^{n}}{\ell^{\varepsilon}} \sum_{i \leq k} \sum_{j \neq i} h\left(x_{i}-x_{j}\right)\right) .
$$

To cover all cases in one formula, we introduce the notation $\Theta_{k}^{(n)}=1$ for any $k \leq 0$, $n \in \mathbb{N}$. Some important properties of the function $\Theta_{k}^{(n)}$, used throughout the proof of Proposition 7.1, are collected, for completeness, in Lemma A.1.

Proposition 7.1. Suppose that $V \geq 0$, with $\left|\nabla^{\alpha} V(x)\right| \leq C$ for all $|\alpha| \leq 2$. Let $\psi \in L_{s}^{2}\left(\mathbb{R}^{3 N}\right)$ be a function symmetric in all its variables. Suppose that $\ell \gg N^{-1 / 2}$ (in the sense that there exists $\delta>0$ with $N^{1 / 2} \ell \geq N^{\delta}$ ). There exists $C_{0}>0$ such that for every integer $k \geq 1$ there exists $N_{0}=N_{0}(k)$ such that

$$
\left\langle\psi,\left(H_{N}+N\right)^{k} \psi\right\rangle \geq C_{0}^{k} N^{k} \int \mathrm{d} \mathbf{x} \Theta_{k-1}^{(k)}(\mathbf{x})\left|\nabla_{1} \ldots \nabla_{k} \psi(\mathbf{x})\right|^{2}
$$

for all $N \geq N_{0}$. 
Proof. We use induction over $k$. For $k=1$ the statement follows directly from $V_{N} \geq 0$, since on the symmetric subspace

$$
H_{N}+N \geq \sum_{i=1}^{N} \nabla_{i}^{*} \nabla_{i}=N \nabla_{j}^{*} \nabla_{j}
$$

for any fixed $j=1,2, \ldots, N$. We will present the $k=2$ case in detail and then comment on the general case. Set $T=H_{N}+N \geq 0$ for brevity and use the induction hypothesis

$$
\begin{aligned}
T^{2} \geq & C_{0} N T^{1 / 2} \nabla_{1}^{*} \nabla_{1} T^{1 / 2} \\
\geq & C_{0} N T^{1 / 2} \nabla_{1}^{*} \theta_{1}^{4} \nabla_{1} T^{1 / 2} \\
\geq & \frac{1}{2} C_{0} N \nabla_{1}^{*} T^{1 / 2} \theta_{1}^{4} T^{1 / 2} \nabla_{1}-C_{0} N\left[T^{1 / 2}, \nabla_{1}\right]^{*} \theta_{1}^{4}\left[T^{1 / 2}, \nabla_{1}\right] \\
\geq & \frac{1}{4} C_{0} N \nabla_{1}^{*} \theta_{1}^{2} T \theta_{1}^{2} \nabla_{1}-C_{0} N \nabla_{1}^{*}\left[T^{1 / 2}, \theta_{1}^{2}\right]^{*}\left[T^{1 / 2}, \theta_{1}^{2}\right] \nabla_{1} \\
& -C_{0} N\left[T^{1 / 2}, \nabla_{1}\right]^{*} \theta_{1}^{4}\left[T^{1 / 2}, \nabla_{1}\right] .
\end{aligned}
$$

In the first term we use the fact that $H_{N}+N \geq \sum_{j=2}^{N} \nabla_{j}^{*} \nabla_{j}$ to obtain

$$
\begin{aligned}
\frac{1}{4} C_{0} N \nabla_{1}^{*} \theta_{1}^{2} T \theta_{1}^{2} \nabla_{1} & \geq \frac{1}{4} C_{0} N(N-1) \nabla_{1}^{*} \theta_{1}^{2} \nabla_{2}^{*} \nabla_{2} \theta_{1}^{2} \nabla_{1} \\
& \geq \frac{1}{8} C_{0} N^{2} \nabla_{1}^{*} \nabla_{2}^{*} \theta_{1}^{4} \nabla_{2} \nabla_{1}-C_{0} N^{2} \nabla_{1}^{*}\left[\nabla_{2}, \theta_{1}^{2}\right]^{*}\left[\nabla_{2}, \theta_{1}^{2}\right] \nabla_{1}
\end{aligned}
$$

for all $N$ large enough. Since $\Theta_{1}^{(2)}=\theta_{1}^{4}$, we would obtain (7.5) for $k=2$ with $C_{0}<1 / 8$ once we show that the commutator terms in (7.7) and (7.8) are negligible.

The commutator in (17.8) on symmetric functions can be estimated by

$$
\begin{aligned}
C_{0} N^{2} \nabla_{1}^{*}\left[\nabla_{2}, \theta_{1}^{2}\right]^{*}\left[\nabla_{2}, \theta_{1}^{2}\right] \nabla_{1} & =\frac{C_{0} N^{2}}{N-1} \sum_{j=2}^{N} \nabla_{1}^{*}\left(\nabla_{j} \theta_{1}^{2}\right)^{2} \nabla_{1} \\
& \leq O\left(\ell^{-2} N^{-1}\right) N^{2} \nabla_{1}^{*} \nabla_{1} \leq O\left(\ell^{-2} N^{-1}\right) T^{2}=o(1) T^{2}
\end{aligned}
$$

where we used (A.1), recalling that $\theta_{1}^{2}=\Theta_{1}^{(1)}$, and where we also used $T \geq N$ and (7.6).

To estimate the two commutators in (7.7), we express

$$
\left[T^{1 / 2}, A\right]=\frac{1}{\pi} \int_{0}^{\infty} \frac{1}{T+s}[A, T] \frac{1}{T+s} s^{1 / 2} \mathrm{~d} s
$$

for any operator $A$.

To estimate the first commutator term in (7.7) we note that, by Schwarz inequality,

$$
\begin{aligned}
{\left[T^{1 / 2}, \theta_{1}^{2}\right]^{*}\left[T^{1 / 2}, \theta_{1}^{2}\right] \leq } & C(\log K) \int_{0}^{K} \frac{1}{T+s}\left[\theta_{1}^{2}, T\right]^{*} \frac{1}{(T+s)^{2}}\left[\theta_{1}^{2}, T\right] \frac{1}{T+s}\langle s\rangle^{2} \mathrm{~d} s \\
& +C \int_{K}^{\infty} \frac{1}{T+s}\left[\theta_{1}^{2}, T\right]^{*} \frac{1}{(T+s)^{2}}\left[\theta_{1}^{2}, T\right] \frac{1}{T+s}\langle s\rangle^{5 / 2} \mathrm{~d} s
\end{aligned}
$$


where $K=\exp \left(N^{\varepsilon}\right)$ for some $\varepsilon>0$. Estimating $(T+s)^{-2} \leq\langle s\rangle^{-2}($ using $T \geq N)$, we have

$$
\begin{aligned}
N \nabla_{1}^{*}\left[T^{1 / 2}, \theta_{1}^{2}\right]^{*}\left[T^{1 / 2}, \theta_{1}^{2}\right] \nabla_{1} \leq & c N^{1+\varepsilon} \int_{0}^{K} \nabla_{1}^{*} \frac{1}{T+s}\left[T, \theta_{1}^{2}\right]^{*}\left[T, \theta_{1}^{2}\right] \frac{1}{T+s} \nabla_{1} \mathrm{~d} s \\
& +c N \int_{K}^{\infty} \nabla_{1}^{*} \frac{1}{T+s}\left[T, \theta_{1}^{2}\right]^{*}\left[T, \theta_{1}^{2}\right] \frac{1}{T+s} \nabla_{1}\langle s\rangle^{1 / 2} \mathrm{~d} s,
\end{aligned}
$$

and we can estimate

$$
\begin{aligned}
& {\left[T, \theta_{1}^{2}\right]^{*}\left[T, \theta_{1}^{2}\right]=} \sum_{i, j}\left(2 \nabla_{j}^{*} \cdot\left(\nabla_{j} \theta_{1}^{2}\right)+\left(\Delta_{j} \theta_{1}^{2}\right)\right)\left(2\left(\nabla_{i} \theta_{1}^{2}\right) \cdot \nabla_{i}+\left(\Delta_{i} \theta_{1}^{2}\right)\right) \\
& \leq c \sum_{i, j}\left[\nabla_{j}^{*} \cdot\left(\nabla_{j} \theta_{1}^{2}\right)\left(\nabla_{i} \theta_{1}^{2}\right) \cdot \nabla_{i}\right. \\
&\left.\quad+\nabla_{i}^{*} \cdot\left|\Delta_{j} \theta_{1}^{2}\right| \nabla_{i}+\left|\nabla_{i} \theta_{1}^{2}\right|\left|\Delta_{j} \theta_{1}^{2}\right|\left|\nabla_{i} \theta_{1}^{2}\right|+\left|\Delta_{i} \theta_{1}^{2}\right|\left|\Delta_{j} \theta_{1}^{2}\right|\right] \\
& \leq c \sum_{i, j} \nabla_{i}^{*} \cdot\left(\left|\nabla_{j} \theta_{1}^{2}\right|^{2}+\left|\Delta_{j} \theta_{1}^{2}\right|\right) \nabla_{i}+c\left(\sum_{i}\left|\Delta_{i} \theta_{1}^{2}\right|\right)^{2} \\
& \leq c \ell^{-2} \sum_{i} \nabla_{i}^{*} \cdot \nabla_{i}+c \ell^{-4} \leq c \ell^{-2} T
\end{aligned}
$$

by using A.1 and A.2 and the fact that $\theta_{1}^{2}=\Theta_{1}^{(1)}$. Thus, the first commutator term in (7.7) is estimated as

$$
\begin{aligned}
N \nabla_{1}^{*}\left[T^{1 / 2}, \theta_{1}^{2}\right]^{*}\left[T^{1 / 2}, \theta_{1}^{2}\right] \nabla_{1} \leq & O\left(N^{1+\varepsilon} \ell^{-2}\right) \int_{0}^{K} \nabla_{1}^{*} \frac{1}{T+s} T \frac{1}{T+s} \nabla_{1} \mathrm{~d} s \\
& +O\left(N \ell^{-2}\right) \int_{K}^{\infty} \nabla_{1}^{*} \frac{1}{T+s} T \frac{1}{T+s} \nabla_{1}\langle s\rangle^{1 / 2} \mathrm{~d} s \\
\leq & O\left(N^{1+\varepsilon} \ell^{-2}\right) \nabla_{1}^{*} \nabla_{1}+O\left(N \ell^{-2} K^{-1 / 2}\right) \nabla_{1}^{*} T \nabla_{1} \\
\leq & O\left(N^{\varepsilon} \ell^{-2}\right) T+o(1) T^{2} \\
\leq & o(1) T^{2}
\end{aligned}
$$

if we choose $\varepsilon>0$ so small that $\ell^{-2} \ll N^{1-\varepsilon}$. When estimating the term $\nabla_{1}^{*} T \nabla_{1}$ in the last step, we could afford estimating any commutators, since $K^{-1 / 2}$ is exponentially small:

$$
\begin{aligned}
\nabla_{1}^{*} T \nabla_{1} & =\frac{1}{N} \sum_{j} \nabla_{j}^{*} T \nabla_{j}=\frac{1}{N} \sum_{j}\left[\left(-\Delta_{j}\right) T-\nabla_{j}^{*} \cdot \sum_{i}\left(\nabla V_{N}\right)\left(x_{j}-x_{i}\right)\right] \\
& =N^{-1} T^{2}-N^{-1} \sum_{i j}\left[V_{N}\left(x_{i}-x_{j}\right) T-\nabla_{j}^{*} \cdot\left(\nabla V_{N}\right)\left(x_{j}-x_{i}\right)\right] \\
& \leq 2 N^{-1} T^{2}+O\left(N^{8}\right)
\end{aligned}
$$

using the fact that $\left|V_{N}(x)\right| \leq C N^{2}$ and $\left|\nabla V_{N}(x)\right| \leq C N^{3}$ for all $x \in \mathbb{R}^{3}$. 
Finally, we estimate the second commutator term in (7.7) by again using the Schwarz inequality in (7.10), but this time we do not split the integration:

$$
\begin{aligned}
& {\left[T^{1 / 2}, \nabla_{1}\right]^{*} \theta_{1}^{4}\left[T^{1 / 2}, \nabla_{1}\right]} \\
& \leq c \int_{0}^{\infty} \frac{1}{T+s}\left[\nabla_{1}, T\right]^{*} \frac{1}{T+s} \theta_{1}^{4} \frac{1}{T+s}\left[\nabla_{1}, T\right] \frac{1}{T+s}\langle s\rangle^{5 / 2} \mathrm{~d} s \\
& \leq c N \sum_{i \neq 1} \int_{0}^{\infty} \frac{1}{T+s}\left(\nabla V_{N}\right)\left(x_{1}-x_{i}\right) \frac{1}{T+s} \theta_{1}^{4} \frac{1}{T+s} \\
& \times\left(\nabla V_{N}\right)\left(x_{1}-x_{i}\right) \frac{1}{T+s}\langle s\rangle^{5 / 2} \mathrm{~d} s
\end{aligned}
$$

where we used $\left[\nabla_{1}, T\right]=\sum_{i \neq 1}\left(\nabla V_{N}\right)\left(x_{1}-x_{i}\right)$.

Since $T_{0}=\sum_{j}-\Delta_{j}+N$ is a positivity preserving operator and $V \geq 0, T$ is also positivity preserving and its resolvent kernel satisfies

$$
\frac{1}{T+s}(\mathbf{x} ; \mathbf{y}) \leq \frac{1}{T_{0}+s}(\mathbf{x} ; \mathbf{y})
$$

and thus

$$
\begin{aligned}
I & :=\left\|\left(\nabla V_{N}\right)\left(x_{1}-x_{i}\right) \frac{1}{T+s} \theta_{1}^{4} \frac{1}{T+s}\left(\nabla V_{N}\right)\left(x_{1}-x_{i}\right)\right\| \\
& \leq\left\|\left|\left(\nabla V_{N}\right)\left(x_{1}-x_{i}\right)\right| \frac{1}{T_{0}+s} e^{-4 \ell^{-\varepsilon} h\left(x_{1}-x_{i}\right)} \frac{1}{T_{0}+s}\left|\left(\nabla V_{N}\right)\left(x_{1}-x_{i}\right)\right|\right\|,
\end{aligned}
$$

where we also estimated $\theta_{1}$ by keeping only one summand in its definition (7.2). Introducing the variable $y=x_{1}-x_{i}$ and observing that

$$
L^{2}\left(\mathbb{R}^{3 N}, \mathrm{~d} \mathbf{x}\right) \simeq L^{2}\left(\mathbb{R}^{3}, \mathrm{~d} y ; L^{2}\left(\mathbb{R}^{3(N-1)}, \mathrm{d} z \mathrm{~d} x_{2} \ldots \widehat{\mathrm{d} x_{i}} \ldots \mathrm{d} x_{N}\right)\right)
$$

(where the hat means that the variable $x_{i}$ is omitted), we obtain that

$$
\begin{aligned}
I & \leq \sup _{M \geq 0}\left\|\left|\left(\nabla V_{N}\right)(y)\right| \frac{1}{-\Delta_{y}+M+N+s} e^{-4 \ell^{-\varepsilon} h(y)} \frac{1}{\Delta_{y}+M+N+s}\left|\left(\nabla V_{N}\right)(y)\right|\right\| \\
& \leq \sup _{M \geq 0}\left\|\left|\left(\nabla V_{N}\right)(y)\right| \frac{1}{-\Delta_{y}+M+N+s} e^{-4 \ell^{-\varepsilon} h(y)} \frac{1}{\Delta_{y}+M+N+s}\left|\left(\nabla V_{N}\right)(y)\right|\right\|_{\mathrm{HS}}
\end{aligned}
$$

where the norms on the last two lines are, respectively, the operator norm and the Hilbert-Schmidt norm of an operator over $L^{2}\left(\mathbb{R}^{3}, \mathrm{~d} y\right)$. The last equation implies that

$$
\begin{aligned}
I & \leq \int \mathrm{d} y \mathrm{~d} y^{\prime} e^{-4 \ell^{-\varepsilon} h(y)}\left|\frac{1}{\Delta+M+N+s}\left(y, y^{\prime}\right)\right|^{2}\left|\nabla V_{N}\left(y^{\prime}\right)\right|^{2} \\
& \leq \int \mathrm{d} y \mathrm{~d} y^{\prime} e^{-4 \ell^{-\varepsilon} h(y)} \frac{e^{-2 \sqrt{N+s}\left|y-y^{\prime}\right|}}{\left|y-y^{\prime}\right|^{2}}\left|\nabla V_{N}\left(y^{\prime}\right)\right|^{2} \\
& \leq O\left(e^{-\ell^{-\varepsilon}}\right),
\end{aligned}
$$

since $h \approx e^{-1}$ on the support of $V_{N}$. We will use this bound for $s \leq K:=\exp \left(c \ell^{-\varepsilon}\right)$ with a sufficiently small $c>0$. From (7.16), we also have the trivial bound

$$
I \leq \frac{N^{6}}{\langle s\rangle^{2}}
$$


that will be used for large $s$. Inserting these estimates into (7.15), we have

$$
\begin{aligned}
{\left[T^{1 / 2}, \nabla_{1}\right]^{*} \theta_{1}^{4}\left[T^{1 / 2}, \nabla_{1}\right] } & \leq O\left(N^{2} e^{-\ell^{-\varepsilon}}\right) \int_{0}^{K} \frac{\langle s\rangle^{5 / 2} \mathrm{~d} s}{(T+s)^{2}}+O\left(N^{8}\right) \int_{K}^{\infty} \frac{\langle s\rangle^{1 / 2} \mathrm{~d} s}{(T+s)^{2}} \\
& =O\left(e^{-c \ell^{-\varepsilon}}\right),
\end{aligned}
$$

i.e. this commutator term is subexponentially small in $N$, and this completes the proof of (7.5) for $k=2$.

The proof for general $k>2$ follows the same pattern as for $k=2$. Introduce the notation

$$
D_{k}=\nabla_{1} \nabla_{2} \ldots \nabla_{k}
$$

We recall the summation convention: for any operator $A$, we denote

$$
D_{k}^{*} A D_{k}:=\sum_{\alpha_{1}=1}^{3} \ldots \sum_{\alpha_{k}=1}^{3} \nabla_{x_{1, \alpha_{1}}}^{*} \ldots \nabla_{x_{k, \alpha_{k}}}^{*} A \nabla_{x_{k, \alpha_{k}}} \ldots \nabla_{x_{1, \alpha_{1}}}
$$

where $x_{j}=\left(x_{j, 1}, x_{j, 2}, x_{j, 3}\right)$ are the three coordinates of $x_{j} \in \mathbb{R}^{3}$.

Using the induction hypothesis, $\Theta_{k-1}^{(k)} \geq\left[\Theta_{k}^{(k)}\right]^{2}=\Theta_{k}^{(k+1)}$ and (7.6) we obtain, similarly to (7.7) and (7.8),

$$
\begin{aligned}
T^{k+1} \geq & C_{0}^{k} N^{k} T^{1 / 2} D_{k}^{*} \Theta_{k-1}^{(k)} D_{k} T^{1 / 2} \\
\geq & C_{0}^{k} N^{k} T^{1 / 2} D_{k}^{*}\left[\Theta_{k}^{(k)}\right]^{2} D_{k} T^{1 / 2} \\
\geq & \frac{1}{8} C_{0}^{k} N^{k+1} D_{k+1}^{*} \Theta_{k}^{(k+1)} D_{k+1}-C_{0}^{k} N^{k} D_{k}^{*}\left[T^{1 / 2}, \Theta_{k}^{(k)}\right]^{*}\left[T^{1 / 2}, \Theta_{k}^{(k)}\right] D_{k} \\
& -C_{0}^{k} N^{k}\left[T^{1 / 2}, D_{k}\right]^{*} \Theta_{k}^{(k+1)}\left[T^{1 / 2}, D_{k}\right] \\
& -C_{0}^{k} N^{k+1} D_{k}^{*}\left[\nabla_{k+1}, \Theta_{k}^{(k)}\right]^{*}\left[\nabla_{k+1}, \Theta_{k}^{(k)}\right] D_{k},
\end{aligned}
$$

for all $N$ sufficiently large (depending on $k$ ). The first term gives the desired result if $C_{0}<1 / 8$; in the sequel we show that all three commutator terms are negligible.

The first commutator in (7.19) is estimated exactly as the first commutator in (7.7), after replacing $\theta_{1}^{2}=\Theta_{1}^{(1)}$ with $\Theta_{k}^{(k)}$. The estimates (7.11) are (7.12) are identical for $k>1$ as well. In the key estimate (7.13), the only properties we used regarding $\theta_{1}^{2}=\Theta_{1}^{(1)}$ from Lemma A.1 were those that hold for $\Theta_{k}^{(k)}$ as well.

The last commutator in (7.19) can be estimated similarly to (7.9) by using (A.1):

$$
\begin{aligned}
C_{0}^{k} N^{k+1} D_{k}^{*}\left[\nabla_{k+1}, \Theta_{k}^{(k)}\right]^{*}\left[\nabla_{k+1}, \Theta_{k}^{(k)}\right] D_{k} & =\frac{C_{0}^{k} N^{k+1}}{N-k} \sum_{j=k+1}^{N} D_{k}^{*}\left(\nabla_{j} \Theta_{k}^{(k)}\right)^{2} D_{k} \\
& \leq O\left(\ell^{-2} N^{-1}\right) N^{k+1} D_{k}^{*} \Theta_{k}^{(k-1)} D_{k} \\
& \leq O_{k}\left(\ell^{-2} N^{-1}\right) N T^{k}=o_{k}(1) T^{k+1}
\end{aligned}
$$

by the induction hypothesis and $T \geq N$ (here we use the notation $f=o_{k}(g)$ if $f / g \rightarrow 0$ as $N \rightarrow \infty$ for fixed $k$; analogously for $\left.f=O_{k}(g)\right)$.

Finally, the estimate of the second commutator in (7.19) is similar to that of the second commutator in (7.7), but more commutators need to be computed. Similarly 
to (7.15) and taking the permutation symmetry into account, we have

$$
\begin{aligned}
& {\left[T^{1 / 2}, D_{k}\right]^{*} \Theta_{k}^{(k+1)}\left[T^{1 / 2}, D_{k}\right]} \\
& \leq C_{k} N \sum_{i \neq k} \int_{0}^{\infty} \frac{1}{T+s} D_{k-1}^{*}\left(\nabla V_{N}\right)\left(x_{k}-x_{i}\right) \frac{1}{T+s} \Theta_{k}^{(k+1)} \\
& \quad \times \frac{1}{T+s}\left(\nabla V_{N}\right)\left(x_{k}-x_{i}\right) D_{k-1} \frac{1}{T+s}\langle s\rangle^{5 / 2} \mathrm{~d} s \\
& \quad+C_{k} \int_{0}^{\infty} \frac{1}{T+s} D_{k-2}^{*}\left(\nabla^{2} V_{N}\right)\left(x_{k}-x_{k-1}\right) \frac{1}{T+s} \Theta_{k}^{(k+1)} \\
& \quad \times \frac{1}{T+s}\left(\nabla^{2} V_{N}\right)\left(x_{k}-x_{k-1}\right) D_{k-2} \frac{1}{T+s}\langle s\rangle^{5 / 2} \mathrm{~d} s
\end{aligned}
$$

We will need the following lemma whose proof is postponed.

Lemma 7.2. Let $\psi \in L_{s}^{2}\left(\mathbb{R}^{3 N}\right)$ be a function symmetric in all its variables and let $\delta>0$. Choose a strictly increasing sequence of positive constants $\left\{c_{k}\right\}_{k \geq 1}$. Then for every integer $k \geq 1$ there exists $N_{0}=N_{0}(k, \delta)$ such that

$$
\left\langle\psi,\left(H_{N}+N\right)^{k} \psi\right\rangle \geq e^{-c_{k} N^{\delta}} \int \mathrm{d} \mathbf{x}\left|\nabla_{1} \ldots \nabla_{k} \psi(\mathbf{x})\right|^{2}
$$

for all $N \geq N_{0}$.

We demonstrate the estimate of the first term in (7.21); the second one is similar. Using $\Theta_{k}^{(k+1)} \leq e^{-\ell^{-\varepsilon} h\left(x_{k}-x_{i}\right)}$, we obtain, similarly to (7.16)-(7.18) that

$$
I:=\left\|\left(\nabla V_{N}\right)\left(x_{k}-x_{i}\right) \frac{1}{T+s} \Theta_{k}^{(k+1)} \frac{1}{T+s}\left(\nabla V_{N}\right)\left(x_{k}-x_{i}\right)\right\| \leq O\left(e^{-\ell^{-\varepsilon}}\right)
$$

and also

$$
I \leq \frac{N^{6}}{\langle s\rangle^{2}}
$$

Let $K:=\exp \left(c \ell^{-\varepsilon}\right)$ with a sufficiently small $c>0$. Choosing a sufficiently small $\delta$, so that $N^{\delta} \ll \ell^{-\varepsilon}$, by using (7.22) we have

$$
\begin{aligned}
& \int_{0}^{\infty} \frac{1}{T+s} D_{k-1}^{*}\left(\nabla V_{N}\right)\left(x_{k}-x_{i}\right) \frac{1}{T+s} \Theta_{k}^{(k+1)} \\
& \quad \times \frac{1}{T+s}\left(\nabla V_{N}\right)\left(x_{k}-x_{i}\right) D_{k-1} \frac{1}{T+s}\langle s\rangle^{5 / 2} \mathrm{~d} s \\
& \leq O\left(e^{-\ell^{-\varepsilon}+c_{k-1} N^{\delta}}\right) \int_{0}^{K} \frac{T^{k-1}}{(T+s)^{2}}\langle s\rangle^{5 / 2} \mathrm{~d} s \\
&+O\left(e^{c_{k-1} N^{\delta}}\right) \int_{K}^{\infty} \frac{T^{k-1}}{(T+s)^{2}} \frac{N^{6}}{\langle s\rangle^{2}}\langle s\rangle^{5 / 2} \mathrm{~d} s \\
& \leq O\left(e^{-c^{\prime} \ell^{-\varepsilon}}\right) T^{k-1} \leq o(1) T^{k+1} .
\end{aligned}
$$

This completes the proof of Proposition 7.1

Proof of Lemma 7.2. We proceed by a step-two induction on $k$; for $k=1$ the claim follows from (7.6). We now consider the $k=2$ case. Similarly to (5.4), but also 
keeping the $h_{j}^{2}$ terms in the expansion of $H_{N}^{2}$, we find

$$
\begin{aligned}
T^{2} \geq & N(N-1)\left(-\Delta_{1}+\frac{1}{2} V_{N}\left(x_{1}-x_{2}\right)\right)\left(-\Delta_{2}+\frac{1}{2} V_{N}\left(x_{1}-x_{2}\right)\right) \\
& +N\left(-\Delta_{1}+\frac{1}{2} V_{N}\left(x_{1}-x_{2}\right)\right)^{2} \\
\geq & \left(N^{2} / 2\right)\left(D_{2}^{*} D_{2}-2 \nabla_{1}^{*} \nabla_{1}-2 \nabla_{2}^{*} \nabla_{2}-4\left\|\nabla V_{N}\right\|_{\infty}^{2}\right) \\
& +N\left(\left(\nabla_{1}^{*} \nabla_{1}\right)^{2}-2 \nabla_{1}^{*} \nabla_{1}-4\left\|\nabla V_{N}\right\|_{\infty}^{2}\right) \\
\geq & \left(N^{2} / 2\right) D_{2}^{*} D_{2}+N\left(\nabla_{1}^{*} \nabla_{1}\right)^{2}-C N^{2} \nabla_{1}^{*} \nabla_{1}-C N^{8} \\
\geq & \left(N^{2} / 2\right) D_{2}^{*} D_{2}-O\left(N^{8}\right) .
\end{aligned}
$$

Combining this bound with $T^{2} \geq N^{2}$, it follows that

$$
T^{2} \geq c N^{-4} D_{2}^{*} D_{2}
$$

for a sufficiently small positive $c$.

Now we show how to go from $k$ to $k+2$. By the induction hypothesis, we have

$$
T^{k+2} \geq e^{-c_{k} N^{\delta}} T D_{k}^{*} D_{k} T \geq e^{-c_{k} N^{\delta}}\left(\frac{1}{2} D_{k}^{*} T^{2} D_{k}-2\left[D_{k}, T\right]^{*}\left[D_{k}, T\right]\right) .
$$

In the first term we can use (7.25) in the form $T^{2} \geq c N^{-4} \nabla_{k+1}^{*} \nabla_{k+2}^{*} \nabla_{k+2} \nabla_{k+1}$, which holds for all $N$ large enough. (Because of the factors $D_{k}$, we only have symmetry on the last $N-k$ variables. This means that instead of (7.24), we are going to obtain $T^{2} \geq(N-k)(N-k-1) \Delta_{k+1} \Delta_{k+2} \geq\left(N^{2} / 2\right) \Delta_{k+1} \Delta_{k+2}$ for all $N$ large enough.)

The commutator term, after several Schwarz inequalities, can be estimated as

$$
\begin{aligned}
{\left[D_{k}, T\right]^{*}\left[D_{k}, T\right] } & \leq C_{k}\left(N^{2} D_{k-1}^{*}\left\|\nabla V_{N}\right\|^{2} D_{k-1}+D_{k-2}^{*}\left\|\nabla^{2} V_{N}\right\|^{2} D_{k-2}\right) \\
& \leq C_{k} N^{8}\left(D_{k-1}^{*} D_{k-1}+D_{k-2}^{*} D_{k-2}\right) \\
& \leq C_{k} N^{8} e^{c_{k-1} N^{\delta}} T^{k-1} \leq C_{k} N^{8} e^{c_{k-1} N^{\delta}} T^{k+2}
\end{aligned}
$$

where we used the induction hypothesis for $k-1$ and $k-2$ and, by convention, $D_{m}=1$ for $m \leq 0$. Inserting this estimate into (7.26), we obtain

$$
T^{k+2} \geq c N^{-4} e^{-c_{k} N^{\delta}} D_{k+2}^{*} D_{k+2}-C_{k} N^{8} e^{-\left(c_{k}-c_{k-1}\right) N^{\delta}} T^{k+2} .
$$

Since $c_{k}$ is strictly increasing, we obtain (7.22) for $k+2$.

Actually the proof shows that a sufficiently large $k$-dependent negative power, $N^{-\beta_{k}}$, would suffice on the r.h.s. of (7.22) instead of the subexponentially small prefactor.

The higher order energy estimates proved in Proposition 7.1 are used to show the following strong a priori estimates on the limit points $\Gamma_{\infty, t}=\left\{\gamma_{\infty, t}^{(k)}\right\}_{k \geq 1}$ of the sequence $\Gamma_{N, t}$. 
Theorem 7.3. Suppose that the assumptions of Theorem 3.2 are satisfied and fix $T>0$. Assume moreover that $\Gamma_{\infty, t}^{(k)}=\left\{\gamma_{\infty, t}^{(k)}\right\}_{k \geq 1} \in \bigoplus_{k \geq 1} C\left([0, T], \mathcal{L}_{k}^{1}\right)$ is a limit point of the sequence $\Gamma_{N, t}=\left\{\gamma_{N, t}^{(k)}\right\}_{k=1}^{N}$ with respect to the topology $\tau_{\text {prod }}$. Then

$$
\operatorname{Tr}\left(1-\Delta_{1}\right) \ldots\left(1-\Delta_{k}\right) \gamma_{\infty, t}^{(k)} \leq C^{k}
$$

for all $k \geq 1$ and $t \in[0, T]$.

Proof. Theorem 7.3 follows from the higher order energy estimates of Theorem 7.1 The proof of this fact can be found in [12, Proposition 6.3].

\section{Convergence to the infinite hierarchy}

In order to prove Theorem 3.2, we need to prove the convergence of the BBGKY hierarchy towards a hierarchy of infinitely many equations. In the argument, we will make use of the a priori bounds from Proposition 5.3 and Theorem 7.3 for $k=2$.

Theorem 8.1. Suppose that the assumptions of Theorem 3.2 are satisfied and fix $T>0$. Suppose that $\Gamma_{\infty, t}=\left\{\gamma_{\infty, t}^{(k)}\right\}_{k \geq 1} \in \bigoplus_{k \geq 1} C\left([0, T], \mathcal{L}_{k}^{1}\right)$ is a limit point of $\Gamma_{N, t}=\left\{\gamma_{N, t}^{(k)}\right\}_{k=1}^{N}$ with respect to the topology $\tau_{\text {prod. }}$. Then $\Gamma_{\infty, t}$ is a solution to the infinite hierarchy

(8.1) $\gamma_{\infty, t}^{(k)}=\mathcal{U}^{(k)}(t) \gamma_{\infty, 0}^{(k)}-8 \pi a_{0} i \sum_{j=1}^{k} \int_{0}^{t} \mathrm{~d} s \mathcal{U}^{(k)}(t-s) \operatorname{Tr}_{k+1}\left[\delta\left(x_{j}-x_{k+1}\right), \gamma_{\infty, s}^{(k+1)}\right]$

with initial data $\gamma_{\infty, 0}^{(k)}=|\varphi\rangle\left\langle\left.\varphi\right|^{\otimes k}\right.$. Here $\mathcal{U}^{(k)}(t)$ denotes the free evolution of $k$ particles defined in (4.5).

Proof. Fix $k \geq 1$. Passing to an appropriate subsequence, we can assume that, for every $J^{(k)} \in \mathcal{K}_{k}$,

$$
\sup _{t \in[0, T]} \operatorname{Tr} J^{(k)}\left(\gamma_{N, t}^{(k)}-\gamma_{\infty, t}^{(k)}\right) \rightarrow 0 \quad \text { as } N \rightarrow \infty .
$$

We will prove (8.1) by testing the limit point against a certain class of observables that is dense in $\mathcal{K}_{k}$. To characterize the class of observables we are going to consider, we define, for an arbitrary integer $k \geq 1$,

$$
\Omega_{k}:=\prod_{j=1}^{k}\left(\left\langle x_{j}\right\rangle+\left\langle i \nabla_{j}\right\rangle\right) .
$$

We will consider $J^{(k)} \in \mathcal{K}_{k}$ such that

$$
\left\|\Omega_{k}^{7} J^{(k)} \Omega_{k}^{7}\right\|_{\mathrm{HS}}<\infty
$$

where $\|A\|_{\mathrm{HS}}$ denotes the Hilbert-Schmidt norm of the operator $A$. Note that the set of observables $J^{(k)}$ satisfying the condition (8.3) is a dense subset of $\mathcal{K}_{k}$. Moreover, using the fact that $e^{i \Delta_{j} t}\left\langle x_{j}\right\rangle e^{-i \Delta_{j} t}=\left\langle x_{j}-i t \nabla_{j}\right\rangle$, it follows that

$$
\left\|\Omega_{k}^{7} \mathcal{U}^{(k)}(t) J^{(k)} \Omega_{k}^{7}\right\|_{\mathrm{HS}} \leq C(1+|t|)^{7}\left\|\Omega_{k}^{7} J^{(k)} \Omega_{k}^{7}\right\|_{\mathrm{HS}} .
$$

Note also that, with the norm $\left\|J^{(k)}\right\|$ defined in (6.3), we have

$$
\left\|J^{(k)}\right\| \leq C_{k}\left\|\Omega_{k}^{7} J^{(k)} \Omega_{k}^{7}\right\|_{\mathrm{HS}}
$$


for a constant $C_{k}$ only depending on $k$ (see [12], Eq. (7.8)). Combining (8.4) with (8.5), we also have

$$
\left\|\mathcal{U}^{(k)}(t) J^{(k)}\right\| \leq C_{k}(1+|t|)^{7}\left\|\Omega_{k}^{7} J^{(k)} \Omega_{k}^{7}\right\|_{\mathrm{HS}} .
$$

In order to prove Theorem 8.1 it is enough to show that, for every $J^{(k)} \in \mathcal{K}_{k}$ satisfying (8.3),

$$
\operatorname{Tr} J^{(k)} \gamma_{\infty, 0}^{(k)}=\operatorname{Tr} J^{(k)}|\varphi\rangle\left\langle\left.\varphi\right|^{\otimes k}\right.
$$

and

$$
\begin{aligned}
\operatorname{Tr} J^{(k)} \gamma_{\infty, t}^{(k)}= & \operatorname{Tr} J^{(k)} \mathcal{U}^{(k)}(t) \gamma_{\infty, 0}^{(k)} \\
& -8 \pi a_{0} i \sum_{j=1}^{k} \int_{0}^{t} \mathrm{~d} s \operatorname{Tr} J^{(k)} \mathcal{U}^{(k)}(t-s)\left[\delta\left(x_{j}-x_{k+1}\right), \gamma_{\infty, s}^{(k+1)}\right]
\end{aligned}
$$

for all $t \in[0, T]$.

The relation (8.7) follows from the assumption (3.5) and from (8.2).

In order to prove (8.8), we fix $t \in[0, T]$, we rewrite the BBGKY hierarchy (2.1) in integral form and we test it against the observable $J^{(k)}$. We obtain

$$
\begin{aligned}
\operatorname{Tr} J^{(k)} \gamma_{N, t}^{(k)}= & \operatorname{Tr} J^{(k)} \mathcal{U}^{(k)}(t) \gamma_{N, 0}^{(k)}-i \sum_{i<j}^{k} \int_{0}^{t} \mathrm{~d} s \operatorname{Tr} J^{(k)} \mathcal{U}^{(k)}(t-s)\left[V_{N}\left(x_{i}-x_{j}\right), \gamma_{N, s}^{(k)}\right] \\
& -i(N-k) \sum_{j=1}^{k} \int_{0}^{t} \mathrm{~d} s \operatorname{Tr} J^{(k)} \mathcal{U}^{(k)}(t-s)\left[V_{N}\left(x_{j}-x_{k+1}\right), \gamma_{N, s}^{(k+1)}\right]
\end{aligned}
$$

From (8.2) it follows immediately that

$$
\operatorname{Tr} J^{(k)} \gamma_{N, t}^{(k)} \rightarrow \operatorname{Tr} J^{(k)} \gamma_{\infty, t}^{(k)}
$$

and also that, as $N \rightarrow \infty$,

$$
\begin{aligned}
\operatorname{Tr} J^{(k)} \mathcal{U}^{(k)}(t) \gamma_{N, 0}^{(k)}= & \operatorname{Tr}\left(\mathcal{U}^{(k)}(-t) J^{(k)}\right) \gamma_{N, 0}^{(k)} \\
& \rightarrow \operatorname{Tr}\left(\mathcal{U}^{(k)}(-t) J^{(k)}\right) \gamma_{\infty, 0}^{(k)}=\operatorname{Tr} J^{(k)} \mathcal{U}^{(k)}(t) \gamma_{\infty, 0}^{(k)} .
\end{aligned}
$$

Here we used the fact that, if $J^{(k)} \in \mathcal{K}_{k}$, then also $\mathcal{U}^{(k)}(-t) J^{(k)} \in \mathcal{K}_{k}$.

Next we consider the second term on the r.h.s. of (8.9) and we prove that it converges to zero, as $N \rightarrow \infty$. To this end, we note that, setting $J_{t}^{(k)}=\mathcal{U}^{(k)}(t) J^{(k)}$, we have

$$
\sum_{i<j}^{k} \operatorname{Tr} J^{(k)} \mathcal{U}^{(k)}(t-s)\left[V_{N}\left(x_{i}-x_{j}\right), \gamma_{N, s}^{(k)}\right]=\sum_{i<j}^{k} \operatorname{Tr} J_{s-t}^{(k)}\left[V_{N}\left(x_{i}-x_{j}\right), \gamma_{N, s}^{(k)}\right],
$$

and therefore, from (6.12) and (8.6), we obtain that

$$
\sum_{i<j}^{k}\left|\operatorname{Tr} J^{(k)} \mathcal{U}^{(k)}(t-s)\left[V_{N}\left(x_{i}-x_{j}\right), \gamma_{N, s}^{(k)}\right]\right| \leq \frac{C_{k}\left\|J_{s-t}^{(k)}\right\|}{N} \leq \frac{C_{k}\left(1+T^{7}\right)\left\|\Omega_{k}^{7} J^{(k)} \Omega_{k}^{7}\right\|_{\mathrm{HS}}}{N}
$$


for all $0 \leq s \leq t \leq T$. This implies that for all $k \in \mathbb{N}$, for all $t \in[0, T]$ and for all $J^{(k)}$ such that $(8.3)$ is true,

$$
\sum_{i<j}^{k} \int_{0}^{t} \mathrm{~d} s \operatorname{Tr} J^{(k)} \mathcal{U}^{(k)}(t-s)\left[V_{N}\left(x_{i}-x_{j}\right), \gamma_{N, s}^{(k)}\right] \rightarrow 0
$$

as $N \rightarrow \infty$.

Finally we consider the last term on the r.h.s. of (8.9). First of all, we observe that, for every $k \in \mathbb{N}, t \in[0, T]$ and $J^{(k)} \in \mathcal{K}_{k}$ such that (8.3) is satisfied, we have

$$
k \sum_{j=1}^{k} \int_{0}^{t} \mathrm{~d} s \operatorname{Tr} J^{(k)} \mathcal{U}^{(k)}(t-s)\left[V_{N}\left(x_{j}-x_{k+1}\right), \gamma_{N, s}^{(k+1)}\right] \rightarrow 0
$$

as $N \rightarrow \infty$. This follows (similarly to (8.12) ) from (6.17), and from the bound (8.6).

It remains to show that, for every fixed $k \in \mathbb{N}, t \in[0, T]$, and for every $J^{(k)} \in \mathcal{K}_{k}$ with (8.3)

$$
\begin{aligned}
N \sum_{j=1}^{k} \int_{0}^{t} \mathrm{~d} s & \operatorname{Tr} J^{(k)} \mathcal{U}^{(k)}(t-s)\left[V_{N}\left(x_{j}-x_{k+1}\right), \gamma_{N, s}^{(k+1)}\right] \\
& \rightarrow 8 \pi a_{0} \sum_{j=1}^{k} \int_{0}^{t} \mathrm{~d} s \operatorname{Tr} J^{(k)} \mathcal{U}^{(k)}(t-s)\left[\delta\left(x_{j}-x_{k+1}\right), \gamma_{\infty, s}^{(k+1)}\right]
\end{aligned}
$$

as $N \rightarrow \infty$. To prove (8.14), we fix $s \in[0, t]$, and we consider, for example, the contribution with $j=1$. We write

$$
\begin{aligned}
\operatorname{Tr} J^{(k)} \mathcal{U}^{(k)}(t-s) N & V_{N}\left(x_{1}-x_{k+1}\right) \gamma_{N, s}^{(k+1)} \\
& =\operatorname{Tr} J_{s-t}^{(k)} N V_{N}\left(x_{1}-x_{k+1}\right) W_{N,(1, k+1)} W_{N,(1, k+1)}^{*} \gamma_{N, s}^{(k+1)}
\end{aligned}
$$

where $W_{N,(1, k+1)}$ denotes the wave operator associated with the Hamiltonian $-\Delta+$ $(1 / 2) V_{N}$ acting only on the variable $x_{k+1}-x_{1}$ (as defined in (5.2)). Therefore, if we choose a probability density $h \in L^{1}\left(\mathbb{R}^{3}\right)$, with $h \geq 0, \int \mathrm{d} x h(x)=1$, and we denote $h_{\alpha}(x)=\alpha^{-3} h(x / \alpha)$ for all $\alpha>0$, we have

$$
\begin{aligned}
& \mid \operatorname{Tr} J^{(k)} \mathcal{U}^{(k)}(t-s) N V_{N}\left(x_{1}-x_{k+1}\right) \gamma_{N, s}^{(k+1)} \\
& \quad-8 \pi a_{0} \operatorname{Tr} J^{(k)} \mathcal{U}^{(k)}(t-s) \delta\left(x_{1}-x_{k+1}\right) \gamma_{\infty, s}^{(k+1)} \mid \\
& \leq\left|\operatorname{Tr} J_{s-t}^{(k)}\left[N V_{N}\left(x_{1}-x_{k+1}\right) W_{N,(1, k+1)}-8 \pi a_{0} \delta\left(x_{1}-x_{k+1}\right)\right] W_{N,(1, k+1)}^{*} \gamma_{N, s}^{(k+1)}\right| \\
& \quad+8 \pi a_{0}\left|\operatorname{Tr} J_{s-t}^{(k)}\left[\delta\left(x_{1}-x_{k+1}\right)-h_{\alpha}\left(x_{1}-x_{k+1}\right)\right] W_{N,(1, k+1)}^{*} \gamma_{N, s}^{(k+1)}\right| \\
& \quad+8 \pi a_{0}\left|\operatorname{Tr} J_{s-t}^{(k)} h_{\alpha}\left(x_{1}-x_{k+1}\right)\left(W_{N,(1, k+1)}^{*}-1\right) \gamma_{N, s}^{(k+1)}\right| \\
& \quad+8 \pi a_{0}\left|\operatorname{Tr} J_{s-t}^{(k)} h_{\alpha}\left(x_{1}-x_{k+1}\right)\left(\gamma_{N, s}^{(k+1)}-\gamma_{\infty, s}^{(k+1)}\right)\right| \\
& \quad+8 \pi a_{0}\left|\operatorname{Tr} J_{s-t}^{(k)}\left[h_{\alpha}\left(x_{1}-x_{k+1}\right)-\delta\left(x_{1}-x_{k+1}\right)\right] \gamma_{\infty, s}^{(k+1)}\right| .
\end{aligned}
$$


Here we insert the wave operator $W_{N,(1, k+1)}$, because we only have a priori bounds on the quantity $W_{N,(1, k+1)}^{*} \gamma_{N, s}^{(k+1)}$. Then we replace $N V_{N}\left(x_{1}-x_{k+1}\right) W_{N,(1, k+1)}$ by $8 \pi a_{0} \delta\left(x_{1}-x_{k+1}\right)$. Afterwards, in order to remove the inverse wave operator $W_{N,(1, k+1)}^{*}$ and to take the limit $\gamma_{N, s}^{(k+1)} \rightarrow \gamma_{\infty, s}^{(k+1)}$, we need to replace the $\delta$-function by the bounded potential $h_{\alpha}$ independent of $N$. At the end, $h_{\alpha}$ is changed back to the $\delta$-function.

In Lemma 8.2 and Lemma 8.3 we prove that, for every $k \in \mathbb{N}$, for every $0 \leq s \leq$ $t \leq T$, and for every $J^{(k)} \in \mathcal{K}_{k}$ with (8.3),

$\left|\operatorname{Tr} J_{s-t}^{(k)}\left[N V_{N}\left(x_{1}-x_{k+1}\right) W_{N,(1, k+1)}-8 \pi a_{0} \delta\left(x_{1}-x_{k+1}\right)\right] W_{N,(1, k+1)}^{*} \gamma_{N, s}^{(k+1)}\right| \rightarrow 0$

as $N \rightarrow \infty$ and that

$$
\left|\operatorname{Tr} J_{s-t}^{(k)}\left[\delta\left(x_{1}-x_{k+1}\right)-h_{\alpha}\left(x_{1}-x_{k+1}\right)\right] W_{N,(1, k+1)}^{*} \gamma_{N, s}^{(k+1)}\right| \rightarrow 0
$$

as $\alpha \rightarrow 0$, uniformly in $N$.

As for the third term on the r.h.s. of (8.16) we remark that, for fixed $k \in \mathbb{N}$, $s \in[0, T], J^{(k)} \in \mathcal{K}_{k}$ and $\alpha>0$,

$$
\left|\operatorname{Tr} J_{s-t}^{(k)} h_{\alpha}\left(x_{1}-x_{k+1}\right)\left(W_{N,(1, k+1)}^{*}-1\right) \gamma_{N, s}^{(k+1)}\right| \rightarrow 0
$$

as $N \rightarrow \infty$. In fact, for the bounded operator $A=J_{s-t}^{(k)} h_{\alpha}\left(x_{1}-x_{k+1}\right)$, we can use the spectral decomposition $\gamma_{N, s}^{(k+1)}=\sum_{j} \lambda_{j}\left|\xi_{j}^{(k+1)}\right\rangle\left\langle\xi_{j}^{(k+1)}\right|$ with $\sum_{j} \lambda_{j}=1, \lambda_{j}>0$, $\left\|\xi_{j}^{(k+1)}\right\|=1$, and estimate

$$
\begin{aligned}
\left|\operatorname{Tr} A\left(W_{N,(1, k+1)}^{*}-1\right) \gamma_{N, s}^{(k+1)}\right| & \leq\|A\| \sum_{j} \lambda_{j}\left\|\left(W_{N,(1, k+1)}^{*}-1\right) \xi_{j}^{(k+1)}\right\|^{2} \\
& \leq C\|A\| N^{-1 / 3} \operatorname{Tr}\left(1-\Delta_{1}-\Delta_{k+1}\right) \gamma_{N, s}^{(k+1)} \\
& \leq C\|A\| N^{2 / 3}\left\langle\psi_{N, s},\left(H_{N}+N\right) \psi_{N, s}\right\rangle \leq C\|A\| N^{-1 / 3}
\end{aligned}
$$

by the energy conservation and (3.1). From the first to the second line we used Lemma 8.5. Since the operator $A$ is bounded for any fixed $J^{(k)}$ and $\alpha>0$, we obtain (8.19).

To control the fourth term on the r.h.s. of (8.16) we observe that, for arbitrary $\delta>0$

$$
\begin{aligned}
\operatorname{Tr} J_{s-t}^{(k)} & h_{\alpha}\left(x_{1}-x_{k+1}\right)\left(\gamma_{N, s}^{(k+1)}-\gamma_{\infty, s}^{(k+1)}\right) \\
= & \operatorname{Tr} J_{s-t}^{(k)} h_{\alpha}\left(x_{1}-x_{k+1}\right) \frac{1}{1+\delta\left(1-\Delta_{k+1}\right)^{1 / 2}}\left(\gamma_{N, s}^{(k+1)}-\gamma_{\infty, s}^{(k+1)}\right) \\
& \quad+\operatorname{Tr} J_{s-t}^{(k)} h_{\alpha}\left(x_{1}-x_{k+1}\right)\left(1-\frac{1}{1+\delta\left(1-\Delta_{k+1}\right)^{1 / 2}}\right)\left(\gamma_{N, s}^{(k+1)}-\gamma_{\infty, s}^{(k+1)}\right) .
\end{aligned}
$$

The first term on the r.h.s. of the last equation converges to zero, as $N \rightarrow \infty$, for every fixed $\delta, \alpha>0$. This follows from assumption (8.2) and from the observation that $J_{s-t}^{(k)} h_{\alpha}\left(x_{1}-x_{k+1}\right)\left(1+\delta\left(1-\Delta_{k+1}\right)\right)^{-1}$ is a compact operator on $L^{2}\left(\mathbb{R}^{3(k+1)}\right)$. 
As for the second term, we notice that it can be bounded by

$$
\begin{aligned}
& \left|\operatorname{Tr} J_{s-t}^{(k)} h_{\alpha}\left(x_{1}-x_{k+1}\right)\left(1-\frac{1}{1+\delta\left(1-\Delta_{k+1}\right)^{1 / 2}}\right)\left(\gamma_{N, s}^{(k+1)}-\gamma_{\infty, s}^{(k+1)}\right)\right| \\
& \quad \leq \delta\left\|J^{(k)}\right\|\left\|h_{\alpha}\right\|_{\infty} \operatorname{Tr}\left|\left(1-\Delta_{k+1}\right)^{1 / 2}\left(\gamma_{N, s}^{(k+1)}-\gamma_{\infty, s}^{(k+1)}\right)\right| \\
& \quad \leq \delta \alpha^{-3}\left\|J^{(k)}\right\|\|h\|_{\infty}\left(\operatorname{Tr}\left(1-\Delta_{k+1}\right) \gamma_{N, s}^{(k+1)}+\operatorname{Tr}\left(1-\Delta_{k+1}\right) \gamma_{\infty, s}^{(k+1)}\right) \\
& \quad \leq C \delta \alpha^{-3}
\end{aligned}
$$

uniformly in $N$. Choosing, for example, $\delta=\alpha^{4}$, it follows that

$$
\left|\operatorname{Tr} J_{s-t}^{(k)} h_{\alpha}\left(x_{1}-x_{k+1}\right)\left(\gamma_{N, s}^{(k+1)}-\gamma_{\infty, s}^{(k+1)}\right)\right| \leq \eta(\alpha, N)+C \alpha
$$

where $\eta(\alpha, N) \rightarrow 0$ as $N \rightarrow \infty$, for every fixed $\alpha>0$, and where the constant $C$ only depends on $J^{(k)}$.

Finally, using Lemma 10.3 and Theorem 7.3 , the last term on the r.h.s. of (8.16) can be controlled by

$$
\begin{aligned}
& \left|\operatorname{Tr} J_{s-t}^{(k)}\left[h_{\alpha}\left(x_{1}-x_{k+1}\right)-\delta\left(x_{1}-x_{k+1}\right)\right] \gamma_{\infty, s}^{(k+1)}\right| \\
& \quad \leq C \alpha^{1 / 2}\left\|J_{s-t}^{(k)}\right\| \operatorname{Tr}\left(1-\Delta_{1}\right)\left(1-\Delta_{k+1}\right) \gamma_{\infty, s}^{(k+1)} \\
& \quad \leq C\left(k, T, J^{(k)}\right) \alpha^{1 / 2} .
\end{aligned}
$$

From (8.16), (8.17), (8.18), (8.19), (8.23), and (8.24) it follows that, for every $k \geq 1,0 \leq s \leq t \leq T$, and $J^{(k)} \in \mathcal{K}_{k}$ with (8.3),

$$
\begin{aligned}
\mid \operatorname{Tr} J^{(k)} \mathcal{U}^{(k)}(t-s) N V_{N}\left(x_{1}-x_{k+1}\right) \gamma_{N, s}^{(k+1)} & \\
& -8 \pi a_{0} \operatorname{Tr} J^{(k)} \mathcal{U}^{(k)}(t-s) \delta\left(x_{1}-x_{k+1}\right) \gamma_{\infty, s}^{(k+1)} \mid \rightarrow 0
\end{aligned}
$$

as $N \rightarrow \infty$. Similarly to (8.25), we can also prove that

$$
\begin{aligned}
\mid \operatorname{Tr} J^{(k)} \mathcal{U}^{(k)}(t-s) \gamma_{N, s}^{(k+1)} & N V_{N}\left(x_{1}-x_{k+1}\right) \\
& -8 \pi a_{0} \operatorname{Tr} J^{(k)} \mathcal{U}^{(k)}(t-s) \gamma_{\infty, s}^{(k+1)} \delta\left(x_{1}-x_{k+1}\right) \mid \rightarrow 0
\end{aligned}
$$

as $N \rightarrow \infty$. Since (8.25) and (8.26) remain valid if we replace $x_{1}$ by any $x_{j}$, $j=2, \ldots, k$, in the potentials, it follows that, for every $k \geq 1,0 \leq s \leq t \leq T$, and $J^{(k)} \in \mathcal{K}_{k}$ with (8.3),

$$
\begin{aligned}
& \mid \sum_{j=1}^{k}\left(\operatorname{Tr} J^{(k)} \mathcal{U}^{(k)}(t-s)\right. {\left[N V_{N}\left(x_{j}-x_{k+1}\right), \gamma_{N, s}^{(k+1)}\right] } \\
&\left.-8 \pi a_{0} \operatorname{Tr} J^{(k)} \mathcal{U}^{(k)}(t-s)\left[\delta\left(x_{j}-x_{k+1}\right), \gamma_{\infty, s}^{(k+1)}\right]\right) \mid \rightarrow 0
\end{aligned}
$$

as $N \rightarrow \infty$. From (6.17) (with $J^{(k)}$ replaced by $\mathcal{U}^{(k)}(s-t) J^{(k)}$, using the fact that $\left\|\mathcal{U}^{(k)}(s-t) J^{(k)}\right\| \leq C_{T}$ for all $\left.0 \leq s \leq t \leq T\right)$ and from an estimate similar to (6.17) but with $\gamma_{N, s}^{(k+1)}$ replaced by $\gamma_{\infty, s}^{(k+1)}$ and $N V_{N}\left(x_{j}-x_{k+1}\right)$ replaced by $\delta\left(x_{j}-x_{k+1}\right)$, we can now apply the dominated convergence theorem to conclude (8.14). 
The following lemmas are important ingredients in the proof of Theorem 8.1 .

Lemma 8.2. Under the same assumptions as Theorem 8.1 and using the notation $J_{t}^{(k)}=\mathcal{U}^{(k)}(t) J^{(k)}$, we have, for every $k \geq 1, \ell=1, \ldots, k, 0 \leq s \leq t \leq T, J^{(k)} \in \mathcal{K}_{k}$ such that (8.3) is satisfied,

$$
\begin{aligned}
& \left|\operatorname{Tr} J_{s-t}^{(k)}\left[N V_{N}\left(x_{\ell}-x_{k+1}\right) W_{N,(\ell, k+1)}-8 \pi a_{0} \delta\left(x_{\ell}-x_{k+1}\right)\right] W_{N,(\ell, k+1)}^{*} \gamma_{N, s}^{(k+1)}\right| \rightarrow 0 \\
& \text { as } N \rightarrow \infty
\end{aligned}
$$

Proof. We fix $\ell=1$. Decomposing $\gamma_{N, s}^{(k+1)}=\sum_{j} \lambda_{j}\left|\xi_{j}^{(k+1)}\right\rangle\left\langle\xi_{j}^{(k+1)}\right|$ and introducing the variables $u=\left(x_{1}+x_{k+1}\right) / 2$ and $v=x_{1}-x_{k+1}$, we find

$$
\begin{aligned}
\operatorname{Tr} J_{s-t}^{(k)} & N V_{N}\left(x_{1}-x_{k+1}\right) \gamma_{N, s}^{(k+1)} \\
= & \sum_{j} \lambda_{j} \int \mathrm{d} u \mathrm{~d} v \mathrm{~d} x_{2} \ldots \mathrm{d} x_{k} \mathrm{~d} \mathbf{x}_{k}^{\prime} J_{s-t}^{(k)}\left(\mathbf{x}_{k}^{\prime} ; u+v / 2, x_{2}, \ldots, x_{k}\right) N V_{N}(v) \\
& \times \xi_{j}^{(k+1)}\left(u+v / 2, x_{2}, \ldots, x_{k}, u-v / 2\right) \bar{\xi}_{j}^{(k+1)}\left(\mathbf{x}_{k}^{\prime}, u-v / 2\right) .
\end{aligned}
$$

The potential $V_{N}(v)$ forces $v$ to be of order $1 / N$. Using this fact, we are going to remove the $v$-dependence from the observable and from the wave function $\bar{\xi}_{j}^{(k+1)}$. After removing this $v$-dependence, we introduce the wave operator using its $L^{2}$ unitarity. We find

$$
\begin{aligned}
& \operatorname{Tr} J_{s-t}^{(k)} N V_{N}\left(x_{1}-x_{k+1}\right) \gamma_{N, s}^{(k+1)} \\
& =\sum_{j} \lambda_{j} \int \mathrm{d} u \mathrm{~d} v \mathrm{~d} x_{2} \ldots \mathrm{d} x_{k} \mathrm{~d} \mathbf{x}_{k}^{\prime} N V_{N}(v) \xi_{j}^{(k+1)}\left(u+v / 2, x_{2}, \ldots, x_{k}, u-v / 2\right) \\
& \quad \times\left[J_{s-t}^{(k)}\left(\mathbf{x}_{k}^{\prime} ; u+v / 2, x_{2}, \ldots, x_{k}\right) \bar{\xi}_{j}^{(k+1)}\left(\mathbf{x}_{k}^{\prime}, u-v / 2\right)\right. \\
& \left.\quad-J_{s-t}^{(k)}\left(\mathbf{x}_{k}^{\prime} ; u, x_{2}, \ldots, x_{k}\right) \bar{\xi}_{j}^{(k+1)}\left(\mathbf{x}_{k}^{\prime}, u\right)\right] \\
& +\sum_{j} \lambda_{j} \int \mathrm{d} u \mathrm{~d} v \mathrm{~d} x_{2} \ldots \mathrm{d} x_{k} \mathrm{~d} \mathbf{x}_{k}^{\prime} N\left(W_{N}^{*} V_{N}\right)(v) J_{s-t}^{(k)}\left(\mathbf{x}_{k}^{\prime} ; u, x_{2}, \ldots, x_{k}\right) \bar{\xi}_{j}^{(k+1)}\left(\mathbf{x}_{k}^{\prime}, u\right) \\
& \quad \times\left[\left(W_{N,(1, k+1)}^{*} \xi_{j}^{(k+1)}\right)\left(u+v / 2, x_{2}, \ldots, x_{k}, u-v / 2\right)\right. \\
& \left.\quad-\left(W_{N,(1, k+1)}^{*} \xi_{j}^{(k+1)}\right)\left(u, x_{2}, \ldots, x_{k}, u\right)\right] \\
& +\sum_{j} \lambda_{j}\left(\int \mathrm{d} v\left(W_{N}^{*} V_{N}\right)(v)\right) \int \mathrm{d} u \mathrm{~d} x_{2} \ldots \mathrm{d} x_{k} \mathrm{~d} \mathbf{x}_{k}^{\prime} J_{s-t}^{(k)}\left(\mathbf{x}_{k}^{\prime} ; u, x_{2}, \ldots, x_{k}\right) \\
& \quad \times \bar{\xi}_{j}^{(k+1)}\left(\mathbf{x}_{k}^{\prime}, u\right)\left(W_{N,(1, k+1)}^{*} \xi_{j}^{(k+1)}\right)\left(u, x_{2}, \ldots, x_{k}, u\right) .
\end{aligned}
$$


From Lemma 8.4, we know that

$$
\int \mathrm{d} v N\left(W_{N}^{*} V_{N}\right)(v)=8 \pi a_{0} .
$$

Therefore, from (8.29), we obtain that

$$
\begin{aligned}
& \left|\operatorname{Tr} J_{s-t}^{(k)}\left[N V_{N}\left(x_{1}-x_{k+1}\right) W_{N,(1, k+1)}-8 \pi a_{0} \delta\left(x_{1}-x_{k+1}\right)\right] W_{N,(1, k+1)}^{*} \gamma_{N, s}^{(k+1)}\right| \\
& \leq \sum_{j} \lambda_{j} \int \mathrm{d} u \mathrm{~d} v \mathrm{~d} x_{2} \ldots \mathrm{d} x_{k} \mathrm{~d} \mathbf{x}_{k}^{\prime} N V_{N}(v)\left|\xi_{j}^{(k+1)}\left(u+v / 2, x_{2}, \ldots, x_{k}, u-v / 2\right)\right| \\
& \times \mid J_{s-t}^{(k)}\left(\mathbf{x}_{k}^{\prime} ; u+v / 2, x_{2}, \ldots, x_{k}\right) \bar{\xi}_{j}^{(k+1)}\left(\mathbf{x}_{k}^{\prime}, u-v / 2\right) \\
& -J_{s-t}^{(k)}\left(\mathbf{x}_{k}^{\prime} ; u, x_{2}, \ldots, x_{k}\right) \bar{\xi}_{j}^{(k+1)}\left(\mathbf{x}_{k}^{\prime}, u\right) \\
& +\sum_{j} \lambda_{j} \mid \int \mathrm{d} u \mathrm{~d} v \mathrm{~d} x_{2} \ldots \mathrm{d} x_{k} \mathrm{~d} \mathbf{x}_{k}^{\prime} N\left(W_{N}^{*} V_{N}\right)(v) J_{s-t}^{(k)}\left(\mathbf{x}_{k}^{\prime} ; u, x_{2}, \ldots, x_{k}\right) \bar{\xi}_{j}^{(k+1)}\left(\mathbf{x}_{k}^{\prime}, u\right) \\
& \times\left[\left(W_{N,(1, k+1)}^{*} \xi_{j}^{(k+1)}\right)\left(u+v / 2, x_{2}, \ldots, x_{k}, u-v / 2\right)\right. \\
& \left.-\left(W_{N,(1, k+1)}^{*} \xi_{j}^{(k+1)}\right)\left(u, x_{2}, \ldots, x_{k}, u\right)\right] \mid \\
& =: \sum_{j} \lambda_{j}\left(\mathrm{I}_{j}+\mathrm{II}_{j}\right)
\end{aligned}
$$

The terms $\mathrm{I}_{j}$ can be bounded exactly like the term I on the r.h.s. of (6.14), after replacing $J^{(k)}$ by $J_{s-t}^{(k)}$. Following the steps (6.15)-(6.16), we find that

$$
\sum_{j} \lambda_{j} \mathrm{I}_{j} \leq \frac{C_{k}\left\|J_{s-t}^{(k)}\right\|}{N^{1 / 2}} \operatorname{Tr}\left(-\Delta_{1}-\Delta_{k+1}+1\right) W_{N,(1, k+1)}^{*} \gamma_{N, s}^{(k+1)} W_{N,(1, k+1)},
$$

which converges to zero as $N \rightarrow \infty$ for all $0 \leq s \leq t \leq T$ and all observables $J^{(k)}$ satisfying (8.3) (here we used the a priori estimate given in Proposition 5.3 and the observation (8.6) ).

Next, we consider the second term on the r.h.s. of (8.30):

$$
\begin{aligned}
\mathrm{II}_{j}= & \mid \int \mathrm{d} u \mathrm{~d} v \mathrm{~d} x_{2} \ldots \mathrm{d} x_{k} \mathrm{~d} \mathbf{x}_{k}^{\prime} J_{s-t}^{(k)}\left(\mathbf{x}_{k}^{\prime} ; u, x_{2}, \ldots, x_{k}\right) \bar{\xi}_{j}^{(k+1)}\left(\mathbf{x}_{k}^{\prime}, u\right) \\
& \times\left(N^{3}\left(W^{*} V\right)(N v)-\delta(v)\right)\left(W_{N,(1, k+1)}^{*} \xi_{j}^{(k+1)}\right)\left(u+v / 2, x_{2}, \ldots, x_{k}, u-v / 2\right) \mid .
\end{aligned}
$$

To control this contribution, we first insert a cutoff $\chi(v)$; this will allow us to apply Lemma 10.2 to bound the integral over $u$ and $v$. To this end, we choose a function $\chi \in C_{0}^{\infty}\left(\mathbb{R}^{3}\right)$ such that $0 \leq \chi(x) \leq 1, \chi(x)=1$ for $|x| \leq 1$ and $\chi(x)=0$ for $|x| \geq 2$, 
and we put $\bar{\chi}=1-\chi$. Using $\chi$, we decompose the r.h.s. of (8.32) in two parts:

$$
\begin{aligned}
\mathrm{II}_{j} \leq \mid & \int \mathrm{d} u \mathrm{~d} v \mathrm{~d} x_{2} \ldots \mathrm{d} x_{k} \mathrm{~d} \mathbf{x}_{k}^{\prime} J_{s-t}^{(k)}\left(\mathbf{x}_{k}^{\prime} ; u, x_{2}, \ldots, x_{k}\right) \chi(v) \bar{\xi}_{j}^{(k+1)}\left(\mathbf{x}_{k}^{\prime}, u\right) \\
\times & {\left[N^{3}\left(W^{*} V\right)(N v)-\delta(v)\right]\left(W_{N,(1, k+1)}^{*} \xi_{j}^{(k+1)}\right)\left(u+v / 2, x_{2}, \ldots, x_{k}, u-v / 2\right) \mid } \\
+ & \mid \int \mathrm{d} u \mathrm{~d} v \mathrm{~d} x_{2} \ldots \mathrm{d} x_{k} \mathrm{~d} \mathbf{x}_{k}^{\prime} J_{s-t}^{(k)}\left(\mathbf{x}_{k}^{\prime} ; u, x_{2}, \ldots, x_{k}\right) \bar{\chi}(v) \bar{\xi}_{j}^{(k+1)}\left(\mathbf{x}_{k}^{\prime}, u\right) \\
& \times N^{3}\left(W^{*} V\right)(N v)\left(W_{N,(1, k+1)}^{*} \xi_{j}^{(k+1)}\right)\left(u+v / 2, x_{2}, \ldots, x_{k}, u-v / 2\right) \mid \\
=: & \mathrm{A}_{j}+\mathrm{B}_{j} .
\end{aligned}
$$

The term $\mathrm{B}_{j}$ can be bounded by

$$
\begin{aligned}
\mathrm{B}_{j} \leq \int \mathrm{d} u \mathrm{~d} v \mathrm{~d} x_{2} \ldots \mathrm{d} x_{k} \mathrm{~d} \mathbf{x}_{k}^{\prime}\left|J_{s-t}^{(k)}\left(\mathbf{x}_{k}^{\prime} ; u, x_{2}, \ldots, x_{k}\right)\right| \bar{\chi}(v) N^{3}\left(W^{*} V\right)(N v) \\
\quad \times\left(\left|\xi_{j}^{(k+1)}\left(\mathbf{x}_{k}^{\prime}, u\right)\right|^{2}+\left|\left(W_{N,(1, k+1)}^{*} \xi_{j}^{(k+1)}\right)\left(u+v / 2, x_{2}, \ldots, x_{k}, u-v / 2\right)\right|^{2}\right) \\
\leq\left\|\xi_{j}^{(k+1)}\right\|^{2}\left(\sup _{u, \mathbf{x}_{k}^{\prime}} \int \mathrm{d} x_{2} \ldots \mathrm{d} x_{k}\left|J_{s-t}^{(k)}\left(\mathbf{x}_{k}^{\prime} ; u, x_{2}, \ldots, x_{k}\right)\right|\right) \int_{|v| \geq N}\left|\left(W^{*} V\right)(v)\right| \mathrm{d} v \\
\left.+\quad \sup _{\mathbf{x}_{k}} \int \mathrm{d} \mathbf{x}_{k}^{\prime}\left|J_{s-t}^{(k)}\left(\mathbf{x}_{k}^{\prime} ; \mathbf{x}_{k}\right)\right|\right) \\
\quad \times \int \mathrm{d} x_{1} \ldots \mathrm{d} x_{k+1} \bar{\chi}\left(x_{1}-x_{k+1}\right) N^{3}\left(W^{*} V\right)\left(N\left(x_{1}-x_{k+1}\right)\right) \\
\quad \times\left|\left(W_{N,(1, k+1)}^{*} \xi_{j}^{(k+1)}\right)\left(\mathbf{x}_{k}, x_{k+1}\right)\right|^{2} .
\end{aligned}
$$

From Lemma 10.1, we obtain

$$
\begin{aligned}
& \sum_{j} \lambda_{j} \mathrm{~B}_{j} \leq C\left(k, T, J^{(k)}\right)\left(\int_{|v| \geq N} \mathrm{~d} v\left|\left(W^{*} V\right)(v)\right|\right) \\
& \quad \times\left(\operatorname{Tr}\left(\left(\nabla_{1} \cdot \nabla_{2}\right)^{2}-\Delta_{1}-\Delta_{2}+1\right) W_{N,(1, k+1)} \gamma_{N, s}^{(k+1)} W_{N,(1, k+1)}^{*}\right) \rightarrow 0
\end{aligned}
$$

as $N \rightarrow \infty$. Here we used Proposition 5.3 and the fact that, since $W^{*} V \in L^{1}\left(\mathbb{R}^{3}\right)$,

$$
\int_{|x|>N}\left|W^{*} V(x)\right| \mathrm{d} x \rightarrow 0 \quad \text { as } \quad N \rightarrow \infty
$$

As for the term $\mathrm{A}_{j}$ on the r.h.s. of (8.33), Lemma 10.2 implies that there exists a sequence $\delta_{N} \rightarrow 0$ as $N \rightarrow \infty$ ( $\delta_{N}$ corresponds to the sequence $\beta_{1 / N}$ defined in 
Lemma 10.2. with $V$ replaced by $W^{*} V$ ) such that

$$
\begin{aligned}
\mathrm{A}_{j} \leq & \delta_{N} \int \mathrm{d} x_{2} \ldots \mathrm{d} x_{k} \mathrm{~d} \mathbf{x}_{k}^{\prime} \\
& \times\left(\int \mathrm{d} u \mathrm{~d} v \mid\left(\left(\Delta_{u}-\Delta_{v}\right)^{2}-\Delta_{u}-\Delta_{v}+1\right)^{1 / 2}\right. \\
& \left.\times\left.\left(W_{N,(1, k+1)}^{*} \xi_{j}^{(k+1)}\right)\left(u+v / 2, x_{2}, \ldots, x_{k}, u-v / 2\right)\right|^{2}\right)^{1 / 2} \\
& \times\left(\int \mathrm{d} u \mathrm{~d} v\left|\left(1-\Delta_{u}+\Delta_{v}^{2}\right)^{1 / 2} \chi(v) J_{s-t}^{(k)}\left(\mathbf{x}_{k}^{\prime} ; u, x_{2}, \ldots, x_{k}\right) \bar{\xi}_{j}^{(k+1)}\left(\mathbf{x}_{k}^{\prime}, u\right)\right|^{2}\right)^{1 / 2} \\
\leq & \delta_{N}\|\chi\|_{H^{2}} \int \mathrm{d} x_{2} \ldots \mathrm{d} x_{k} \mathrm{~d} \mathbf{x}_{k}^{\prime}\left(\int \mathrm{d} u\left|\left(1-\Delta_{u}\right)^{1 / 2} \bar{\xi}_{j}^{(k+1)}\left(\mathbf{x}_{k}^{\prime}, u\right)\right|^{2}\right)^{1 / 2} \\
& \times \sup _{u}\left[\left|J_{s-t}^{(k)}\left(\mathbf{x}_{k}^{\prime} ; u, x_{2}, \ldots, x_{k}\right)\right|+\left|\nabla_{u} J_{s-t}^{(k)}\left(\mathbf{x}_{k}^{\prime} ; u, x_{2}, \ldots, x_{k}\right)\right|\right] \\
& \times\left(\int \mathrm{d} u \mathrm{~d} v \mid\left(\left(\Delta_{u}-\Delta_{v}\right)^{2}-\Delta_{u}-\Delta_{v}+1\right)^{1 / 2}\right. \\
& \left.\times\left.\left(W_{N,(1, k+1)}^{*} \xi_{j}^{(k+1)}\right)\left(u+v / 2, x_{2}, \ldots, x_{k}, u-v / 2\right)\right|^{2}\right)^{1 / 2} .
\end{aligned}
$$

With a Schwarz inequality, we find

$$
\begin{aligned}
\mathrm{A}_{j} \leq & \delta_{N}\|\chi\|_{H^{2}} \int \mathrm{d} x_{2} \ldots \mathrm{d} x_{k} \mathrm{~d} \mathbf{x}_{k}^{\prime} \\
& \times \sup _{u}\left[\left|J_{s-t}^{(k)}\left(\mathbf{x}_{k}^{\prime} ; u, x_{2}, \ldots, x_{k}\right)\right|+\left|\nabla_{u} J_{s-t}^{(k)}\left(\mathbf{x}_{k}^{\prime} ; u, x_{2}, \ldots, x_{k}\right)\right|\right] \\
& \times\left(\int \mathrm{d} u\left|\left(1-\Delta_{u}\right)^{1 / 2} \bar{\xi}_{j}^{(k+1)}\left(\mathbf{x}_{k}^{\prime}, u\right)\right|^{2}\right. \\
& +\int \mathrm{d} u \mathrm{~d} v \mid\left(\left(\Delta_{u}-\Delta_{v}\right)^{2}-\Delta_{u}-\Delta_{v}+1\right)^{1 / 2} \\
& \left.\times\left.\left(W_{N,(1, k+1)}^{*} \xi_{j}^{(k+1)}\right)\left(u+v / 2, x_{2}, \ldots, x_{k}, u-v / 2\right)\right|^{2}\right) \\
\leq & C\left(k, T, J^{(k)}\right) \delta_{N}\left(\left\langle\xi_{j}^{(k+1)},\left(1-\Delta_{u}\right) \xi_{j}^{(k+1)}\right\rangle\right. \\
& \left.+\left\langle W_{N,(1, k+1)}^{*} \xi_{j}^{(k+1)},\left(\left(\nabla_{1} \cdot \nabla_{k+1}\right)^{2}-\Delta_{1}-\Delta_{k+1}+1\right) W_{N,(1, k+1)}^{*} \xi_{j}^{(k+1)}\right\rangle\right) .
\end{aligned}
$$

From Lemma 10.2 and Proposition 5.3, we find

$$
\sum_{j} \lambda_{j} \mathrm{~A}_{j} \leq C \delta_{N} \rightarrow 0 \quad \text { as } N \rightarrow \infty,
$$

and this, with (8.34), implies that

$$
\sum_{j} \lambda_{j} \mathrm{II}_{j} \rightarrow 0 \quad \text { as } N \rightarrow \infty
$$

Together with (8.31) and (8.30), this concludes the proof of the lemma. 
Lemma 8.3. Under the same conditions as in Theorem 8.1, we have, for every $k \geq 1, \ell=1, \ldots, k, 0 \leq s \leq T$, and $J^{(k)} \in \mathcal{K}_{k}$ satisfying (8.3),

$$
\left|\operatorname{Tr} J_{s-t}^{(k)}\left[\delta\left(x_{\ell}-x_{k+1}\right)-h_{\alpha}\left(x_{\ell}-x_{k+1}\right)\right] W_{N,(\ell, k+1)}^{*} \gamma_{N, s}^{(k+1)}\right| \rightarrow 0
$$

as $\alpha \rightarrow 0$, uniformly in $N$. Here we use the notation $J_{t}^{(k)}=\mathcal{U}^{(k)}(t) J^{(k)}$.

Proof. We fix $\ell=1$. Using the decomposition $\gamma_{N, s}^{(k+1)}=\sum_{j} \lambda_{j}\left|\xi_{j}^{(k+1)}\right\rangle\left\langle\xi_{j}^{(k+1)}\right|$, we find that

$$
\begin{aligned}
& \left|\operatorname{Tr} J_{s-t}^{(k)}\left[\delta\left(x_{1}-x_{k+1}\right)-h_{\alpha}\left(x_{1}-x_{k+1}\right)\right] W_{N,(1, k+1)}^{*} \gamma_{N, s}^{(k+1)}\right| \\
& \leq \sum_{j} \lambda_{j} \mid \int \mathrm{d} u \mathrm{~d} v \mathrm{~d} x_{2} \ldots \mathrm{d} x_{k} \mathrm{~d} \mathbf{x}_{k}^{\prime} J_{s-t}^{(k)}\left(\mathbf{x}_{k}^{\prime} ; u+v / 2, x_{2}, \ldots, x_{k}\right)\left[\delta(v)-h_{\alpha}(v)\right] \\
& \quad \times \bar{\xi}_{j}^{(k+1)}\left(\mathbf{x}_{k}^{\prime}, u-v / 2\right)\left(W_{N,(1, k+1)}^{*} \xi_{j}^{(k+1)}\right)\left(u+v / 2, x_{2}, \ldots, x_{k}, u-v / 2\right) \mid \\
& \leq \sum_{j} \lambda_{j} \mid \int \mathrm{d} u \mathrm{~d} v \mathrm{~d} x_{2} \ldots \mathrm{d} x_{k} \mathrm{~d} \mathbf{x}_{k}^{\prime} h_{\alpha}(v) \\
& \quad \times\left[J_{s-t}^{(k)}\left(\mathbf{x}_{k}^{\prime} ; u+v / 2, x_{2}, \ldots, x_{k}\right) \bar{\xi}_{j}^{(k+1)}\left(\mathbf{x}_{k}^{\prime}, u-v / 2\right)\right. \\
& \quad \times\left(W_{N,(1, k+1)}^{*} \xi_{j}^{(k+1)}\right)\left(u+v / 2, x_{2}, \ldots, x_{k}, u-v / 2\right) \\
& \left.\quad-J_{s-t}^{(k)}\left(\mathbf{x}_{k}^{\prime} ; u, x_{2}, \ldots, x_{k}\right) \bar{\xi}_{j}^{(k+1)}\left(\mathbf{x}_{k}^{\prime}, u\right)\left(W_{N,(1, k+1)}^{*} \xi_{j}^{(k+1)}\right)\left(u, x_{2}, \ldots, x_{k}, u\right)\right] \mid .
\end{aligned}
$$

Similarly to (8.29), we first replace $v$ by 0 in $J_{s-t}^{(k)}\left(\mathbf{x}_{k}^{\prime} ; u+v / 2, x_{2}, \ldots, x_{k}\right) \bar{\xi}_{j}^{(k+1)}\left(\mathbf{x}_{k}^{\prime}\right.$, $u-v / 2)$ and then in $\left(W_{N,(1, k+1)}^{*} \xi_{j}^{(k+1)}\right)\left(u+v / 2, x_{2}, \ldots, x_{k}, u-v / 2\right)$. We obtain

$$
\begin{aligned}
& \left|\operatorname{Tr} J_{s-t}^{(k)}\left[\delta\left(x_{1}-x_{k+1}\right)-h_{\alpha}\left(x_{1}-x_{k+1}\right)\right] W_{N,(1, k+1)}^{*} \gamma_{N, s}^{(k+1)}\right| \\
& \leq \sum_{j} \lambda_{j} \mid \int \mathrm{d} u \mathrm{~d} v \mathrm{~d} x_{2} \ldots \mathrm{d} x_{k} \mathrm{~d} \mathbf{x}_{k}^{\prime} h_{\alpha}(v) \\
& \times\left(W_{N,(1, k+1)}^{*} \xi_{j}^{(k+1)}\right)\left(u+v / 2, x_{2}, \ldots, x_{k}, u-v / 2\right) \\
& \times\left[J_{s-t}^{(k)}\left(\mathbf{x}_{k}^{\prime} ; u+v / 2, x_{2}, \ldots, x_{k}\right) \bar{\xi}_{j}^{(k+1)}\left(\mathbf{x}_{k}^{\prime}, u-v / 2\right)\right. \\
& \left.-J_{s-t}^{(k)}\left(\mathbf{x}_{k}^{\prime} ; u, x_{2}, \ldots, x_{k}\right) \bar{\xi}_{j}^{(k+1)}\left(\mathbf{x}_{k}^{\prime}, u\right)\right] \mid \\
& +\sum_{j} \lambda_{j} \mid \int \mathrm{d} u \mathrm{~d} v \mathrm{~d} x_{2} \ldots \mathrm{d} x_{k} \mathrm{~d} \mathbf{x}_{k}^{\prime} h_{\alpha}(v) J_{s-t}^{(k)}\left(\mathbf{x}_{k}^{\prime} ; u, x_{2}, \ldots, x_{k}\right) \bar{\xi}_{j}^{(k+1)}\left(\mathbf{x}_{k}^{\prime}, u\right) \\
& \times\left[\left(W_{N,(1, k+1)}^{*} \xi_{j}^{(k+1)}\right)\left(u+v / 2, x_{2}, \ldots, x_{k}, u-v / 2\right)\right. \\
& \left.-\left(W_{N,(1, k+1)}^{*} \xi_{j}^{(k+1)}\right)\left(u, x_{2}, \ldots, x_{k}, u\right)\right] \mid \\
& =: \sum_{j} \lambda_{j}\left(\mathrm{III}_{j}+\mathrm{IV}_{j}\right)
\end{aligned}
$$


To bound the first term, we expand the difference in an integral

$$
\begin{aligned}
& {\left[J_{s-t}^{(k)}\left(\mathbf{x}_{k}^{\prime} ; u+v / 2, x_{2}, \ldots, x_{k}\right) \bar{\xi}_{j}^{(k+1)}\left(\mathbf{x}_{k}^{\prime}, u-v / 2\right)\right.} \\
& \left.-J_{s-t}^{(k)}\left(\mathbf{x}_{k}^{\prime} ; u, x_{2}, \ldots, x_{k}\right) \bar{\xi}_{j}^{(k+1)}\left(\mathbf{x}_{k}^{\prime}, u\right)\right] \\
& =\int_{0}^{1 / 2} \mathrm{~d} r v \cdot \nabla_{1} J_{s-t}^{(k)}\left(\mathbf{x}_{k}^{\prime} ; u+r v, x_{2}, \ldots, x_{k}\right) \bar{\xi}_{j}^{(k+1)}\left(\mathbf{x}_{k}^{\prime}, u-r v\right) \\
& \quad-\int_{0}^{1 / 2} \mathrm{~d} r J_{s-t}^{(k)}\left(\mathbf{x}_{k}^{\prime} ; u+r v, x_{2}, \ldots, x_{k}\right) v \cdot \nabla_{k+1} \bar{\xi}_{j}^{(k+1)}\left(\mathbf{x}_{k}^{\prime}, u-r v\right)
\end{aligned}
$$

and we obtain that

$$
\begin{aligned}
& \operatorname{III}_{j} \leq \int \mathrm{d} u \mathrm{~d} v \mathrm{~d} x_{2} \ldots \mathrm{d} x_{k} \mathrm{~d} \mathbf{x}_{k}^{\prime} \int_{0}^{1 / 2} \mathrm{~d} r h_{\alpha}(v)|v| \\
& \times\left|\left(W_{N}^{*} \xi_{j}^{(k+1)}\right)\left(u+v / 2, x_{2}, \ldots, x_{k}, u-v / 2\right)\right| \\
& \times\left(\left|\nabla_{1} J_{s-t}^{(k)}\left(\mathbf{x}_{k}^{\prime} ; u+r v, x_{2}, \ldots, x_{k}\right)\right|\left|\xi_{j}^{(k+1)}\left(\mathbf{x}_{k}^{\prime}, u-r v\right)\right|\right. \\
&\left.+\left|J_{s-t}^{(k)}\left(\mathbf{x}_{k}^{\prime} ; u+r v, x_{2}, \ldots, x_{k}\right)\right|\left|\nabla_{k+1} \xi_{j}^{(k+1)}\left(\mathbf{x}_{k}^{\prime}, u-r v\right)\right|\right),
\end{aligned}
$$

which implies that

$$
\begin{aligned}
\sum_{j} \lambda_{j} \mathrm{III}_{j} \leq \alpha C\left(k, T, J^{(k)}\right)( & \operatorname{Tr}\left(1-\Delta_{k+1}\right) \gamma_{N, s}^{(k+1)} \\
& \left.+\operatorname{Tr}\left(\left(\nabla_{1} \cdot \nabla_{k+1}\right)^{2}-\Delta_{1}-\Delta_{k+1}+1\right) \gamma_{N, s}^{(k+1)}\right) \\
\leq C \alpha . &
\end{aligned}
$$

The terms $\mathrm{IV}_{j}$ can be estimated similarly to the terms $\mathrm{II}_{j}$ considered in (8.32). In particular, analogously to (8.34) and (8.37), we also find

$$
\begin{aligned}
\sum_{j} \lambda_{j} \mathrm{IV}_{j} \leq C\left(k, T, J^{(k)}\right) \beta_{\alpha}( & \operatorname{Tr}\left(1-\Delta_{k+1}\right) \gamma_{N, s}^{(k+1)} \\
& \left.+\operatorname{Tr}\left(\left(\nabla_{1} \cdot \nabla_{k+1}\right)^{2}-\Delta_{1}-\Delta_{k+1}+1\right) \gamma_{N, s}^{(k+1)}\right) \\
\leq C \beta_{\alpha}, &
\end{aligned}
$$

where $\beta_{\alpha} \rightarrow 0$ as $\alpha \rightarrow 0$ uniformly in $N$ (the sequence $\beta_{\alpha}$ comes from Lemma 10.2 . with $V$ replaced by $h$ ). This concludes the proof of the lemma.

Lemma 8.4. Suppose that $V \geq 0$, with $V(x) \leq C\langle x\rangle^{-\sigma}$ for some $\sigma>5$ (this implies, in particular, that $V \in L^{1}\left(\mathbb{R}^{3}\right) \cap L^{2}\left(\mathbb{R}^{3}\right)$ and thus that $V$ is in the Rollnik class of potentials). Let $W$ denote the wave operator (as defined in Proposition 5.1) associated with the Hamiltonian $\mathfrak{h}=-\Delta+(1 / 2) V(x)$. Then

$$
\int \mathrm{d} x\left(W^{*} V\right)(x)=8 \pi a_{0},
$$

where $a_{0}$ is the scattering length of the potential $V$.

Proof. First of all, we observe that, under the assumption that $V \geq 0$ and $V(x) \leq$ $C\langle x\rangle^{-\sigma}$, for some $\sigma>5$, the operator $\mathfrak{h}=-\Delta+(1 / 2) V$ cannot have a zero energy resonance (recall that a zero-energy resonance of $\mathfrak{h}$ is a solution $\varphi$ of $(-\Delta+$ 
$(1 / 2) V) \varphi=0$ such that $|\varphi(x)| \leq C /|x|$ for all $\left.x \in \mathbb{R}^{3}\right)$; this can be proven using the maximum principle. We will make use of this observation in the proof of this lemma.

Next, we note that, since $W^{*}$ maps $L^{1}\left(\mathbb{R}^{3}\right)$ into $L^{1}\left(\mathbb{R}^{3}\right)$ (see Proposition 5.1), we have that $\left(W^{*} V\right) \in L^{1}\left(\mathbb{R}^{3}\right)$, and thus

$$
\int \mathrm{d} x\left(W^{*} V\right)(x)=\lim _{\varepsilon \rightarrow 0} \int \mathrm{d} x\left(W^{*} V\right)(x) \chi_{\varepsilon}(x)=\lim _{\varepsilon \rightarrow 0} \int \mathrm{d} x V(x)\left(W \chi_{\varepsilon}\right)(x)
$$

with

$$
\chi_{\varepsilon}(x)=\frac{1}{1+\varepsilon x^{2}} .
$$

We expand $W \chi_{\varepsilon}$ in terms of solutions $\varphi(x, k)$ of the Lippman-Schwinger equation

$$
\varphi(x, k)=e^{i k \cdot x}-\frac{1}{8 \pi} \int \mathrm{d} y \frac{e^{i|k||x-y|}}{|x-y|} V(y) \varphi(y, k) .
$$

It follows from [24, Theorem XI.41, a)] that Eq. (8.42) has a unique solution $\varphi(x, k)$, such that $\varphi(x, k) V^{1 / 2}(x) \in L^{2}\left(\mathbb{R}^{3}\right)$, for all $k \in \mathbb{R}^{3}$ such that $k^{2} \notin \mathcal{E}$, for an exceptional set $\mathcal{E}$ with Lebesgue measure zero. The set $\mathcal{E}$ consists of all values of $k^{2}$ for which zero is an eigenvalue of the operator

$$
M_{|k|}=1+\frac{1}{2} V^{1 / 2} \frac{1}{-\Delta-k^{2}} V^{1 / 2} .
$$

From the observation that the operator $\mathfrak{h}=-\Delta+(1 / 2) V$ does not have a zero energy resonance, it follows immediately that $0 \notin \mathcal{E}$. In fact, if $M_{0} \psi=0$ for some $\psi \in L^{2}\left(\mathbb{R}^{3}\right)$, then

$$
\psi(x)=-\frac{1}{2} V^{1 / 2}(x) \int \mathrm{d} y \frac{1}{|x-y|} V^{1 / 2}(y) \psi(y),
$$

which implies that $\psi(x) / V^{1 / 2}(x) \leq C /|x|$ for $|x| \gg 1$ and thus that $\varphi(x):=$ $\psi(x) / V^{1 / 2}(x)$ is a zero-energy resonance solution of $(-\Delta+(1 / 2) V) \varphi=0$. Since $M_{0}$ is a nonnegative Fredholm operator with no eigenvalue at zero, it follows that there exists $\lambda>0$ with $\sigma\left(M_{0}\right) \subset(\lambda, \infty)$ (here $\sigma\left(M_{0}\right)$ indicates the spectrum of $\left.M_{0}\right)$. Since moreover $M_{|k|}-M_{0}$ is a compact operator with kernel

$$
\left(M_{|k|}-M_{0}\right)(x ; y)=\frac{1}{2} V^{1 / 2}(x) \frac{e^{i|k||x-y|}-1}{|x-y|} V^{1 / 2}(y),
$$

we obtain that

$$
\left\|M_{|k|}-M_{0}\right\|_{\mathrm{HS}}^{2}=\frac{1}{4} \int \mathrm{d} x \mathrm{~d} y V(x) V(y) \frac{\left|e^{i|k||x-y|}-1\right|^{2}}{|x-y|^{2}} \leq \frac{|k|^{2}\|V\|_{L^{1}}^{2}}{4}
$$

and thus that there exists $\kappa>0$ such that $\sigma\left(M_{|k|}\right) \subset(\lambda / 2, \infty)$ for all $|k| \leq \kappa$. In particular it follows that

$$
\left\|M_{|k|}^{-1}\right\| \leq 2 / \lambda \quad \text { for all } k \in \mathbb{R}^{3} \text { with }|k| \leq \kappa .
$$

From [24, Theorem XI.41, e)] we also find

$$
\left(W \chi_{\varepsilon}\right)(x)=\text { L.I.M. }(2 \pi)^{-3 / 2} \int \mathrm{d} k \varphi(x, k) \widehat{\chi}_{\varepsilon}(k)=\text { L.I.M. } \int \mathrm{d} k \varphi(x, k) \frac{e^{-|k| / \sqrt{\varepsilon}}}{4 \pi|k| \varepsilon},
$$


where L.I.M. denotes the $L^{2}$-limit as $M \rightarrow \infty$ and $\delta \rightarrow 0$ of the integral over $\left\{k \in \mathbb{R}^{3}:|k| \leq M\right.$ and dist $\left.\left(k^{2}, \mathcal{E}\right)>\delta\right\}$. Inserting (8.47) on the r.h.s. of (8.41), we find (recalling that $\kappa>0$ is chosen such that (8.46) holds true)

$$
\begin{aligned}
\int \mathrm{d} x\left(W^{*} V\right)(x) \chi_{\varepsilon}(x)= & \int_{|k|>\kappa} \mathrm{d} x \mathrm{~d} k V(x) \varphi(x, k) \frac{e^{-|k| / \sqrt{\varepsilon}}}{4 \pi|k| \varepsilon} \\
& +\int_{|k| \leq \kappa} \mathrm{d} x \mathrm{~d} k V(x) \varphi(x, k) \frac{e^{-|k| / \sqrt{\varepsilon}}}{4 \pi|k| \varepsilon} .
\end{aligned}
$$

The first term on the r.h.s. of (8.48) can be controlled by

$$
\begin{aligned}
\left|\int_{|k|>\kappa} \mathrm{d} x \mathrm{~d} k V(x) \varphi(x, k) \frac{e^{-|k| / \sqrt{\varepsilon}}}{4 \pi|k| \varepsilon}\right| & =\left|\int_{|k|>\kappa} \mathrm{d} k V^{\sharp}(k) \frac{e^{-|k| / \sqrt{\varepsilon}}}{4 \pi|k| \varepsilon}\right| \\
& \leq C\left\|V^{\sharp}\right\|_{L^{2}}\left(\int_{|k| \geq \kappa} \mathrm{d} k \frac{e^{-2|k| / \sqrt{\varepsilon}}}{|k|^{2} \varepsilon^{2}}\right)^{1 / 2} \\
& \leq C\|V\|_{L^{2}} \frac{e^{-\kappa /(2 \sqrt{\varepsilon})}}{\varepsilon^{3 / 4}} \rightarrow 0,
\end{aligned}
$$

as $\varepsilon \rightarrow 0$. Here we introduced the function

$$
V^{\sharp}(k)=\text { l.i.m. }(2 \pi)^{-3 / 2} \int \mathrm{d} x V(x) \varphi(x, k),
$$

where l.i.m. denotes the $L^{2}$-limit of the integral over $|x| \leq M$ as $M \rightarrow \infty$. The existence of $V^{\sharp}$ for $V \in L^{2}\left(\mathbb{R}^{3}\right)$ and the fact that $\left\|V^{\sharp}\right\|_{L^{2}} \leq\|V\|_{L^{2}}$ (actually, in our case, $\left.\left\|V^{\sharp}\right\|_{L^{2}}=\|V\|_{L^{2}}\right)$ are proven in [24, Theorem IX.41]. As for the second term on the r.h.s. of (8.48), we have

$$
\begin{aligned}
\int_{|k| \leq \kappa} \mathrm{d} k \mathrm{~d} x V(x) \varphi(x, k) \frac{e^{-|k| / \sqrt{\varepsilon}}}{4 \pi|k| \varepsilon}= & \int_{|k| \leq \kappa} \mathrm{d} k \mathrm{~d} x V(x) \varphi(x, 0) \frac{e^{-|k| / \sqrt{\varepsilon}}}{4 \pi|k| \varepsilon} \\
& +\int_{|k| \leq \kappa} \mathrm{d} k \mathrm{~d} x V(x)(\varphi(x, k)-\varphi(x, 0)) \frac{e^{-|k| / \sqrt{\varepsilon}}}{4 \pi|k| \varepsilon} \\
= & \left(1-\left(1+\kappa \varepsilon^{-1 / 2}\right) e^{-\kappa \varepsilon^{-1 / 2}}\right) \int \mathrm{d} x V(x) \varphi(x, 0) \\
& +\int_{|k| \leq \kappa} \mathrm{d} k \mathrm{~d} x V(x)(\varphi(x, k)-\varphi(x, 0)) \frac{e^{-|k| / \sqrt{\varepsilon}}}{4 \pi|k| \varepsilon} .
\end{aligned}
$$

Using the fact that $\varphi(x, 0)$ is the solution of the zero energy scattering equation

$$
(-\Delta+(1 / 2) V(x)) \varphi(x, 0)=0
$$

with the boundary condition $\varphi(x, 0) \rightarrow 1$ as $|x| \rightarrow \infty$, it follows that (see (1.6))

$$
\int \mathrm{d} x V(x) \varphi(x, 0)=8 \pi a_{0} .
$$

To bound the second term on the r.h.s. of (8.50), we define

$$
\psi_{k}(x)=V^{1 / 2}(x) \varphi(x, k)
$$


and we observe that, from the Lippman-Schwinger equation 8.42),

$$
\begin{aligned}
\psi_{k}(x)-\psi_{0}(x)= & V^{1 / 2}(x)\left(e^{i k \cdot x}-1\right) \\
& -\frac{1}{8 \pi} \int \mathrm{d} y V^{1 / 2}(x) \frac{e^{i|k||x-y|}}{|x-y|} V^{1 / 2}(y)\left(\psi_{k}(y)-\psi_{0}(y)\right) \\
& -\frac{1}{8 \pi} \int \mathrm{d} y V^{1 / 2}(x) \frac{e^{i|k||x-y|}-1}{|x-y|} V^{1 / 2}(y) \psi_{0}(y),
\end{aligned}
$$

which implies that (with $M_{|k|}$ defined in (8.43))

$$
\begin{aligned}
& {\left[M_{|k|}\left(\psi_{k}-\psi_{0}\right)\right](x)} \\
& \quad=V^{1 / 2}(x)\left(e^{i k \cdot x}-1\right)-\frac{1}{4 \pi} \int \mathrm{d} y V^{1 / 2}(x) \frac{e^{i|k||x-y|}-1}{|x-y|} V^{1 / 2}(y) \psi_{0}(y) .
\end{aligned}
$$

By (8.46), we have

$$
\begin{aligned}
\left\|\psi_{k}-\psi_{0}\right\|_{L^{2}} & \leq C\left(\left\|V^{1 / 2}(x)\left(e^{i k \cdot x}-1\right)\right\|_{L^{2}}+\left\|V^{1 / 2}\right\|_{L^{2}}|k| \int V^{1 / 2}(y)\left|\psi_{0}(y)\right|\right) \\
& \leq C|k|\left(\left\||x|^{2} V\right\|_{L^{1}}^{1 / 2}+\|V\|_{L^{1}}^{3 / 2}\right) \\
& \leq C|k| .
\end{aligned}
$$

Therefore, the second term on the r.h.s. of (8.50) can be bounded by

$$
\begin{aligned}
& \left|\int_{|k| \leq \kappa} \mathrm{d} k \mathrm{~d} x V(x)(\varphi(x, k)-\varphi(x, 0)) \frac{e^{-|k| / \sqrt{\varepsilon}}}{4 \pi|k| \varepsilon}\right| \\
& \quad \leq \int_{|k| \leq \kappa} \mathrm{d} k \mathrm{~d} x V^{1 / 2}(x)\left|\psi_{k}(x)-\psi_{0}(x)\right| \frac{e^{-|k| / \sqrt{\varepsilon}}}{4 \pi|k| \varepsilon} \\
& \quad \leq\|V\|_{L^{1}}^{1 / 2} \int_{|k| \leq \kappa} \mathrm{d} k\left\|\psi_{k}-\psi_{0}\right\|_{L^{2}} \frac{e^{-|k| / \sqrt{\varepsilon}}}{4 \pi|k| \varepsilon} \\
& \quad \leq C\|V\|_{L^{1}}^{1 / 2} \int_{|k| \leq \kappa} \mathrm{d} k \frac{e^{-|k| / \sqrt{\varepsilon}}}{\varepsilon} \\
& \quad \leq C \varepsilon^{1 / 2},
\end{aligned}
$$

and thus it converges to zero as $\varepsilon \rightarrow 0$. The last equation, together with (8.41), (8.48), (8.49), (8.50), and (8.51), concludes the proof of the lemma.

Lemma 8.5. Suppose that $V \geq 0$ and $V(x) \leq C\langle x\rangle^{-\sigma}$, for some $\sigma \geq 5$. Then, for every $g \in L^{2}\left(\mathbb{R}^{3}, \mathrm{~d} x\right)$, we have

$$
\left\|\left(W_{N}-1\right) g\right\| \leq C N^{-1 / 6}\|g\|_{H^{1}} .
$$

Proof. Let $\mathfrak{h}_{N}=-\Delta+(1 / 2) V_{N}(x)$. Since

$$
W_{N}=s-\lim _{t \rightarrow \infty} e^{i \mathfrak{h}_{N} t} e^{i \Delta t},
$$

it is enough to prove that

$$
\sup _{t \in \mathbb{R}}\left\|\left(e^{-i \mathfrak{h}_{N} t}-e^{i \Delta t}\right) g\right\| \leq C N^{-1 / 6}\|g\|_{H^{1}} .
$$


Note that

$$
\frac{\mathrm{d}}{\mathrm{d} t}\left\|\left(e^{-i \mathfrak{h}_{N} t}-e^{i \Delta t}\right) g\right\|^{2}=2 \operatorname{Im}\left\langle e^{-i \mathfrak{h}_{N} t} g, V_{N}(x) e^{i \Delta t} g\right\rangle,
$$

which implies that

$$
\left\|\left(e^{-i \mathfrak{h}_{N} t}-e^{i \Delta t}\right) g\right\|^{2} \leq 2 \int_{0}^{t} \mathrm{~d} s\left|\left\langle e^{-i \mathfrak{h}_{N} s} g, V_{N}(x) e^{i \Delta s} g\right\rangle\right| .
$$

Next we observe that

$$
\begin{aligned}
\left|\left\langle e^{-i \mathfrak{h}_{N} s} g, V_{N}(x) e^{i \Delta s} g\right\rangle\right| & \leq\left\|e^{-i \mathfrak{h}_{N} s} g\right\|_{\infty}\left\|e^{i \Delta s} g\right\|_{\infty}\left\|V_{N}\right\|_{1} \\
& \leq \frac{\|V\|_{1}\|g\|_{1}^{2}\|W\|_{\infty \rightarrow \infty}\left\|W^{*}\right\|_{1 \rightarrow 1}}{N s^{3}},
\end{aligned}
$$

where we used the fact that

$$
\left\|W_{N}\right\|_{p \rightarrow p}=\|W\|_{p \rightarrow p}
$$

for every $N$ and $1 \leq p \leq \infty$. For small $s$ we need a different estimate of the integrand on the r.h.s. of (8.57). To this end we remark that

$$
\begin{aligned}
\left|\left\langle e^{-i \mathfrak{h}_{N} s} g, V_{N}(x) e^{i \Delta s} g\right\rangle\right| & \leq\left\langle e^{i \mathfrak{h}_{N} s} g, V_{N}(x) e^{i \mathfrak{h}_{N} s} g\right\rangle^{1 / 2}\left\langle e^{i \Delta s} g, V_{N}(x) e^{i \Delta s} g\right\rangle \\
& \leq C\left\|V_{N}\right\|_{3 / 2}\left\|\nabla e^{i \mathfrak{h}_{N} s} g\right\|\left\|\nabla e^{i \Delta s} g\right\| \\
& \leq C\|V\|_{3 / 2}\left(1+\|V\|_{3 / 2}\right)^{1 / 2}\|g\|_{H^{1}}^{2},
\end{aligned}
$$

where we used the fact that $\left\|V_{N}\right\|_{3 / 2}=\|V\|_{3 / 2}$ and we estimated

$$
\left\|\nabla e^{i \mathfrak{h}_{N} s} g\right\|^{2}=\left\langle e^{i \mathfrak{h}_{N} s} g,-\Delta e^{i \mathfrak{h}_{N} s} g\right\rangle \leq\left\langle g, \mathfrak{h}_{N} g\right\rangle \leq\left(1+\|V\|_{3 / 2}\right)\|g\|_{H^{1}}^{2} .
$$

Combining (8.58) and (8.59), we obtain from (8.57)

$$
\begin{aligned}
\left\|\left(e^{-i \mathfrak{h}_{N} t}-e^{i \Delta t}\right) g\right\|^{2} \leq & 2 \int_{0}^{N^{-\alpha}} \mathrm{d} s\|V\|_{3 / 2}\left(1+\|V\|_{3 / 2}\right)^{1 / 2}\|g\|_{H^{1}}^{2} \\
& +2 \int_{N^{-\alpha}}^{t} \mathrm{~d} s \frac{\|V\|_{1}\|g\|_{H^{1}}^{2}\|W\|_{\infty \rightarrow \infty}\left\|W^{*}\right\|_{1 \rightarrow 1}}{N s^{3}} \\
\leq & \left(C_{1} N^{-\alpha}+C_{2} N^{2 \alpha-1}\right)\|g\|_{H^{1}}^{2}
\end{aligned}
$$

for every $t \in \mathbb{R}$. Choosing $\alpha=1 / 3$, we obtain (8.56).

\section{Approximation of the initial Data}

In this section we show how to regularize the initial wave function $\psi_{N}$ given in Theorem 3.2 .

Proposition 9.1. Suppose that $\psi_{N} \in L^{2}\left(\mathbb{R}^{3 N}\right)$ with $\left\|\psi_{N}\right\|=1$ is a family of $N$-particle wave functions with

$$
\left\langle\psi_{N}, H_{N} \psi_{N}\right\rangle \leq C N
$$

and with one-particle marginal density $\gamma_{N}^{(1)}$ such that

$$
\gamma_{N}^{(1)} \rightarrow|\varphi\rangle\langle\varphi| \quad \text { as } N \rightarrow \infty
$$

for a $\varphi \in H^{1}\left(\mathbb{R}^{3}\right)$. For $\kappa>0$ we define

$$
\widetilde{\psi}_{N}:=\frac{\chi\left(\kappa H_{N} / N\right) \psi_{N}}{\left\|\chi\left(\kappa H_{N} / N\right) \psi_{N}\right\|} .
$$


Here $\chi \in C_{0}^{\infty}(\mathbb{R})$ is a cutoff function such that $0 \leq \chi \leq 1$, $\chi(s)=1$ for $0 \leq s \leq 1$ and $\chi(s)=0$ for $s \geq 2$. We denote by $\widetilde{\gamma}_{N}^{(k)}$, for $k=1, \ldots, N$, the marginal densities associated with $\widetilde{\psi}_{N}$.

i) For every integer $k \geq 1$ we have

$$
\left\langle\widetilde{\psi}_{N}, H_{N}^{k} \widetilde{\psi}_{N}\right\rangle \leq \frac{2^{k} N^{k}}{\kappa^{k}} .
$$

ii) We have

$$
\sup _{N}\left\|\psi_{N}-\widetilde{\psi}_{N}\right\| \leq C \kappa^{1 / 2}
$$

iii) For $\kappa>0$ small enough and for every fixed $k \geq 1$ we have

$$
\left.\lim _{N \rightarrow \infty} \operatorname{Tr}\left|\widetilde{\gamma}_{N}^{(k)}-\right| \varphi\right\rangle\left\langle\left.\varphi\right|^{\otimes k}\right|=0 .
$$

Proof. For the proof of part i) and ii), see [12, Proposition 8.1]. To prove iii), we begin by noticing (see (1.2)) that it is enough to show that

$$
\left.\lim _{N \rightarrow \infty} \operatorname{Tr}\left|\widetilde{\gamma}_{N}^{(1)}-\right| \varphi\right\rangle\langle\varphi||=0 .
$$

Moreover, since the limiting density is an orthogonal projection, trace-norm convergence is equivalent to weak* convergence. In other words, it is enough to prove that, for every compact operator $J^{(1)} \in \mathcal{K}_{1}$ and for every $\varepsilon>0$, there exists $N_{0}=N_{0}\left(J^{(1)}, \varepsilon\right)$ such that

$$
\left|\operatorname{Tr} J^{(1)}\left(\widetilde{\gamma}_{N}^{(1)}-|\varphi\rangle\langle\varphi|\right)\right| \leq \varepsilon
$$

for $N>N_{0}$. To show (9.6), we start by observing that, from (9.2), there exists a sequence $\xi_{N}^{(N-1)} \in L^{2}\left(\mathbb{R}^{3(N-1)}\right)$ with $\left\|\xi_{N}^{(N-1)}\right\|=1$ such that

$$
\left\|\psi_{N}-\varphi \otimes \xi_{N}^{(N-1)}\right\| \rightarrow 0 \quad \text { as } N \rightarrow \infty .
$$

This was proven by Alessandro Michelangeli in 23] using the following argument. Choose an orthonormal basis $\left\{f_{i}\right\}_{i \geq 1}$ of $L^{2}\left(\mathbb{R}^{3}\right)$ with $f_{1}=\varphi$. Also choose an orthonormal basis $\left\{g_{j}\right\}_{j \geq 1}$ of $L^{2}\left(\mathbb{R}^{3(N-1)}\right)$. Then one can write

$$
\psi_{N}=\sum_{i j} \alpha_{i j}^{(N)} f_{i} \otimes g_{j}
$$

and

$$
\left|\psi_{N}\right\rangle\left\langle\psi_{N}\left|=\sum_{i, j, i^{\prime}, j^{\prime}} \bar{\alpha}_{i, j}^{(N)} \alpha_{i^{\prime}, j^{\prime}}^{(N)}\right| f_{i}\right\rangle\left\langle f_{i^{\prime}}|\otimes| g_{j}\right\rangle\left\langle g_{j^{\prime}}\right| .
$$

This implies that

$$
\begin{aligned}
\gamma_{N}^{(1)}=\sum_{j}\left(\left|\alpha_{1, j}^{(N)}\right|^{2}|\varphi\rangle\langle\varphi|\right. & +\alpha_{1, j}^{(N)} \sum_{i \neq 1} \bar{\alpha}_{i, j}^{(N)}|\varphi\rangle\left\langle f_{i}\right| \\
& \left.+\bar{\alpha}_{1, j}^{(N)} \sum_{i \neq 1} \alpha_{i, j}^{(N)}\left|f_{i}\right\rangle\left\langle\varphi\left|+\sum_{i, i^{\prime} \neq 1} \bar{\alpha}_{i, j}^{(N)} \alpha_{i^{\prime}, j}^{(N)}\right| f_{i}\right\rangle\left\langle f_{i^{\prime}}\right|\right)
\end{aligned}
$$

and therefore, using (9.2), that

$$
\sum_{j}\left|\alpha_{1, j}^{(N)}\right|^{2} \rightarrow 1
$$


as $N \rightarrow \infty$. Thus, putting $\widetilde{\xi}_{N}^{(N-1)}=\sum_{j} \alpha_{1, j}^{(N)} g_{j}$, we get

$$
\left\|\psi_{N}-\varphi \otimes \widetilde{\xi}_{N}^{(N-1)}\right\|^{2}=\sum_{j} \sum_{i \neq 1}\left|\alpha_{i, j}^{(N)}\right|^{2}=1-\sum_{j}\left|\alpha_{1, j}^{(N)}\right|^{2} \rightarrow 0
$$

as $N \rightarrow \infty$. It is then simple to check that $\xi_{N}^{(N-1)}=\widetilde{\xi}_{N}^{(N-1)} /\left\|\widetilde{\xi}_{N}^{(N-1)}\right\|$ satisfies (9.7).

On the other hand, there exists $\varphi_{*} \in H^{2}\left(\mathbb{R}^{3}\right)$ with $\left\|\varphi_{*}\right\|=1$ and such that

$$
\left\|\varphi-\varphi_{*}\right\| \leq \frac{\varepsilon}{32\left\|J^{(1)}\right\|} .
$$

Let $\Xi=\chi\left(\kappa H_{N} / N\right)$. Then

$$
\left\|(\Xi-1) \psi_{N}\right\|^{2} \leq \frac{\kappa}{N}\left\langle\psi_{N}, H_{N} \psi_{N}\right\rangle \leq C \kappa
$$

independently of $N$. Therefore, choosing $\kappa>0$ so small that $\left\|\Xi \psi_{N}\right\| \geq 1 / 2$, we find

$$
\begin{aligned}
& \left\|\frac{\Xi \psi_{N}}{\left\|\Xi \psi_{N}\right\|}-\frac{\Xi\left(\varphi_{*} \otimes \xi_{N}^{(N-1)}\right)}{\left\|\Xi\left(\varphi_{*} \otimes \xi_{N}^{(N-1)}\right)\right\|}\right\| \leq \frac{2}{\left\|\Xi \psi_{N}\right\|}\left\|\Xi\left(\psi_{N}-\varphi_{*} \otimes \xi_{N}^{(N-1)}\right)\right\| \\
& \leq 4\left\|\psi_{N}-\varphi_{*} \otimes \xi_{N}^{(N-1)}\right\| \\
& \leq 4\left\|\psi_{N}-\varphi \otimes \xi_{N}^{(N-1)}\right\|+4\left\|\varphi-\varphi_{*}\right\| \\
& \leq \frac{\varepsilon}{6\left\|J^{(1)}\right\|}
\end{aligned}
$$

for all $N$ sufficiently large. Next we define the Hamiltonian

$$
\widehat{H}_{N}:=-\sum_{j=2}^{N} \Delta_{j}+\sum_{1<i<j}^{N} V_{N}\left(x_{i}-x_{j}\right) .
$$

Note that $\widehat{H}_{N}$ acts only on the last $N-1$ variables. We set $\widehat{\Xi}:=\chi\left(\kappa \widehat{H}_{N} / N\right)$. Then we claim that, if $\varepsilon>0$ is small enough,

$$
\left\|\frac{\Xi \psi_{N}}{\left\|\Xi \psi_{N}\right\|}-\frac{\widehat{\Xi}\left(\varphi_{*} \otimes \xi_{N}^{(N-1)}\right)}{\left\|\widehat{\Xi}\left(\varphi_{*} \otimes \xi_{N}^{(N-1)}\right)\right\|}\right\| \leq \frac{\varepsilon}{3\left\|J^{(1)}\right\|}
$$

for $N$ sufficiently large. The proof of (9.10) can be found in [12, Proposition 8.1]. To get (9.6) we define

$$
\widehat{\psi}_{N}:=\frac{\widehat{\Xi}\left(\varphi_{*} \otimes \xi_{N}^{(N-1)}\right)}{\left\|\widehat{\Xi}\left(\varphi_{*} \otimes \xi_{N}^{(N-1)}\right)\right\|}=\varphi_{*} \otimes \frac{\widehat{\Xi} \xi_{N}^{(N-1)}}{\left\|\widehat{\Xi} \xi_{N}^{(N-1)}\right\|},
$$

where we used the fact that $\widehat{\Xi}$ acts only on the last $N-1$ variables and the fact that $\left\|\varphi_{*}\right\|=1$. Define

$$
\widehat{\gamma}_{N}^{(1)}\left(x_{1} ; x_{1}^{\prime}\right):=\int \mathrm{d} \mathbf{x}_{N-1} \widehat{\psi}_{N}\left(x_{1}, \mathbf{x}_{N-k}\right) \overline{\widehat{\psi}}_{N}\left(x_{1}^{\prime}, \mathbf{x}_{N-k}\right) .
$$

Note that $\widehat{\psi}_{N}$ is not symmetric in all variables, but it is symmetric in the last $N-1$ variables. In particular, $\widehat{\gamma}_{N}^{(1)}$ is a density matrix and clearly $\widehat{\gamma}_{N}^{(1)}=\left|\varphi_{*}\right\rangle\left\langle\varphi_{*}\right|$. Therefore, since $\left\|\widetilde{\psi}_{N}-\widehat{\psi}_{N}\right\| \leq \varepsilon /\left(3\left\|J^{(1)}\right\|\right)$ by (9.10) and since $\left\|\varphi-\varphi_{*}\right\| \leq \varepsilon /\left(32\left\|J^{(1)}\right\|\right)$, 
we have

$$
\begin{aligned}
\left|\operatorname{Tr} J^{(1)}\left(\widetilde{\gamma}_{N}^{(1)}-|\varphi\rangle\langle\varphi|\right)\right| \leq & \left|\operatorname{Tr} J^{(1)}\left(\widetilde{\gamma}_{N}^{(1)}-\left|\varphi_{*}\right\rangle\left\langle\varphi_{*}\right|\right)\right| \\
& +\left|\operatorname{Tr} J^{(1)}\left(\left|\varphi_{*}\right\rangle\left\langle\varphi_{*}|-| \varphi\right\rangle\langle\varphi|\right)\right| \\
\leq & 2\left\|J^{(1)}\right\|\left\|\widetilde{\psi}_{N}-\widehat{\psi}_{N}\right\|+2\left\|J^{(1)}\right\|\left\|\varphi-\varphi_{*}\right\| \leq \varepsilon
\end{aligned}
$$

for $N$ sufficiently large (for arbitrary $\kappa, \varepsilon>0$ small enough). This proves (9.6).

\section{Poincaré-Sobolev type inequalities}

In the proof of the convergence we need to estimate potentials converging to a delta function, and their difference to a normalized $\delta$-function. To this end we make use of the following three lemmas.

Lemma 10.1. Suppose $V \in L^{1}\left(\mathbb{R}^{3}\right)$. Then

$$
\begin{aligned}
\left|\left\langle\varphi, V\left(x_{1}-x_{2}\right) \psi\right\rangle\right| \leq C\|V\|_{1}\langle\psi & \left.,\left(\left(\nabla_{1} \cdot \nabla_{2}\right)^{2}-\Delta_{1}-\Delta_{2}+1\right) \psi\right\rangle^{1 / 2} \\
& \times\left\langle\varphi,\left(\left(\nabla_{1} \cdot \nabla_{2}\right)^{2}-\Delta_{1}-\Delta_{2}+1\right) \varphi\right\rangle^{1 / 2}
\end{aligned}
$$

for every $\psi, \varphi \in L^{2}\left(\mathbb{R}^{6}, \mathrm{~d} x_{1}, \mathrm{~d} x_{2}\right)$.

Proof. Switching to Fourier space, we find

$$
\begin{aligned}
\left\langle\varphi, V\left(x_{1}-x_{2}\right) \psi\right\rangle=\int \mathrm{d} p_{1} \mathrm{~d} p_{2} \mathrm{~d} q_{1} \mathrm{~d} q_{2} \overline{\widehat{\varphi}\left(p_{1}, p_{2}\right)} \widehat{\psi}\left(q_{1}, q_{2}\right) \\
\times \widehat{V}\left(q_{1}-p_{1}\right) \delta\left(p_{1}+p_{2}-q_{1}-q_{2}\right) .
\end{aligned}
$$

Therefore, by a weighted Schwarz inequality,

$$
\begin{aligned}
& \left|\left\langle\varphi, V\left(x_{1}-x_{2}\right) \psi\right\rangle\right| \\
& \leq\|\widehat{V}\|_{\infty}\left(\int \mathrm{d} p_{1} \mathrm{~d} p_{2} \mathrm{~d} q_{1} \mathrm{~d} q_{2} \frac{\left(p_{1} \cdot p_{2}\right)^{2}+p_{1}^{2}+p_{2}^{2}+1}{\left(q_{1} \cdot q_{2}\right)^{2}+q_{1}^{2}+q_{2}^{2}+1}\right. \\
& \left.\quad \times\left|\widehat{\varphi}\left(p_{1}, p_{2}\right)\right|^{2} \delta\left(p_{1}+p_{2}-q_{1}-q_{2}\right)\right)^{1 / 2} \\
& \quad \times\left(\int \mathrm{d} p_{1} \mathrm{~d} p_{2} \mathrm{~d} q_{1} \mathrm{~d} q_{2} \frac{\left(q_{1} \cdot q_{2}\right)^{2}+q_{1}^{2}+q_{2}^{2}+1}{\left(p_{1} \cdot p_{2}\right)^{2}+p_{1}^{2}+p_{2}^{2}+1}\left|\widehat{\psi}\left(q_{1}, q_{2}\right)\right|^{2} \delta\left(p_{1}+p_{2}-q_{1}-q_{2}\right)\right)^{1 / 2} \\
& \leq\|V\|_{1}\left(\sup _{p} \int \mathrm{d} q \frac{1}{(q \cdot(p-q))^{2}+q^{2}+(p-q)^{2}+1}\right) \\
& \quad \times\left\langle\psi,\left(\left(\nabla_{1} \cdot \nabla_{2}\right)^{2}-\Delta_{1}-\Delta_{2}+1\right) \psi\right\rangle^{1 / 2}\left\langle\varphi,\left(\left(\nabla_{1} \cdot \nabla_{2}\right)^{2}-\Delta_{1}-\Delta_{2}+1\right) \varphi\right\rangle^{1 / 2} .
\end{aligned}
$$

The lemma will then follow from

$$
\sup _{p \in \mathbb{R}^{3}} \int \mathrm{d} q \frac{1}{(q \cdot(p-q))^{2}+q^{2}+(p-q)^{2}+1}<\infty .
$$


To prove (10.4), we proceed as follows:

$$
\begin{aligned}
\int \mathrm{d} q & \frac{1}{(q \cdot(p-q))^{2}+q^{2}+(p-q)^{2}+1} \\
= & \int_{\left|q-\frac{p}{2}\right|>|p|} \mathrm{d} q \frac{1}{\left(\left(q-\frac{p}{2}\right)^{2}-\frac{p^{2}}{4}\right)^{2}+q^{2}+(p-q)^{2}+1} \\
& +\int_{\left|q-\frac{p}{2}\right|<|p|} \mathrm{d} q \frac{1}{\left(\left(q-\frac{p}{2}\right)^{2}-\frac{p^{2}}{4}\right)^{2}+q^{2}+(p-q)^{2}+1} .
\end{aligned}
$$

The first term on the r.h.s. of the last equation is bounded by

$$
\begin{aligned}
\int_{\left|q-\frac{p}{2}\right|>|p|} \mathrm{d} q \frac{1}{\left(\left(q-\frac{p}{2}\right)^{2}-\frac{p^{2}}{4}\right)^{2}+q^{2}+(p-q)^{2}+1} & \leq \int_{\left|q-\frac{p}{2}\right|>|p|} \mathrm{d} q \frac{1}{\frac{9}{16}\left|q-\frac{p}{2}\right|^{4}+1} \\
& \leq \frac{16}{9} \int_{\mathbb{R}^{3}} \mathrm{~d} q \frac{1}{|q|^{4}+1}<\infty,
\end{aligned}
$$

uniformly in $p \in \mathbb{R}^{3}$. As for the second term on the r.h.s. of (10.5), we observe that

$$
\begin{aligned}
\int_{\left|q-\frac{p}{2}\right|<|p|} \mathrm{d} q & \frac{1}{\left(\left(q-\frac{p}{2}\right)^{2}-\frac{p^{2}}{4}\right)^{2}+q^{2}+(p-q)^{2}+1} \\
& =\int_{|x|<|p|} \mathrm{d} x \frac{1}{\left(x^{2}-\frac{p^{2}}{4}\right)^{2}+\left(x+\frac{p}{2}\right)^{2}+\left(x-\frac{p}{2}\right)^{2}+1} \\
& =4 \pi \int_{0}^{|p|} \mathrm{d} r \frac{r^{2}}{\left(r^{2}-\frac{|p|^{2}}{4}\right)^{2}+2 r^{2}+\frac{|p|^{2}}{2}+1} \\
& \leq C|p|^{2} \int_{-|p| / 2}^{|p| / 2} \mathrm{~d} r \frac{1}{r^{2}(r+|p|)^{2}+\left(r+\frac{|p|}{2}\right)^{2}+\frac{|p|^{2}}{4}+1} \\
& \leq C \int_{-|p| / 2}^{|p| / 2} \mathrm{~d} r \frac{1}{r^{2}+1} \leq C \int_{\mathbb{R}} \mathrm{d} r \frac{1}{r^{2}+1}<\infty,
\end{aligned}
$$

uniformly in $p$.

Lemma 10.2. Suppose $V \in L^{1}\left(\mathbb{R}^{3}\right)$ with $\int V(x) \mathrm{d} x=1$. For $\alpha>0$, let $V_{\alpha}(x)=$ $\alpha^{-3} V(x / \alpha)$. Then there exists a sequence $\beta_{\alpha}$ with $\beta_{\alpha} \rightarrow 0$ as $\alpha \rightarrow 0$ such that

$$
\begin{aligned}
& \quad\left|\left\langle\varphi,\left(V_{\alpha}\left(x_{1}-x_{2}\right)-\delta\left(x_{1}-x_{2}\right)\right) \psi\right\rangle\right| \\
& \leq C \beta_{\alpha}\left\langle\psi,\left(\left(\nabla_{1} \cdot \nabla_{2}\right)^{2}-\Delta_{1}-\Delta_{2}+1\right) \psi\right\rangle^{1 / 2} \\
& \quad \times\left\langle\varphi,\left(\left(\nabla_{1}-\nabla_{2}\right)^{4}+\left(\nabla_{1}+\nabla_{2}\right)^{2}+1\right) \varphi\right\rangle^{1 / 2},
\end{aligned}
$$

for all $\varphi, \psi \in L^{2}\left(\mathbb{R}^{6}\right)$.

Proof. Switching to Fourier space we find

$$
\left\langle\varphi,\left(V_{\alpha}\left(x_{1}-x_{2}\right)-\delta\left(x_{1}-x_{2}\right)\right) \psi\right\rangle=\mathrm{I}+\mathrm{II}
$$


where we defined

$$
\begin{aligned}
\mathrm{I}=\int_{\left|x \cdot\left(p_{1}-q_{1}\right)\right|<\alpha^{-1 / 2}} \mathrm{~d} p_{1} \mathrm{~d} p_{2} \mathrm{~d} q_{1} \mathrm{~d} q_{2} \mathrm{~d} x V(x) \overline{\widehat{\varphi}}\left(p_{1}, p_{2}\right) \\
\times\left(e^{i \alpha x \cdot\left(p_{1}-q_{1}\right)}-1\right) \widehat{\psi}\left(q_{1}, q_{2}\right) \delta\left(p_{1}+p_{2}-q_{1}-q_{2}\right), \\
\mathrm{II}=\int_{\left|x \cdot\left(p_{1}-q_{1}\right)\right| \geq \alpha^{-1 / 2}} \mathrm{~d} p_{1} \mathrm{~d} p_{2} \mathrm{~d} q_{1} \mathrm{~d} q_{2} \mathrm{~d} x V(x) \overline{\hat{\varphi}}\left(p_{1}, p_{2}\right) \\
\times\left(e^{i \alpha x \cdot\left(p_{1}-q_{1}\right)}-1\right) \widehat{\psi}\left(q_{1}, q_{2}\right) \delta\left(p_{1}+p_{2}-q_{1}-q_{2}\right) .
\end{aligned}
$$

To bound the first term we use the fact that $\left|e^{i \kappa}-1\right| \leq|\kappa|$, for $\kappa \in \mathbb{R}$, and we observe that

$$
\begin{aligned}
|\mathrm{I}| \leq & \alpha^{1 / 2}\|V\|_{1} \int \mathrm{d} p_{1} \mathrm{~d} p_{2} \mathrm{~d} q_{1} \mathrm{~d} q_{2} \frac{\sqrt{\left|p_{1}-p_{2}\right|^{4}+\left(p_{1}+p_{2}\right)^{2}+1}}{\sqrt{\left(q_{1} \cdot q_{2}\right)^{2}+q_{1}^{2}+q_{2}^{2}+1}}\left|\widehat{\varphi}\left(p_{1}, p_{2}\right)\right| \\
& \times \frac{\sqrt{\left(q_{1} \cdot q_{2}\right)^{2}+q_{1}^{2}+q_{2}^{2}+1}}{\sqrt{\left|p_{1}-p_{2}\right|^{4}+\left(p_{1}+p_{2}\right)^{2}+1}}\left|\widehat{\psi}\left(q_{1}, q_{2}\right)\right| \delta\left(p_{1}+p_{2}-q_{1}-q_{2}\right) .
\end{aligned}
$$

With a Schwarz inequality, we obtain that

$$
\begin{aligned}
|\mathrm{I}| \leq & \alpha^{1 / 2}\|V\|_{1}\left(\int \mathrm{d} p_{1} \mathrm{~d} p_{2} \mathrm{~d} q_{1} \mathrm{~d} q_{2} \frac{\left|p_{1}-p_{2}\right|^{4}+\left(p_{1}+p_{2}\right)^{2}+1}{\left(q_{1} \cdot q_{2}\right)^{2}+q_{1}^{2}+q_{2}^{2}+1}\right. \\
& \left.\times\left|\widehat{\varphi}\left(p_{1}, p_{2}\right)\right|^{2} \delta\left(p_{1}+p_{2}-q_{1}-q_{2}\right)\right)^{1 / 2} \\
& \times\left(\int \mathrm{d} p_{1} \mathrm{~d} p_{2} \mathrm{~d} q_{1} \mathrm{~d} q_{2} \frac{\sqrt{\left(q_{1} \cdot q_{2}\right)^{2}+q_{1}^{2}+q_{2}^{2}+1}}{\sqrt{\left|p_{1}-p_{2}\right|^{4}+\left(p_{1}+p_{2}\right)^{2}+1}}\right. \\
& \times \mid \widehat{\psi}\left(q_{1},\left.q_{2}\right|^{2} \delta\left(p_{1}+p_{2}-q_{1}-q_{2}\right)\right)^{1 / 2} \\
\leq & \alpha^{1 / 2}\|V\|_{1}\left\langle\varphi,\left(\left(\nabla_{1}-\nabla_{2}\right)^{4}+\left(\nabla_{1}+\nabla_{2}\right)^{2}+1\right) \varphi\right\rangle^{1 / 2} \\
& \times\left\langle\psi,\left(\left(\nabla_{1} \cdot \nabla_{2}\right)^{2}-\Delta_{1}-\Delta_{2}+1\right) \psi\right\rangle^{1 / 2} \\
& \times\left(\sup _{p \in \mathbb{R}^{3}} \int \frac{\mathrm{d} q}{|q-p|^{4}+p^{2}+1}\right)^{1 / 2} . \\
& \times\left(\sup _{q \in \mathbb{R}^{3}} \int \frac{\mathrm{d} p}{(p \cdot(q-p))^{2}+p^{2}+(q-p)^{2}+1}\right)^{1 / 2} .
\end{aligned}
$$

From

$$
\sup _{p \in \mathbb{R}^{3}} \int \frac{\mathrm{d} q}{|q-p|^{4}+p^{2}+1} \leq \int \frac{\mathrm{d} q}{|q|^{4}+1}<\infty
$$

and (10.4) it follows that

$$
\begin{aligned}
|\mathrm{I}| \leq & C \alpha^{1 / 2}\left\langle\varphi,\left(\left(\nabla_{1}-\nabla_{2}\right)^{4}+\left(\nabla_{1}+\nabla_{2}\right)^{2}+1\right) \varphi\right\rangle^{1 / 2} \\
& \times\left\langle\psi,\left(\left(\nabla_{1} \cdot \nabla_{2}\right)^{2}-\Delta_{1}-\Delta_{2}+1\right) \psi\right\rangle^{1 / 2}
\end{aligned}
$$


In order to control the second term in (10.9), we bound it by

$$
\begin{aligned}
& |\mathrm{II}| \leq 2 \int_{\left|x \cdot\left(p_{1}-q_{1}\right)\right| \geq \alpha^{-1 / 2}} \mathrm{~d} p_{1} \mathrm{~d} p_{2} \mathrm{~d} q_{1} \mathrm{~d} q_{2} \mathrm{~d} x|V(x)|\left|\widehat{\varphi}\left(p_{1}, p_{2}\right)\right| \\
& \times\left|\widehat{\psi}\left(q_{1}, q_{2}\right)\right| \delta\left(p_{1}+p_{2}-q_{1}-q_{2}\right) \\
& \leq 2 \int_{|x| \geq \alpha^{-1 / 4}} \mathrm{~d} p_{1} \mathrm{~d} p_{2} \mathrm{~d} q_{1} \mathrm{~d} q_{2} \mathrm{~d} x|V(x)|\left|\widehat{\varphi}\left(p_{1}, p_{2}\right)\right| \\
& \times\left|\widehat{\psi}\left(q_{1}, q_{2}\right)\right| \delta\left(p_{1}+p_{2}-q_{1}-q_{2}\right) \\
& +2 \int_{\left|p_{1}-q_{1}\right| \geq \alpha^{-1 / 4}} \mathrm{~d} p_{1} \mathrm{~d} p_{2} \mathrm{~d} q_{1} \mathrm{~d} q_{2} \mathrm{~d} x|V(x)|\left|\varphi\left(p_{1}, p_{2}\right)\right| \\
& \times\left|\psi\left(q_{1}, q_{2}\right)\right| \delta\left(p_{1}+p_{2}-q_{1}-q_{2}\right) \\
& \leq \beta_{1, \alpha} \int \mathrm{d} p_{1} \mathrm{~d} p_{2} \mathrm{~d} q_{1} \mathrm{~d} q_{2}\left|\widehat{\varphi}\left(p_{1}, p_{2}\right)\right|\left|\widehat{\psi}\left(q_{1}, q_{2}\right)\right| \delta\left(p_{1}+p_{2}-q_{1}-q_{2}\right) \\
& +2\|V\|_{1} \int_{\left|p_{1}-q_{1}\right| \geq \alpha^{-1 / 4}} \mathrm{~d} p_{1} \mathrm{~d} p_{2} \mathrm{~d} q_{1} \mathrm{~d} q_{2}\left|\widehat{\varphi}\left(p_{1}, p_{2}\right)\right| \\
& \times\left|\widehat{\psi}\left(q_{1}, q_{2}\right)\right| \delta\left(p_{1}+p_{2}-q_{1}-q_{2}\right) \\
& \leq \beta_{1, \alpha}\left\langle\varphi,\left(\left(\nabla_{1}-\nabla_{2}\right)^{4}+\left(\nabla_{1}+\nabla_{2}\right)^{2}+1\right) \varphi\right\rangle^{1 / 2} \\
& \times\left\langle\psi,\left(\left(\nabla_{1} \cdot \nabla_{2}\right)^{4}-\Delta_{1}-\Delta_{2}+1\right) \psi\right\rangle^{1 / 2} \\
& +2\|V\|_{1} \int_{\left|p_{1}-q_{1}\right| \geq \alpha^{-1 / 4}} \mathrm{~d} p_{1} \mathrm{~d} p_{2} \mathrm{~d} q_{1} \mathrm{~d} q_{2}\left|\widehat{\varphi}\left(p_{1}, p_{2}\right)\right|\left|\widehat{\psi}\left(q_{1}, q_{2}\right)\right| \delta\left(p_{1}+p_{2}-q_{1}-q_{2}\right),
\end{aligned}
$$

where we defined

$$
\beta_{1, \alpha}=2 \int_{|x| \geq \alpha^{-1 / 4}}|V(x)|
$$

and we bounded the first integral analogously as we did with the integral in (10.10). Note that $\beta_{1, \alpha} \rightarrow 0$ as $\alpha \rightarrow 0$, because $V \in L^{1}\left(\mathbb{R}^{3}\right)$. We still need to control the last integral on the r.h.s. of the last equation. To this end, we observe that

$$
\begin{aligned}
& \int_{\left|p_{1}-q_{1}\right| \geq \alpha^{-1 / 4}} \mathrm{~d} p_{1} \mathrm{~d} p_{2} \mathrm{~d} q_{1} \mathrm{~d} q_{2}\left|\widehat{\varphi}\left(p_{1}, p_{2}\right)\right|\left|\widehat{\psi}\left(q_{1}, q_{2}\right)\right| \delta\left(p_{1}+p_{2}-q_{1}-q_{2}\right) \\
& \leq 2 \int_{\left|q_{1}\right| \geq \alpha^{-1 / 4} / 8} \mathrm{~d} p_{1} \mathrm{~d} p_{2} \mathrm{~d} q_{1} \mathrm{~d} q_{2}\left|\widehat{\varphi}\left(p_{1}, p_{2}\right)\right|\left|\widehat{\psi}\left(q_{1}, q_{2}\right)\right| \delta\left(p_{1}+p_{2}-q_{1}-q_{2}\right) \\
&+\int_{\left|q_{2}\right| \geq \alpha^{-1 / 4} / 8} \mathrm{~d} p_{1} \mathrm{~d} p_{2} \mathrm{~d} q_{1} \mathrm{~d} q_{2}\left|\widehat{\varphi}\left(p_{1}, p_{2}\right)\right|\left|\widehat{\psi}\left(q_{1}, q_{2}\right)\right| \delta\left(p_{1}+p_{2}-q_{1}-q_{2}\right) \\
&+\int_{\substack{\left|p_{1}\right| \geq \alpha^{-1 / 4} / 2 \\
\left|q_{1}+q_{2}\right| \leq \alpha^{-1 / 4} / 4}} \mathrm{~d} p_{1} \mathrm{~d} p_{2} \mathrm{~d} q_{1} \mathrm{~d} q_{2}\left|\widehat{\varphi}\left(p_{1}, p_{2}\right)\right| \widehat{\psi}\left(q_{1}, q_{2}\right) \mid \delta\left(p_{1}+p_{2}-q_{1}-q_{2}\right) .
\end{aligned}
$$


The first two terms can be bounded by

$$
\begin{gathered}
\int_{\left|q_{j}\right| \geq \alpha^{-1 / 4} / 8} \mathrm{~d} p_{1} \mathrm{~d} p_{2} \mathrm{~d} q_{1} \mathrm{~d} q_{2}\left|\widehat{\varphi}\left(p_{1}, p_{2}\right)\right|\left|\widehat{\psi}\left(q_{1}, q_{2}\right)\right| \delta\left(p_{1}+p_{2}-q_{1}-q_{2}\right) \\
\leq C \alpha^{1 / 12}\left\langle\varphi,\left(\left(\nabla_{1}-\nabla_{2}\right)^{4}+\left(\nabla_{1}+\nabla_{2}\right)^{2}+1\right) \varphi\right\rangle^{1 / 2} \\
\times\left\langle\psi,\left(\left(\nabla_{1} \cdot \nabla_{2}\right)^{4}-\Delta_{1}-\Delta_{2}+1\right) \psi\right\rangle^{1 / 2}
\end{gathered}
$$

which holds for both $j=1,2$ and for a universal constant $C$, independent of $\alpha, \varphi, \psi$. To show (10.14) note that, proceeding as in (10.10) (for example for $j=1$ ), we have

$$
\begin{aligned}
& \int_{\left|q_{1}\right| \geq \alpha^{-1 / 4} / 8} \mathrm{~d} p_{1} \mathrm{~d} p_{2} \mathrm{~d} q_{1} \mathrm{~d} q_{2}\left|\widehat{\varphi}\left(p_{1}, p_{2}\right)\right|\left|\widehat{\psi}\left(q_{1}, q_{2}\right)\right| \delta\left(p_{1}+p_{2}-q_{1}-q_{2}\right) \\
& \leq\left\langle\varphi,\left(\left(\nabla_{1}-\nabla_{2}\right)^{4}+\left(\nabla_{1}+\nabla_{2}\right)^{2}+1\right) \varphi\right\rangle^{1 / 2}\left\langle\psi,\left(\left(\nabla_{1} \cdot \nabla_{2}\right)^{4}-\Delta_{1}-\Delta_{2}+1\right) \psi\right\rangle^{1 / 2} \\
& \quad \times\left(\sup _{q \in \mathbb{R}^{3}} \int \frac{\mathrm{d} p}{(p \cdot(q-p))^{2}+p^{2}+(q-p)^{2}+1}\right)^{1 / 2} \\
& \quad \times\left(\sup _{p \in \mathbb{R}^{3}} \int_{|q| \geq \alpha^{-1 / 4} / 8} \frac{\mathrm{d} q}{|q-p|^{4}+p^{2}+1}\right)^{1 / 2},
\end{aligned}
$$

and thus (10.14) follows from (10.4) and

$$
\begin{aligned}
\sup _{p \in \mathbb{R}^{3}} & \int_{|q| \geq \alpha^{-1 / 4} / 8} \frac{\mathrm{d} q}{|q-p|^{4}+p^{2}+1} \\
& \leq\left(8 \alpha^{1 / 4}\right)^{1 / 3} \sup _{p \in \mathbb{R}^{3}} \int \mathrm{d} q \frac{|q|^{1 / 3}}{|q-p|^{4}+p^{2}+1} \\
& \leq\left(8 \alpha^{1 / 4}\right)^{1 / 3}\left(\sup _{q, p \in \mathbb{R}^{3}} \frac{|q+p|^{1 / 3}}{\left(|q|^{4}+p^{2}+1\right)^{1 / 6}}\right) \int \frac{\mathrm{d} q}{\left(|q|^{4}+1\right)^{5 / 6}} \\
& \leq C \alpha^{1 / 12} .
\end{aligned}
$$

As for the last term on the r.h.s. of (10.13), we note that

$$
\begin{aligned}
& \int_{\substack{\left|p_{1}\right| \geq q_{2}\left|\leq \alpha^{-1 / 4} / 4\\
\right| p_{1} / 2}} \mathrm{~d} p_{1} \mathrm{~d} p_{2} \mathrm{~d} q_{1} \mathrm{~d} q_{2}\left|\widehat{\varphi}\left(p_{1}, p_{2}\right)\right|\left|\widehat{\psi}\left(q_{1}, q_{2}\right)\right| \delta\left(p_{1}+p_{2}-q_{1}-q_{2}\right) \\
& \leq\left\langle\varphi,\left(\left(\nabla_{1}-\nabla_{2}\right)^{4}+\left(\nabla_{1}+\nabla_{2}\right)^{2}+1\right) \varphi\right\rangle^{1 / 2}\left\langle\psi,\left(\left(\nabla_{1} \cdot \nabla_{2}\right)^{4}-\Delta_{1}-\Delta_{2}+1\right) \psi\right\rangle^{1 / 2} \\
& \times\left(\sup _{p \in \mathbb{R}^{3}} \int \frac{\mathrm{d} q}{|q-p|^{4}+p^{2}+1}\right)^{1 / 2} \\
& \times\left(\sup _{q} \int_{|p| \geq \alpha^{-1 / 4} / 2} \frac{\mathrm{d} p}{(p \cdot(q-p))^{2}+p^{2}+(q-p)^{2}+1}\right)^{1 / 2} .
\end{aligned}
$$


Since

$$
\begin{aligned}
\int_{|p| \geq \alpha^{-1 / 4}|2| 2 \mid} & \frac{\mathrm{d} p}{(p \cdot(q-p))^{2}+p^{2}+(q-p)^{2}+1} \\
& \leq\left(2 \alpha^{1 / 4}\right)^{1 / 3} \int_{|p| \geq 2|q|} \mathrm{d} p \frac{|p|^{1 / 3}}{\left(\left(p-\frac{q}{2}\right)^{2}-\frac{q^{2}}{4}\right)^{2}+p^{2}+(q-p)^{2}+1} \\
& \leq C \alpha^{1 / 12} \int_{|p| \geq|q|} \mathrm{d} p \frac{\left|p+\frac{q}{2}\right|^{1 / 2}}{\left(p^{2}-\frac{q^{2}}{4}\right)^{2}+p^{2}+\frac{q^{2}}{4}+1} \\
& \leq C \alpha^{1 / 12} \int \mathrm{d} p \frac{|p|^{1 / 2}}{\frac{9}{16}|p|^{4}+1} \\
& \leq C \alpha^{1 / 12},
\end{aligned}
$$

it follows that the last term on the r.h.s. of (10.13) is bounded by

$$
\begin{aligned}
& \int_{\substack{\left|p_{1}\right| \geq \alpha^{-1 / 4 / 2} \\
\left|q_{1}+q_{2}\right| \leq \alpha^{-1 / 4} / 2}} \mathrm{~d} p_{1} \mathrm{~d} p_{2} \mathrm{~d} q_{1} \mathrm{~d} q_{2}\left|\widehat{\varphi}\left(p_{1}, p_{2}\right)\right|\left|\widehat{\psi}\left(q_{1}, q_{2}\right)\right| \delta\left(p_{1}+p_{2}-q_{1}-q_{2}\right) \\
& \leq C \alpha^{1 / 12}\left\langle\varphi,\left(\left(\nabla_{1}-\nabla_{2}\right)^{4}+\left(\nabla_{1}+\nabla_{2}\right)^{2}+1\right) \varphi\right\rangle^{1 / 2} \\
& \quad \times\left\langle\psi,\left(\left(\nabla_{1} \cdot \nabla_{2}\right)^{4}-\Delta_{1}-\Delta_{2}+1\right) \psi\right\rangle^{1 / 2} .
\end{aligned}
$$

From the last equation, (10.14), (10.13), and (10.12), it follows that

$$
\begin{aligned}
|\mathrm{II}| \leq C & \left(\beta_{1, \alpha}+\alpha^{1 / 12}\right) \\
& \times\left\langle\varphi,\left(\left(\nabla_{1}-\nabla_{2}\right)^{4}+\left(\nabla_{1}+\nabla_{2}\right)^{2}+1\right) \varphi\right\rangle^{1 / 2} \\
& \times\left\langle\psi,\left(\left(\nabla_{1} \cdot \nabla_{2}\right)^{4}-\Delta_{1}-\Delta_{2}+1\right) \psi\right\rangle^{1 / 2} .
\end{aligned}
$$

This together with (10.11), implies (10.8) with $\beta_{\alpha}=C\left(\beta_{1, \alpha}+\alpha^{1 / 12}+\alpha^{1 / 2}\right)$.

When dealing with the limit points $\gamma_{\infty, t}^{(k)}$, for which we have stronger a priori estimates, we will make use of the following lemma, whose proof can be found in [11] (Lemma 8.2).

Lemma 10.3. Suppose that $\delta_{\alpha}(x)$ is a function satisfying

$$
0 \leq \delta_{\alpha}(x) \leq C \alpha^{-3} \mathbf{1}(|x| \leq \alpha)
$$

and $\int \delta_{\alpha}(x) \mathrm{d} x=1$ (for example $\delta_{\alpha}(x)=\alpha^{-3} g(x / \alpha)$, for a bounded probability density $g(x)$ supported in $\{x:|x| \leq 1\})$. Moreover, for $J^{(k)} \in \mathcal{K}_{k}$ and for $j=$ $1, \ldots, k$, we define the norm

$$
\begin{aligned}
\left\|J^{(k)}\right\|_{j}:= & \sup _{\mathbf{x}_{k}, \mathbf{x}_{k}^{\prime}}\left\langle x_{1}\right\rangle^{4} \ldots\left\langle x_{k}\right\rangle^{4}\left\langle x_{1}^{\prime}\right\rangle^{4} \ldots\left\langle x_{k}^{\prime}\right\rangle^{4} \\
& \times\left(\left|J^{(k)}\left(\mathbf{x}_{k} ; \mathbf{x}_{k}^{\prime}\right)\right|+\left|\nabla_{x_{j}} J^{(k)}\left(\mathbf{x}_{k} ; \mathbf{x}_{k}^{\prime}\right)\right|+\left|\nabla_{x_{j}^{\prime}} J^{(k)}\left(\mathbf{x}_{k} ; \mathbf{x}_{k}^{\prime}\right)\right|\right)
\end{aligned}
$$


and $S_{j}=\left(1-\Delta_{x_{j}}\right)$ (here $\left.\langle x\rangle^{2}:=1+x^{2}\right)$. Then if $\gamma^{(k+1)}\left(\mathbf{x}_{k+1} ; \mathbf{x}_{k+1}^{\prime}\right)$ is the kernel of a density matrix on $L^{2}\left(\mathbb{R}^{3(k+1)}\right)$, we have, for any $j \leq k$,

$$
\begin{aligned}
& \mid \int \mathrm{d} \mathbf{x}_{k+1} \mathrm{~d} \mathbf{x}_{k+1}^{\prime} J^{(k)}\left(\mathbf{x}_{k} ; \mathbf{x}_{k}^{\prime}\right)\left(\delta_{\alpha_{1}}\left(x_{k+1}-x_{k+1}^{\prime}\right) \delta_{\alpha_{2}}\left(x_{j}-x_{k+1}\right)\right. \\
&-\left.\delta\left(x_{k+1}-x_{k+1}^{\prime}\right) \delta\left(x_{j}-x_{k+1}\right)\right) \gamma^{(k+1)}\left(\mathbf{x}_{k+1} ; \mathbf{x}_{k+1}^{\prime}\right) \mid \\
& \leq C_{k}\left\|J^{(k)}\right\|_{j}\left(\alpha_{1}+\sqrt{\alpha_{2}}\right) \operatorname{Tr}\left|S_{j} S_{k+1} \gamma^{(k+1)} S_{j} S_{k+1}\right| .
\end{aligned}
$$

The same bound holds if $x_{j}$ is replaced with $x_{j}^{\prime}$ in (10.21) by symmetry.

\section{Appendix A. Properties of the Cutoff function $\theta_{i}^{(n)}$}

Recall the cutoff functions $\Theta_{k}^{(n)}=\Theta_{k}^{(n)}(\mathbf{x})$ defined for $k=1, \ldots, N$ and $n \in \mathbb{N}$ in Eq. (7.4). In the following lemma, whose proof can be found in 12, Appendix A], we collect some of their important properties which were used in the energy estimate, Proposition 7.1

Lemma A.1. $\quad$ i) The functions $\Theta_{k}^{(n)}$ are monotonic in both indices; that is, for any $n, k \in \mathbb{N}$,

$$
\Theta_{k+1}^{(n)} \leq \Theta_{k}^{(n)} \leq 1, \quad \Theta_{k}^{(n+1)} \leq \Theta_{k}^{(n)} \leq 1 .
$$

Moreover, $\Theta_{k}^{(n)}$ is permutation symmetric in the first $k$ and the last $N-k$ variables.

ii) For every $k=1, \ldots, N$ and $n \in \mathbb{N}$, we have

$$
\sum_{j=1}^{N} \frac{\left|\nabla_{j} \Theta_{k}^{(n)}\right|^{2}}{\Theta_{k}^{(n)}} \leq C \ell^{-2} \Theta_{k}^{(n-1)} .
$$

iii) For every fixed $k=1, \ldots, N$ and $n \in \mathbb{N}$ we have

$$
\sum_{i, j}\left|\nabla_{i} \nabla_{j} \Theta_{k}^{(n)}\right| \leq C \ell^{-2} \Theta_{k}^{(n-1)} .
$$

Appendix B. Removal of the assumption on DeRivatives of $V$

The goal of this appendix is to explain how the assumption

$$
\left|\nabla^{\alpha} V(x)\right| \leq C \quad \text { for all } x \in \mathbb{R}^{3},|\alpha| \leq 2
$$

in Theorem 3.1 can be removed. The main observation is that (B.3) is only used in the proof of the higher order energy estimate, Proposition [7.1 in the form $\left\|\nabla V_{N}\right\|_{\infty} \leq C N^{3},\left\|\nabla^{2} V_{N}\right\|_{\infty} \leq C N^{4}$. More precisely, the estimate on $\left\|\nabla V_{N}\right\|_{\infty}$ is first used in the study of the third term on the r.h.s. of (7.19) (the third term on the r.h.s. of (7.7) in the case $k=2$ ); namely the term containing the commutator $\left[T^{1 / 2}, D_{k}\right]=\left[\left(H_{N}+N\right)^{1 / 2}, \nabla_{1} \ldots \nabla_{k}\right]$. Bounds on the first and second derivatives are also used in the proof of Lemma 7.2. However, in both cases, the final estimates turn out to be subexponentially small in $N$ (see (7.23) and (7.27)). For this reason, the proof of Proposition 7.1 remains unchanged if, instead of (B.3), we allow $V=V^{(N)}$ to depend on $N$ and only assume

$$
\left\|\nabla^{\alpha} V^{(N)}\right\|_{\infty} \leq e^{c N^{\kappa}}, \quad|\alpha| \leq 2,
$$


for some sufficiently small $\kappa>0$.

More precisely, suppose that the potential $V \geq 0$ satisfies $V(x) \leq C\langle x\rangle^{-\sigma}$ for some $\sigma>5$, with no assumptions on the derivatives $\nabla^{\alpha} V$, for $|\alpha| \geq 1$. Then consider the evolution $\psi_{N, t}=e^{-i H_{N} t} \psi_{N}$ of an initial $N$-body wave function $\psi_{N}$ satisfying the two assumptions (3.1) and (3.2), with respect to the evolution generated by the Hamiltonian

$$
H_{N}=\sum_{j=1}^{N}-\Delta_{j}+\sum_{i<j}^{N} V_{N}\left(x_{i}-x_{j}\right)
$$

with $V_{N}(x)=N^{2} V(N x)$. As in Theorem 3.1 we claim that, for every fixed $t \in \mathbb{R}$ and every $k \geq 1$, the $k$-particle marginal $\gamma_{N, t}^{(k)}$ associated with $\psi_{N, t}$ is such that

$$
\gamma_{N, t}^{(k)} \rightarrow\left|\varphi_{t}\right\rangle\left\langle\left.\varphi_{t}\right|^{\otimes k}\right.
$$

as $N \rightarrow \infty$, w.r.t. to the trace-norm topology.

To prove (B.5) we can assume, without loss of generality, that the initial data $\psi_{N}$ is such that

$$
\left\langle\psi_{N}, H_{N}^{k} \psi_{N}\right\rangle \leq C^{k} N^{k}
$$

In fact, if this is not the case, we can use the argument outlined in Section 4 (in the proof of Theorem 3.1) and based on the analysis of Section 9 (which does not use any assumption on the derivatives of $V$ ) to approximate $\psi_{N}$.

From Theorem 6.1 it follows that the sequence $\Gamma_{N, t}^{(k)}=\left\{\gamma_{N, t}^{(k)}\right\}_{k \geq 1}$ is compact with respect to the product topology $\tau_{\text {prod }}$ defined in Section 4. If we could prove, similarly to Theorem 7.3, that an arbitrary limit point $\Gamma_{\infty, t}=\left\{\gamma_{\infty, t}^{(k)}\right\}_{k \geq 1}$ satisfies the a priori estimates (7.28), it would follow from Theorem 8.1 that $\Gamma_{\infty, t}$ is a solution to the infinite hierarchy (4.4) and, by the uniqueness result of Theorem 4.1, we could conclude the proof of (B.5) using the same strategy outlined in Section 4

To prove that every limit point $\Gamma_{\infty, t}$ satisfies the a priori estimates (7.28), we introduce a potential $\widetilde{V}^{(N)}=V * \nu^{(N)}$, where $\nu^{(N)}(x)=($ const $) e^{3 N^{\kappa} / 2} \exp \left(-e^{N^{\kappa}} x^{2}\right)$ with some sufficiently small $\kappa>0$ (here the constant is chosen so that $\int \mathrm{d} x \nu^{(N)}(x)$ $=1$ ), and we consider the evolution $\widetilde{\psi}_{N, t}=e^{-i \widetilde{H}_{N} t} \psi_{N}$ of the initial data $\psi_{N}$ with respect to the modified Hamiltonian

$$
\widetilde{H}_{N}=-\sum_{j=1}^{N} \Delta_{j}+\sum_{i<j}^{N} N^{2} \widetilde{V}^{(N)}\left(N\left(x_{i}-x_{j}\right)\right) .
$$

The potential $\widetilde{V}^{(N)}$ satisfies the bounds $\left\|\nabla^{\alpha} \widetilde{V}^{(N)}\right\|_{L^{\infty}} \leq C e^{|\alpha| N^{\kappa} / 2}$ for all $|\alpha| \leq 2$. As we remarked above, this very weak control on the $L^{\infty}$ norm of $\nabla^{\alpha} \widetilde{V}^{(N)}$ is enough to prove Proposition 7.1. Therefore, it follows from Theorem 7.3 that for any fixed $t \in \mathbb{R}$ every limit point $\widetilde{\Gamma}_{\infty, t}=\left\{\widetilde{\gamma}_{\infty, t}^{(k)}\right\}_{k \geq 1}$ of the sequence $\widetilde{\Gamma}_{N, t}=\left\{\widetilde{\gamma}_{N, t}^{(k)}\right\}_{k=1}^{N}$ (w.r.t. to the product of the weak* topologies) satisfies the bound

$$
\operatorname{Tr}\left(1-\Delta_{1}\right) \ldots\left(1-\Delta_{k}\right) \widetilde{\gamma}_{\infty, t}^{(k)} \leq C^{k}
$$

for all $k \geq 1$. To show that a limit point $\Gamma_{\infty, t}=\left\{\gamma_{\infty, t}^{(k)}\right\}_{k \geq 1}$ of the original sequence $\Gamma_{N, t}=\left\{\gamma_{N, t}^{(k)}\right\}_{k \geq 1}$ also satisfies this bound, it is therefore enough to prove that, for every fixed $t \in \mathbb{R}$,

$$
\left\|\psi_{N, t}-\widetilde{\psi}_{N, t}\right\| \rightarrow 0
$$


as $N \rightarrow \infty$. In fact (B.8) immediately implies that every limit point $\gamma_{\infty, t}^{(k)}$ of $\gamma_{N, t}^{(k)}$ is also a limit point of the sequence $\widetilde{\gamma}_{N, t}^{(k)}$ and therefore satisfies (B.7).

To verify (B.8), we observe that

$$
\begin{aligned}
& \frac{\mathrm{d}}{\mathrm{d} t}\left\|\psi_{N, t}-\widetilde{\psi}_{N, t}\right\|^{2} \\
& \quad=2 \operatorname{Im}\left\langle\psi_{N, t},\left(H_{N}-\widetilde{H}_{N}\right) \widetilde{\psi}_{N, t}\right\rangle \\
& =N^{3}(N-1) \operatorname{Im}\left\langle\psi_{N, t},\left(V *\left(\delta-\nu_{N}\right)\right)\left(N\left(x_{1}-x_{2}\right)\right) \widetilde{\psi}_{N, t}\right\rangle \\
& =N^{3}(N-1) \operatorname{Im} \int \mathrm{d} \mathbf{x} \mathrm{d} y \bar{\psi}_{N, t}(\mathbf{x}) V(y)\left(\delta-\nu^{(N)}\right)\left(N\left(x_{1}-x_{2}\right)-y\right) \widetilde{\psi}_{N, t}(\mathbf{x}) \\
& =(N-1) \operatorname{Im} \int \mathrm{d} y V(y) \int \mathrm{d} \mathbf{x} \bar{\psi}_{N, t}(\mathbf{x})\left(\delta-\nu_{N}\right)\left(x_{1}-x_{2}-y / N\right) \widetilde{\psi}_{N, t}(\mathbf{x}),
\end{aligned}
$$

where we defined $\nu_{N}(x)=N^{3} \nu^{(N)}(N x)$ (this implies that $\nu_{N}(x)=\left(\right.$ const) $N^{3} e^{3 N^{\kappa} / 2}$ $\cdot e^{-N^{2} e^{N^{\kappa}} x^{2}}$ is still normalized with $\left.\left\|\nu_{N}\right\|_{1}=1\right)$. Therefore

$$
\left|\frac{\mathrm{d}}{\mathrm{d} t}\left\|\psi_{N, t}-\widetilde{\psi}_{N, t}\right\|^{2}\right| \leq C N\|V\|_{1} \sup _{y \in \mathbb{R}^{3}}\left|\left\langle\psi_{N, t},\left(\delta_{y}-\nu_{N, y}\right)\left(x_{1}-x_{2}\right) \widetilde{\psi}_{N, t}\right\rangle\right|
$$

with $\delta_{y}(x)=\delta(x-y)$ and $\nu_{N, y}(x)=\nu_{N}(x-y)$. It is simple to check that

$$
\begin{aligned}
& \sup _{y \in \mathbb{R}^{3}}\left|\left\langle\psi_{N, t},\left(\delta_{y}-\nu_{N, y}\right)\left(x_{1}-x_{2}\right) \widetilde{\psi}_{N, t}\right\rangle\right| \\
& \quad \leq C e^{-\frac{N^{\kappa}}{8}}\left\langle\psi_{N, t},\left(1-\Delta_{1}\right)\left(1-\Delta_{2}\right) \psi_{N, t}\right\rangle^{1 / 2}\left\langle\widetilde{\psi}_{N, t},\left(1-\Delta_{1}\right)\left(1-\Delta_{2}\right) \widetilde{\psi}_{N, t}\right\rangle^{1 / 2} .
\end{aligned}
$$

In fact, (B.11) can be proven using the Fourier representation

$$
\begin{array}{rl}
\left\langle\psi_{N, t},\left(\delta_{y}-\nu_{N, y}\right)\left(x_{1}-\right.\right. & \left.\left.x_{2}\right) \widetilde{\psi}_{N, t}\right\rangle \\
=\int \mathrm{d} \mathbf{p}_{N-2} \mathrm{~d} p_{1} & \mathrm{~d} p_{2} \mathrm{~d} q_{1} \mathrm{~d} q_{2} \delta\left(p_{1}+p_{2}-q_{1}-q_{2}\right) e^{i y \cdot\left(p_{1}-q_{1}\right)} \\
& \times\left(1-e^{\left.-N^{-2} e^{-N^{\kappa}}\left(p_{1}-q_{1}\right)^{2} / 4\right)}\right. \\
& \times \frac{\sqrt{\left(p_{1}^{2}+1\right)\left(p_{2}^{2}+1\right)}}{\sqrt{\left(q_{1}^{2}+1\right)\left(q_{2}^{2}+1\right)}} \widehat{\psi}_{N, t}\left(p_{1}, p_{2}, \mathbf{p}_{N-2}\right) \\
& \times \frac{\sqrt{\left(q_{1}^{2}+1\right)\left(q_{2}^{2}+1\right)}}{\sqrt{\left(p_{1}^{2}+1\right)\left(p_{2}^{2}+1\right)}} \widehat{\widetilde{\psi}}_{N, t}\left(q_{1}, q_{2}, \mathbf{p}_{N-2}\right)
\end{array}
$$


with $\mathbf{p}_{N-2}=\left(p_{3}, \ldots, p_{N}\right)$. Using the fact that $\left|1-e^{-a}\right| \underset{\approx}{\leq} C a^{1 / 4}$ for $a>0$, applying a Schwarz inequality, and changing variables $q \rightarrow p$ in $\widetilde{\psi}_{N, t}$, we obtain

$$
\begin{aligned}
& \sup _{y \in \mathbb{R}^{3}}\left|\left\langle\psi_{N, t},\left(\delta_{y}-\nu_{N, y}\right)\left(x_{1}-x_{2}\right) \widetilde{\psi}_{N, t}\right\rangle\right| \\
& \leq C e^{-\frac{N^{\kappa}}{8}} \int \mathrm{d} \mathbf{p}_{N-2} \mathrm{~d} p_{1} \mathrm{~d} p_{2} \mathrm{~d} q_{1} \mathrm{~d} q_{2} \delta\left(p_{1}+p_{2}-q_{1}-q_{2}\right) \frac{\left|q_{1}\right|^{1 / 2}+1}{\left(q_{1}^{2}+1\right)\left(q_{2}^{2}+1\right)} \\
& \times\left(p_{1}^{2}+1\right)\left(p_{2}^{2}+1\right)\left(\beta\left|\widehat{\psi}_{N, t}\left(p_{1}, p_{2}, \mathbf{p}_{N-2}\right)\right|^{2}\right. \\
&\left.+\beta^{-1}\left|\widetilde{\widetilde{\psi}}_{N, t}\left(p_{1}, p_{2}, \mathbf{p}_{N-2}\right)\right|^{2}\right)
\end{aligned}
$$

for every $\beta>0$. This implies (B.11) because

$$
\sup _{p \in \mathbb{R}^{3}} \int \mathrm{d} q \frac{|q|^{1 / 2}+1}{\left(1+q^{2}\right)\left(1+(q-p)^{2}\right)}<\infty .
$$

To bound the expectations of $\left(1-\Delta_{1}\right)\left(1-\Delta_{2}\right)$ on the r.h.s. of (B.11) we observe that, as an operator inequality on $L_{s}^{2}\left(\mathbb{R}^{3 N}\right)$, we have

$$
\begin{aligned}
\left(H_{N}+N\right)^{2} & =\left(\sum_{j=1}^{N}\left(1-\Delta_{j}\right)+\sum_{i<j} V_{N}\left(x_{i}-x_{j}\right)\right)^{2} \\
& \geq \frac{1}{2}\left(\sum_{j=1}^{N}\left(1-\Delta_{j}\right)\right)^{2}-2\left(\sum_{i<j} V_{N}\left(x_{i}-x_{j}\right)\right)^{2} \\
& \geq N(N-1)\left(1-\Delta_{1}\right)\left(1-\Delta_{2}\right)-N^{8}\|V\|_{\infty}
\end{aligned}
$$

which, by (B.6), implies that

$$
\begin{aligned}
\left\langle\psi_{N, t},\left(1-\Delta_{1}\right)\left(1-\Delta_{2}\right) \psi_{N, t}\right\rangle & \leq\left\langle\psi_{N, t},\left(\frac{\left(H_{N}+N\right)^{2}+C N^{8}}{N(N-1)}\right) \psi_{N, t}\right\rangle \\
& =\left\langle\psi_{N},\left(\frac{\left(H_{N}+N\right)^{2}+C N^{8}}{N(N-1)}\right) \psi_{N}\right\rangle \leq C N^{6} .
\end{aligned}
$$

Using (B.6) and a Schwarz inequality similar to (B.15) to compare $H_{N}^{2}$ and $\widetilde{H}_{N}^{2}$, it is simple to check that

$$
\left\langle\psi_{N}, \widetilde{H}_{N}^{2} \psi_{N}\right\rangle \leq C N^{8}
$$

and therefore, proceeding analogously to (B.15) and (B.16), we also obtain that

$$
\left\langle\widetilde{\psi}_{N, t},\left(1-\Delta_{1}\right)\left(1-\Delta_{2}\right) \widetilde{\psi}_{N, t}\right\rangle \leq C N^{6} .
$$

Inserting (B.16) and (B.17) into (B.11), and using (B.10), we find

$$
\left|\frac{\mathrm{d}}{\mathrm{d} t}\left\|\psi_{N, t}-\widetilde{\psi}_{N, t}\right\|^{2}\right| \leq C e^{-c N^{\kappa}}
$$

for some $c>0$, which implies that

$$
\left\|\psi_{N, t}-\widetilde{\psi}_{N, t}\right\| \leq C t^{1 / 2} e^{-c N^{\kappa}} \rightarrow 0
$$

as $N \rightarrow \infty$, for every fixed $t \in \mathbb{R}$. This completes the proof of $(\overline{B .8})$. 


\section{REFERENCES}

[1] Adami, R.; Bardos, C.; Golse, F.; Teta, A.: Towards a rigorous derivation of the cubic nonlinear Schrödinger equation in dimension one. Asymptot. Anal. 40 (2004), no. 2, 93-108. MR2104130(2005g:35263)

[2] Adami, R.; Golse, F.; Teta, A.: Rigorous derivation of the cubic NLS in dimension one. J. Stat. Phys. 127 (2007), no. 6, 1193-1220. MR2331036 (2008i:82055)

[3] Anderson, M.H.; Ensher, J.R.; Matthews, M.R.; Wieman, C.E.; Cornell, E.A.: Observation of Bose-Einstein condensation in a dilute atomic vapor. Science (269), 198 (1995).

[4] Bardos, C.; Golse, F.; Mauser, N.: Weak coupling limit of the $N$-particle Schrödinger equation. Methods Appl. Anal. 7 (2000), 275-293. MR1869286 (2003c:81215)

[5] Bourgain, J.: Global well-posednedd of defocusing 3D critical NLS in the radial case, Jour. Amer. Math. Soc. 12 (1999), 145-171. MR1626257 (99e:35208)

[6] Colliander, J.; Keel, M.; Staffilani, G.; Takaoka, H.; Tao, T.: Global well-posedness and scattering for the energy-critical nonlinear Schrödinger equation in $\mathbb{R}^{3}$. Ann. of Math. (2) 167 (2008), no. 3, 767-865. MR2415387

[7] Davis, K.B.; Mewes, M.-O.; Andrews, M.R.; van Druten, N.J.; Durfee, D.S.; Kurn, D.M.; Ketterle, W.: Bose-Einstein condensation in a gas of sodium atoms. Phys. Rev. Lett. (75), 3969 (1995).

[8] Elgart, A.; Erdős, L.; Schlein, B.; Yau, H.-T.: Gross-Pitaevskii equation as the mean field limit of weakly coupled bosons. Arch. Rat. Mech. Anal. 179 (2006), no. 2, 265-283. MR2209131 (2007b:81310)

[9] Elgart, A.; Schlein, B.: Mean Field Dynamics of Boson Stars. Commun. Pure Appl. Math. 60 (2007), no. 4, 500-545. MR2290709 (2009a:85001)

[10] Erdős, L.; Schlein, B.; Yau, H.-T.: Derivation of the Gross-Pitaevskii Hierarchy for the Dynamics of Bose-Einstein Condensate. Comm. Pure Appl. Math. 59 (2006), no. 12, 1659 1741. MR 2257859 (2007k:82070)

[11] Erdős, L.; Schlein, B.; Yau, H.-T.: Derivation of the cubic non-linear Schrödinger equation from quantum dynamics of many-body systems. Invent. Math. 167 (2007), 515-614. MR:2276262 (2007m:81258)

[12] Erdős, L.; Schlein, B.; Yau, H.-T.: Derivation of the Gross-Pitaevskii Equation for the Dynamics of Bose-Einstein Condensate. Preprint arXiv:math-ph/0606017. To appear in Ann. of Math.

[13] Erdős, L.; Yau, H.-T.: Derivation of the nonlinear Schrödinger equation from a many body Coulomb system. Adv. Theor. Math. Phys. 5 (2001), no. 6, 1169-1205. MR1926667 (2004c:82075)

[14] Ginibre, J.; Velo, G.: The classical field limit of scattering theory for nonrelativistic manyboson systems. I.-II. Comm. Math. Phys. 66 (1979), no. 1, 37-76, and 68 (1979), no. 1, 45-68. MR530915 (80h:81069); MR0539736 (83h:81080)

[15] Ginibre, J.; Velo, G.: On a class of nonlinear Schrödinger equations with nonlocal interactions. Math Z. 170 (1980), 109-136. MR.562582 (82c:35018)

[16] Ginibre, J.; Velo, G.: Scattering theory in the energy space for a class of nonlinear Schrödinger equations. J. Math. Pures Appl. 64 (1985), 363-401. MR839728(87i:35171)

[17] Hepp, K.: The classical limit for quantum mechanical correlation functions. Comm. Math. Phys. 35 (1974), 265-277. MR0332046 (48:10373)

[18] Lieb, E.H.; Seiringer, R.: Proof of Bose-Einstein condensation for dilute trapped gases. Phys. Rev. Lett. 88 (2002), 170409-1-4.

[19] Lieb, E.H.; Seiringer, R.; Solovej, J.P.; Yngvason, J.: The mathematics of the Bose gas and its condensation. Oberwolfach Seminars, 34. Birkhauser Verlag, Basel, 2005. MR2143817 (2006e:82001)

[20] Lieb, E.H.; Seiringer, R.; Yngvason, J.: Bosons in a trap: a rigorous derivation of the GrossPitaevskii energy functional. Phys. Rev A 61 (2000), 043602.

[21] Kato, T.: On nonlinear Schrödinger equations, Ann. Inst. H. Poincare Phys. Theor. 46 (1987), 113-129. MR877998 (88f:35133)

[22] Klainerman, S.; Machedon, M.: On the uniqueness of solutions to the Gross-Pitaevskii hierarchy. Commun. Math. Phys. 279 (2008), no. 1, 169-185. MR2377632 (2009a:35236)

[23] Michelangeli, A.: Equivalent definitions of asymptotic 100\% BEC. Il Nuovo Cimento B 123 (2008), no. 2, 181-192. 
[24] Reed, M.; Simon, B.: Methods of modern mathematical physics: Scattering Theory. Volume 3. Academic Press, 1979. MR529429 (80m:81085)

[25] Rodnianski, I.; Schlein, B.: Quantum fluctuations and rate of convergence towards mean field dynamics. Preprint arXiv:math-ph/0711.3087. To appear in Comm. Math. Phys.

[26] Rudin, W.: Functional analysis. McGraw-Hill Series in Higher Mathematics, McGraw-Hill Book Co., New York, 1973. MR0365062 (51:1315)

[27] Spohn, H.: Kinetic Equations from Hamiltonian Dynamics. Rev. Mod. Phys. 52 (1980), no. 3, 569-615. MR578142 (81e:82010)

[28] Strauss, W.: Non-linear scattering theory at low energy. J. Funct. Anal. 41 (1981), 110-133. MR614228(83b:47074a)

[29] Yajima, K.: The $W^{k, p}$-continuity of wave operators for Schrödinger operators. J. Math. Soc. Japan 47 (1995), no. 3, 551-581. MR1331331 (97f:47049)

[30] Yajima, K.: The $W^{k, p}$-continuity of wave operators for Schrödinger operators. Proc. Japan Acad. Ser. A Math. Sci. 69 (1993), no. 4, 94-98. MR1222831 (94f:47013)

Institute of Mathematics, University of Munich, Theresienstrasse 39, D-80333 MuNICH, GERMANY

DPmms, University of Cambridge, Wilberforce Road, Cambridge, CB3 0WB, United KINGDOM

Department of Mathematics, Harvard University, Cambridge, Massachusetts 02138 